\title{
Multiscale Expansions for Linear Clamped Elliptic Shells
}

\author{
ERWAN FAOU
}

\begin{abstract}
We investigate solutions of the two-dimensional Koiter model and of the three-dimensional linear shell model in the case where the shell is clamped and its mean surface is elliptic. For smooth data, these solutions admit multiscale expansions in powers of $\varepsilon^{1 / 2}$ where $\varepsilon$ denotes the (half-)thickness of the shell. Both expansions contain terms independent of $\varepsilon$ and boundary layer terms exponentially decreasing with respect to $r / \sqrt{\varepsilon}$, with $r$ the distance to the boundary of the mean surface. The expansion of the three-dimensional displacement contains supplementary boundary layers, exponentially decreasing with respect to $r / \varepsilon$ like for plates. Using these expansions we obtain sharp estimates between the two models in various norms.
\end{abstract}

\section{INTRODUCTION}

The goal of the shell theory is to find an approximation of the three-dimensional linear elastic shell problem by a two-dimensional problem posed on the mean surface. A shell is a three-dimensional object defined by a compact oriented smooth surface $S$ embedded in $\mathbb{R}^{3}$ and a thickness parameter $\varepsilon$. For $\varepsilon \leq \varepsilon_{0}$ sufficiently small, the shell is the image $\Omega^{\varepsilon}$ of the manifold $S \times(-\varepsilon, \varepsilon)$ via the application

$$
\Phi^{\varepsilon}: S \times(-\varepsilon, \varepsilon) \ni\left(P, x_{3}\right) \mapsto P+x_{3} \boldsymbol{n}(P) \in \mathbb{R}^{3},
$$

where $\boldsymbol{n}(P)$ is a unit normal vector field on $S$. Starting from the three-dimensional equations of standard linear elasticity for a homogeneous and isotropic material, different models have been derived between 1959 and 1971: see in particular KOITER [19, 20, 21], NAGHDi [25], JOHN [18], NovOzHILOV [27]. Most of the shell models rely on a $3 \times 3$ system of intrinsic equations on $S$ depending on $\varepsilon$, and write

$$
\mathrm{K}(\varepsilon):=\mathrm{M}+\varepsilon^{2} \mathrm{~B}
$$

where $\mathrm{M}$ is the membrane operator on $S$ and $\mathrm{B}$ is a bending operator. If all above authors agree with the definition of the membrane operator $M$, different expressions of $\mathrm{B}$ can be found in the literature. For general shell geometry, the most popular and natural model is the one proposed by KOITER. This model describes the displacement of the 
shell by two tensors representing the change of metric and change of curvature of the surface submitted to a displacement. Moreover this model is elliptic for $\varepsilon>0$ (see [2]). However, for $\varepsilon=0$, the nature of the membrane operator depends on the geometry of the surface. In particular, $\mathrm{M}$ is elliptic only at the points where $S$ is elliptic. The Koiter model relies partly upon computations made by JOHN in [18]. But the question of determining the best model was very controversial (see in particular the introduction in [3] and discussions in [20, 25]).

In [15] we give the expression of the most general bending operator appearing in the 3D equations, and we show how the Koiter bending operator is linked with this operator and turns to be the most natural and simple bending operator among the others. In [9] we also give a general estimate between the 3D solution and a displacement reconstructed from the Koiter model solution. The result of [15] reduces the 3D problem to a formal series $2 \mathrm{D}$ problem very similar to the Koiter model.

In the case of plates, the Koiter model splits into the membrane operator acting on the surfacic components of the displacement and the bending operator acting on the transverse displacement. On the other hand, the work in $[10,11,8]$ shows the existence of an asymptotic expansion of the 3D displacement for plates containing boundary layer terms of scale $\varepsilon$. When such an asymptotic expansion is available, we can estimate the difference between the solution of the Koiter model and the 3D solution in every norm.

In this work, we focus our attention to the special case where the mean surface $S$ of the shell is elliptic, that is when the Gaussian curvature of $S$ is strictly positive or equivalently when the principal curvatures are everywhere of the same sign. In this case, the membrane operator $M$ is elliptic (see $[16,29,4]$ ). As the bending operator $B$ is of order 4 while the membrane operator $M$ is of order 2 , the Koiter operator $K(\varepsilon)=$ $\mathrm{M}+\varepsilon^{2} \mathrm{~B}$ is a singular perturbation of the membrane operator. The framework of VISHIK \& LYUSTERNIK [30] for scalar equations can be adapted to this situation, where the equation is a system. Combining these techniques with the formal series reduction of [15] giving the structure of the 3D boundary layers, we obtain the following results:

1. We show that the 2D displacement solution of the Koiter equation admits a complete multiscale expansion including boundary layer terms of scale $\varepsilon^{1 / 2}$ using a singular perturbation theory close to [30].

2. Using the result in [15], we then show that the $3 \mathrm{D}$ displacement admits a complete multiscale expansions with $2 \mathrm{D}$ boundary layers of scale $\varepsilon^{1 / 2}$ like for the $2 \mathrm{D}$ displacement, and 3D boundary layers of scale $\varepsilon$ like for plates.

3. We use these expansions to bound the difference between the 3D displacement and $2 \mathrm{D}$ reconstructed displacements as in [20] or [4, 23]. These estimates are sharp in the sense that the error term has the same order than the first neglected term in the asymptotic.

In the following we always take as $\mathrm{K}(\varepsilon)$ the Koiter model. We now present the 3D and 2D problems, and give the main theorems. We then recall the basic results of [15] and give the plan of the paper. 


\section{A Three-dimensional PROBlem}

The boundary of the shell $\Omega^{\varepsilon}$ defined in (1.1) has three components: A lateral boundary $\Gamma_{0}^{\varepsilon}$ image of $\partial S \times(-\varepsilon, \varepsilon)$ by the application $\Phi^{\varepsilon}$, and upper and lower faces $S_{ \pm \varepsilon}$ images of $S \times\{ \pm \varepsilon\}$. We suppose that the material constituting the shell is homogeneous and isotropic, characterized by its two Lamé coefficients $\lambda$ and $\mu$. The loading forces applied to the shell are represented by a smooth vector field $f$ defined on $\Omega^{\varepsilon}$. We suppose that the shell is clamped along $\Gamma_{0}^{\varepsilon}$ and we impose the traction free condition on $S_{+\varepsilon}$ and $S_{-\varepsilon}$. The displacement of the shell is represented by the 1-form field $\boldsymbol{u}$. In Cartesian coordinates $\left\{t^{i}\right\}$ the problem then writes

$$
\left\{\begin{array}{rlll}
-\partial_{j} A^{i j k \ell} e_{k \ell}(\boldsymbol{u}) & =f^{i} & \text { in } & \Omega^{\varepsilon} \\
\mathrm{T}^{i}(\boldsymbol{u}) & =0 & \text { on } & S_{ \pm \varepsilon} \\
\boldsymbol{u} & =0 & \text { on } \Gamma_{0}^{\varepsilon}
\end{array}\right.
$$

with $A^{i j k \ell}=\lambda \delta^{i j} \delta^{k \ell}+\mu\left(\delta^{i k} \delta^{j \ell}+\delta^{i \ell} \delta^{j k}\right)$, where $\partial_{j}$ is the partial derivative with respect to $t^{i}$ and $e_{i j}(\boldsymbol{u})=\frac{1}{2}\left(\partial_{i} u_{j}+\partial_{j} u_{i}\right)$ with $\boldsymbol{u}=u_{i} \mathrm{~d} t^{i}$ in Cartesian coordinates. On the same way $f^{i}$ denote the components of the vector field in the basis $\frac{\partial}{\partial t^{i}}$. The operator $\mathrm{T}^{i}(\boldsymbol{u})$ is the natural traction operator on the faces $S_{ \pm \varepsilon}$ appearing after integration by parts in the associated bilinear form:

$$
(\boldsymbol{u}, \boldsymbol{v}) \mapsto \int_{\Omega^{\varepsilon}} A^{i j k \ell} e_{i j}(\boldsymbol{u}) e_{k \ell}(\boldsymbol{u}) \mathrm{d} t^{1} \mathrm{~d} t^{2} \mathrm{~d} t^{3}
$$

This is the classical problem of linear elasticity set in Cartesian coordinates on a shellshaped domain of $\mathbb{R}^{3}$. Korn inequality [13] implies that this problem has a unique solution in $\mathrm{H}^{1}\left(\Omega^{\varepsilon}\right)^{3}$.

On $\Omega^{\varepsilon}$, we call "normal coordinate system" a system of the form $\left(x_{\alpha}, x_{3}\right)$ induced by the mapping (1.1), where $x_{\alpha}$ is a coordinate system on $S$ and $x_{3}$ the transverse coordinate (see [15] for details). Note that the domain $\Omega^{\varepsilon}$ is foliated by the surfaces $S_{x_{3}}$ images of $S \times\left\{x_{3}\right\}$ by the diffeomorphism (1.1). In the following, we will always identify the mean surface $S_{0}$ with the abstract manifold $S$.

\section{B THE KOITER MODEL}

On the mean surface $S_{0}$, a 2D displacement is represented by the couple of a 1-form field $z_{\alpha}$ and a function $z_{3}$. We denote by $\boldsymbol{z}=\left(z_{\alpha}, z_{3}\right) \in \Gamma\left(T_{1} S_{0}\right) \times \mathscr{C}^{\infty}\left(S_{0}\right)$ such a couple. Here, $\Gamma\left(T_{1} S_{0}\right)$ denotes the space of 1-form field on $S_{0}$. As it will be of constant use, we set

$$
\Sigma\left(S_{0}\right):=\Gamma\left(T_{1} S_{0}\right) \times \mathscr{C}^{\infty}\left(S_{0}\right)
$$

the space of (smooth) 2D displacements. More generally, we denote by $\mathbf{H}^{k}\left(S_{0}\right)$ the space of 1 -forms whose both components belong to the Sobolev space $\mathrm{H}^{k}\left(S_{0}\right)$. We keep the notation $\mathrm{H}^{k}\left(S_{0}\right)$ for functions. Typical spaces for $2 \mathrm{D}$ displacements are $\mathbf{H}^{1} \times \mathrm{L}^{2}\left(S_{0}\right)$ 
and $\mathbf{H}^{1} \times \mathrm{H}^{2}\left(S_{0}\right)$. We set $a_{\alpha \beta}$ the metric tensor on $S$, and $b_{\alpha \beta}$ the curvature tensor. The Greek indices are two-dimensional varying indices. The contraction by the metric tensor yields isomorphisms between tensor spaces on $S_{0}$. We have for example $b_{\beta}^{\alpha}=a^{\alpha \sigma} b_{\sigma \beta}$.

The Koiter operator is the operator $\mathrm{K}(\varepsilon): \Sigma\left(S_{0}\right) \rightarrow \Sigma\left(S_{0}\right)$ written

$$
\mathrm{K}(\varepsilon)=\mathrm{M}+\varepsilon^{2} \mathrm{~B} .
$$

Here, $\mathrm{M}$ is the membrane operator defined by

$$
\left\{\begin{array}{l}
\mathrm{M}_{\sigma}=-\tilde{\lambda} \mathrm{D}_{\sigma} \gamma_{\nu}^{\nu}-2 \mu \mathrm{D}_{\alpha} \gamma_{\sigma}^{\alpha} \\
\mathrm{M}_{3}=-\widetilde{\lambda} b_{\alpha}^{\alpha} \gamma_{\nu}^{\nu}-2 \mu b_{\alpha}^{\beta} \gamma_{\beta}^{\alpha}
\end{array}\right.
$$

where $\lambda$ and $\mu$ are the Lamé coefficients of the material, $\widetilde{\lambda}=2 \lambda \mu(\lambda+2 \mu)^{-1}, \mathrm{D}_{\alpha}$ is the covariant derivative on $S_{0}$, and

$$
\gamma_{\alpha \beta}(\boldsymbol{z})=\frac{1}{2}\left(\mathrm{D}_{\alpha} z_{\beta}+\mathrm{D}_{\beta} z_{\alpha}\right)-b_{\alpha \beta} z_{3}
$$

is the change of metric tensor on $S_{0}$.

The operator $\mathrm{M}$ is associated with the bilinear form defined for any $\boldsymbol{z}$ and $\boldsymbol{\eta}$ in $\mathbf{H}^{1} \times \mathrm{L}^{2}\left(S_{0}\right)$ by

$$
(\boldsymbol{z}, \boldsymbol{\eta}) \mapsto a_{\mathrm{M}}(\boldsymbol{z}, \boldsymbol{\eta})=\int_{S} M^{\alpha \beta \sigma \delta} \gamma_{\alpha \beta}(\boldsymbol{z}) \gamma_{\sigma \delta}(\boldsymbol{\eta}) \mathrm{d} S_{0},
$$

where $M^{\alpha \beta \sigma \delta}=\widetilde{\lambda} a^{\alpha \beta} a^{\sigma \delta}+\mu\left(a^{\alpha \sigma} a^{\beta \delta}+a^{\alpha \delta} a^{\beta \sigma}\right)$.

The operator $\mathrm{B}$ is the bending operator defined by

$$
\left\{\begin{array}{l}
\mathrm{B}_{\sigma}=-\frac{1}{3} \widetilde{\lambda} b_{\sigma}^{\alpha} \mathrm{D}_{\alpha} \rho_{\nu}^{\nu}-\frac{1}{3} \widetilde{\lambda} \mathrm{D}_{\alpha} b_{\sigma}^{\alpha} \rho_{\nu}^{\nu}-\frac{2}{3} \mu b_{\sigma}^{\alpha} \mathrm{D}_{\nu} \rho_{\alpha}^{\nu}-\frac{2}{3} \mu \mathrm{D}_{\nu} b_{\sigma}^{\alpha} \rho_{\alpha}^{\nu}, \\
\mathrm{B}_{3}=\frac{1}{3} \tilde{\lambda} \mathrm{D}^{\alpha} \mathrm{D}_{\alpha} \rho_{\nu}^{\nu}+\frac{2}{3} \mu \mathrm{D}^{\alpha} \mathrm{D}_{\nu} \rho_{\alpha}^{\nu}-\frac{1}{3} \widetilde{\lambda} c_{\alpha}^{\alpha} \rho_{\nu}^{\nu}-\frac{2}{3} \mu c_{\alpha}^{\beta} \rho_{\beta}^{\alpha},
\end{array}\right.
$$

where $c_{\alpha}^{\beta}=b_{\alpha}^{\nu} b_{\nu}^{\beta}$ and

$$
\rho_{\alpha \beta}(\boldsymbol{z})=\mathrm{D}_{\alpha} \mathrm{D}_{\beta} z_{3}-c_{\alpha \beta} z_{3}+b_{\alpha}^{\sigma} \mathrm{D}_{\beta} z_{\sigma}+\mathrm{D}_{\alpha} b_{\beta}^{\sigma} z_{\sigma}
$$

is the change of curvature tensor. This operator is associated with the bilinear form defined for any $\boldsymbol{z}$ and $\boldsymbol{\eta}$ in $\mathbf{H}^{1} \times \mathrm{H}^{2}\left(S_{0}\right)$ by

$$
(\boldsymbol{z}, \boldsymbol{\eta}) \mapsto a_{\mathrm{B}}(\boldsymbol{z}, \boldsymbol{\eta})=\int_{S} M^{\alpha \beta \sigma \delta} \rho_{\alpha \beta}(\boldsymbol{z}) \rho_{\sigma \delta}(\boldsymbol{\eta}) \mathrm{d} S .
$$

For a given $\boldsymbol{g} \in \Sigma\left(S_{0}\right)$, we consider the solution $\boldsymbol{z} \in \Sigma\left(S_{0}\right)$ of the problem

$$
\left\{\begin{array}{l}
\mathrm{K}(\varepsilon) \boldsymbol{z}=\boldsymbol{g} \quad \text { in } \quad S_{0} \\
\left.\boldsymbol{z}\right|_{\partial S_{0}}=0 \quad \text { and }\left.\quad \partial_{r} z_{3}(\varepsilon)\right|_{\partial S_{0}}=0
\end{array}\right.
$$

The existence of $z$ is proved in [2]. 


\section{C Main Results}

We set $(r, s)$ a coordinate system in the vicinity of $\partial S_{0}$ such that $r$ is the geodesic distance to the boundary, and $s$ is the arclength along $\partial S_{0}$. We denote by $b_{s s}(r, s)$, $b_{r s}(r, s)$ and $b_{r r}(r, s)$ the components of the curvature tensor in this coordinate system. The fact that $S_{0}$ is elliptic implies that we can choose the orientation of $S_{0}$ such that $b_{s s}$ and $b_{r r}$ are positive along $S_{0}$. We denote by $\chi(r)$ a $\mathscr{C}^{\infty}$ cut-off function near $\partial S_{0}$.

To construct the expansion of the $2 \mathrm{D}$ displacement, we suppose that the right-hand side $\boldsymbol{g}=\boldsymbol{g}^{\varepsilon}$ depends on $\varepsilon$ and admits the expansion

$$
\boldsymbol{g}^{\varepsilon} \simeq \sum_{k \geq 0} \varepsilon^{k} \boldsymbol{g}^{k}
$$

where for all $k, \boldsymbol{g}^{k} \in \Sigma\left(S_{0}\right)$. This means that for any Sobolev norm on $S_{0}$ and any $N$, we have

$$
\left\|\boldsymbol{g}^{\varepsilon}-\sum_{k=0}^{N} \varepsilon^{k} \boldsymbol{g}^{k}\right\| \leq C_{N} \varepsilon^{N},
$$

where $C_{N}$ is independent on $\varepsilon$.

Theorem 1.1 Let $\boldsymbol{z}^{\varepsilon}$ be the solution of the problem (1.9) with a right-hand side $\boldsymbol{g}^{\varepsilon}$ satisfying (1.10). Then $\boldsymbol{z}^{\varepsilon}$ admits an asymptotic expansion in powers of $\varepsilon^{1 / 2}$ :

$$
\boldsymbol{z}^{\varepsilon} \simeq \sum_{k \geq 0} \varepsilon^{k / 2}\left(\boldsymbol{\zeta}^{k / 2}\left(x_{\alpha}\right)+\chi(r) \boldsymbol{Z}^{k / 2}\left(\frac{r}{\sqrt{\varepsilon}}, s\right)\right)
$$

where for all $k, \zeta^{k / 2} \in \Sigma\left(S_{0}\right)$ is independent on $\varepsilon$ and $Z^{k / 2}(T, s)$ is exponentially decreasing in $T$, uniformly in $s$ and smooth on $\mathbb{R}^{+} \times \partial S_{0}$. Moreover, for each fixed $s$, the function $T \mapsto e^{\eta T} \boldsymbol{Z}^{k / 2}(T, s)$ is bounded on $\mathbb{R}^{+}$for all $\eta<\eta_{1}$ where

$$
\eta_{1}=\left(\frac{3 \mu(\widetilde{\lambda}+\mu)}{(\widetilde{\lambda}+2 \mu)^{2}}\right)^{1 / 4} \sqrt{b_{s s}(0, s)}
$$

The first term $\zeta^{0}$ is the solution of the membrane problem

$$
\left\{\begin{aligned}
\mathbf{M} \boldsymbol{\zeta}^{0} & =\boldsymbol{g}^{0} \text { in } S_{0}, \\
\left.z_{\alpha}\right|_{\partial S_{0}} & =0,
\end{aligned}\right.
$$

where $\boldsymbol{g}^{0}$ is the first term of the asymptotic expansion of $\boldsymbol{g}^{\varepsilon}$. The fact that the membrane cannot solve for the boundary conditions on $z_{3}$ is the reason for the presence of the 2D boundary layer terms. Indeed, the third component $M_{3}$ is an operator of order 0 in $z_{3}$, while $\mathrm{B}_{3}$ is of order 4 in $z_{3}$. The first boundary layer terms satisfies $Z_{\alpha}^{0}=0$ but $Z_{3}^{0} \neq 0$ in general. 
Using the expansion (1.11) we obtain estimates between $\boldsymbol{z}^{\varepsilon}$ and $\boldsymbol{\zeta}^{0}$. For example we get

$$
\left\|\boldsymbol{z}^{\varepsilon}-\boldsymbol{\zeta}^{0}\right\|_{\mathbf{H}^{1} \times \mathrm{L}^{2}(S)} \leq C \varepsilon^{1 / 4}
$$

where $C$ is independent on $\varepsilon$. This estimate implies in particular the convergence result of [4] and improves the result in [24].

To construct the expansion of the 3D displacements we suppose that the right-hand side $\boldsymbol{f}=\boldsymbol{f}^{\varepsilon}$ depends on $\varepsilon$ in the following regular way: If $\left(x_{\alpha}, x_{3}\right)$ is a normal coordinate system on $\Omega^{\varepsilon}$ we set $X_{3}=\varepsilon^{-1} x_{3}$, and define the vector field $\boldsymbol{f}(\varepsilon)\left(x_{\alpha}, X_{3}\right)=$ $\boldsymbol{f}^{\varepsilon}\left(x_{\alpha}, x_{3}\right)$ on the manifold $\Omega:=S \times(-1,1)$. We suppose that $\boldsymbol{f}(\varepsilon)$ admits the expansion

$$
\boldsymbol{f}(\varepsilon) \simeq \sum_{k \geq 0} \varepsilon^{k} \boldsymbol{f}^{k}
$$

where for all $k, f^{k}$ is independent of $\varepsilon$ in $\Omega$. This hypothesis is satisfied in the case where $f$ is independent of $\varepsilon$ in the physical cartesian coordinates. In this case the Taylor expansion of $f$ at $x_{3}=0$ around the mid-surface yields the coefficients of the expansion (1.15).

Theorem 1.2 Let $\boldsymbol{u}^{\varepsilon}$ be the solution of (1.3) with the right-hand side $\boldsymbol{f}^{\varepsilon}$ satisfying (1.15). Then $\boldsymbol{u}^{\varepsilon}$ admits the following asymptotic expansion in powers of $\varepsilon^{1 / 2}$ :

$$
\boldsymbol{u}^{\varepsilon} \simeq \sum_{k \geq 0} \varepsilon^{k / 2}\left(\boldsymbol{v}^{k / 2}\left(x_{\alpha}, \frac{x_{3}}{\varepsilon}\right)+\chi(r) \boldsymbol{W}^{k / 2}\left(\frac{r}{\sqrt{\varepsilon}}, s, \frac{x_{3}}{\varepsilon}\right)+\chi(r) \varphi^{k / 2}\left(\frac{r}{\varepsilon}, s, \frac{x_{3}}{\varepsilon}\right)\right)
$$

where for all $k, \boldsymbol{v}^{k / 2}$ is a $\mathscr{C}^{\infty}$ 1-form field on $\Omega^{\varepsilon}$ and $\boldsymbol{W}^{k / 2}\left(T, s, X_{3}\right)$ is uniformly exponentially decreasing in $T$ with the same bound $\eta_{1}$ as in (1.12). The terms $\boldsymbol{v}^{k / 2}$ and $\boldsymbol{W}^{k / 2}$ are polynomial in $X_{3}=\varepsilon^{-1} x_{3}$ and smooth. The term $\varphi^{k / 2}\left(R, s, X_{3}\right)$ is uniformly exponentially decreasing in $R$ and has singularities near the edges of the shell.

Under the assumption (1.15), the 2D right-hand side defined by $\boldsymbol{g}^{\varepsilon}=\frac{1}{2 \varepsilon} \int_{-\varepsilon}^{\varepsilon} \boldsymbol{f}^{\varepsilon} \mathrm{d} x_{3}$ admits an expansion of the form (1.10) with $\boldsymbol{g}^{k}=\frac{1}{2} \int_{-1}^{1} \boldsymbol{f}^{k}\left(X_{3}\right) \mathrm{d} X_{3}$. In this case, the precise comparison of the first terms of $\boldsymbol{z}^{\varepsilon}$ and $\boldsymbol{u}^{\varepsilon}$ allows to write sharp estimates between the 3D displacement and the 2D Koiter and membrane models. We define $\mathrm{U}^{\mathrm{KL}} \boldsymbol{z}$ the Kirchhoff-Love displacement associated with $z$ as

$$
\mathrm{U}_{\sigma}^{\mathrm{KL}} \boldsymbol{z}=z_{\sigma}-x_{3}\left(\theta_{\sigma}(\boldsymbol{z})+b_{\sigma}^{\alpha} z_{\alpha}\right)+x_{3}^{2} b_{\sigma}^{\alpha} \theta_{\alpha}(\boldsymbol{z}) \quad \text { and } \quad \mathrm{U}_{3}^{\mathrm{KL}} \boldsymbol{z}=z_{3},
$$

where $\theta_{\alpha}(\boldsymbol{z})=\mathrm{D}_{\alpha} z_{3}+b_{\alpha}^{\beta} z_{\beta}$ and $p=\lambda(\lambda+2 \mu)^{-1}$. This displacement satisfies $e_{i 3}\left(\mathrm{U}_{\sigma}^{\mathrm{KL}} \boldsymbol{z}\right)=0$ for all $\boldsymbol{z}$.

Proposition 1.3 Suppose that $\boldsymbol{f}^{\varepsilon}$ satisfies the hypothesis (1.15), and let $\boldsymbol{u}(\varepsilon)$ be the three-dimensional displacement on the scaled domain $\Omega$. We set $\boldsymbol{g}^{\varepsilon}=\frac{1}{2 \varepsilon} \int_{-\varepsilon}^{\varepsilon} \boldsymbol{f}^{\varepsilon} \mathrm{d} x_{3}$. Let $\boldsymbol{\zeta}^{\varepsilon}$ be the solution of the membrane problem (1.13) with right-hand side $\boldsymbol{g}^{\varepsilon}$, and $\boldsymbol{z}^{\varepsilon}$ 
be the solution of the Koiter model (1.9) with the right-hand side $\boldsymbol{g}^{\varepsilon}$. Then we have the estimates

$$
\begin{array}{ll}
\left\|\boldsymbol{u}(\varepsilon)-\boldsymbol{\zeta}^{\varepsilon}\right\|_{\mathrm{H}^{1}(\Omega)^{2} \times \mathrm{L}^{2}(\Omega)} \leq C \varepsilon^{1 / 4}, & \left\|\boldsymbol{u}(\varepsilon)-\boldsymbol{z}^{\varepsilon}\right\|_{\mathrm{H}^{1}(\Omega)^{2} \times \mathrm{L}^{2}(\Omega)} \leq C \varepsilon^{1 / 4} \\
\left\|\boldsymbol{u}(\varepsilon)-\boldsymbol{z}^{\varepsilon}\right\|_{\mathrm{H}^{1}(\Omega)^{3}} \leq C \varepsilon^{1 / 4} \quad \text { and } & \left\|\boldsymbol{u}(\varepsilon)-\mathrm{U}^{\mathrm{KL}} \boldsymbol{z}^{\varepsilon}\right\|_{\mathrm{H}^{1}(\Omega)^{2} \times \mathrm{L}^{2}(\Omega)} \leq C \varepsilon^{3 / 4}
\end{array}
$$

These estimates imply the convergence results of $[5,6]$. Note that $\boldsymbol{\zeta}^{\varepsilon}$ does not converge towards $\boldsymbol{u}(\varepsilon)$ in the $\mathrm{H}^{1}(\Omega)^{3}$ norm. In the membrane norm $\mathrm{H}^{1}(\Omega)^{2} \times \mathrm{L}^{2}(\Omega)$, the convergence rate obtained with the Kirchhoff-Love displacement $U^{\mathrm{KL}} \boldsymbol{z}^{\varepsilon}$ associated with $\boldsymbol{z}^{\varepsilon}$ is the best possible using 2D objects: the leading error terms is governed by pure $3 \mathrm{D}$ effects due to the presence of boundary layer near the edges.

In energy norm, we need more terms to get an optimal estimate with the same $\boldsymbol{z}^{\varepsilon}$ : Following Koiter [20] we define the three-dimensional reconstructed displacement in normal coordinates $\mathrm{U} z$ by

$$
\mathrm{U} \boldsymbol{z}=\mathrm{U}^{\mathrm{KL}} \boldsymbol{z}+\mathrm{U}^{\mathrm{Comp}} \boldsymbol{z} \quad \text { where } \quad \mathrm{U}_{\sigma}^{\mathrm{Comp}} \boldsymbol{z}=0 \quad \text { and } \quad \mathrm{U}_{3} \boldsymbol{z}=-x_{3} p \gamma_{\alpha}^{\alpha}(\boldsymbol{z})+\frac{x_{3}^{2}}{2} p \rho_{\alpha}^{\alpha}(\boldsymbol{z}) .
$$

On the physical shell $\Omega^{\varepsilon}$, we define the energy $\mathrm{E}_{3 \mathrm{D}}^{\varepsilon}[\boldsymbol{v}]$ by the equation

$$
\mathrm{E}_{3 \mathrm{D}}^{\varepsilon}[\boldsymbol{v}]=\int_{\Omega^{\varepsilon}} A^{i j k \ell} e_{i j}(\boldsymbol{v}) e_{k \ell}(\boldsymbol{v}) \mathrm{d} V,
$$

and we write $a=\mathcal{O}\left(\varepsilon^{p}\right)$ if we have $c \varepsilon^{p} \leq a \leq C \varepsilon^{p}$ for $c$ and $C$ non zero constants independent on $\varepsilon$. With these notations, we have the result:

Theorem 1.4 Suppose that $\boldsymbol{f}^{\varepsilon}$ satisfies the hypothesis (1.15). Let $\boldsymbol{u}^{\varepsilon}$ be the solution of the three-dimensional equations (1.3) and $\boldsymbol{z}^{\varepsilon}$ be the solution of the Koiter equations (1.9) with the right-hand side $\boldsymbol{g}^{\varepsilon}=\frac{1}{2 \varepsilon} \int_{-\varepsilon}^{\varepsilon} \boldsymbol{f}^{\varepsilon} \mathrm{d} x_{3}$. Suppose that the solution of the membrane problem $\zeta^{0}$ given in (1.13) is not zero, then we have the estimates:

$$
\mathrm{E}_{3 \mathrm{D}}^{\varepsilon}\left[\boldsymbol{u}^{\varepsilon}\right]=\mathcal{O}(\varepsilon) \quad \text { and } \quad \mathrm{E}_{3 \mathrm{D}}^{\varepsilon}\left[\boldsymbol{u}^{\varepsilon}-U \boldsymbol{z}^{\varepsilon}\right] \leq C \varepsilon \mathrm{E}_{3 \mathrm{D}}^{\varepsilon}\left[\boldsymbol{u}^{\varepsilon}\right]
$$

where $C$ is independent of $\varepsilon$.

This estimates can be compared to the one initially given by KOITER in [20]. The leading error term is governed by the 3D boundary layers. It improves the result in [22] for elliptic shells.

For ease of use, the standard change of unknown $\boldsymbol{w}^{\varepsilon}=\boldsymbol{\mu}^{-1}\left(x_{3}\right) \boldsymbol{u}^{\varepsilon}$ is made (see [25]), where $\boldsymbol{\mu}\left(x_{3}\right)$ is defined by

$$
\boldsymbol{u}=\boldsymbol{\mu}\left(x_{3}\right) \boldsymbol{w}=\left\{\begin{array}{l}
u_{\alpha}=w_{\alpha}-x_{3} b_{\alpha}^{\beta} w_{\beta} \quad \text { and } \\
u_{3}=w_{3} .
\end{array}\right.
$$

Theorem 1.2 is equivalent for $\boldsymbol{u}^{\varepsilon}$ and for the shifted displacement $\boldsymbol{w}^{\varepsilon}$. 


\section{D FORMAL SERIES SOLUTION}

The proof of Theorem 1.2 is based on the results in [15] for the formal series solution of the 3D problem. We recall here this general framework.

The first step in [15] discards the lateral boundary conditions, and studies the inner $3 \mathrm{D}$ equations written in terms of the shifted displacement $\boldsymbol{w}$ :

$$
\begin{cases}\mathrm{L}\left(x_{\alpha}, x_{3} ; \mathrm{D}_{\alpha}, \partial_{3}\right) \boldsymbol{w}^{\varepsilon}=-\boldsymbol{f}^{\varepsilon} & \text { in } \Omega^{\varepsilon} \\ \mathrm{T}\left(x_{\alpha}, x_{3} ; \mathrm{D}_{\alpha}, \partial_{3}\right) \boldsymbol{w}^{\varepsilon}=0 & \text { on } S_{ \pm \varepsilon}\end{cases}
$$

where $\partial_{3}$ is the partial derivative with respect to $x_{3}$. The scaling $X_{3}=\varepsilon^{-1} x_{3}$ allows to state the problem (1.22) on the manifold $\Omega=S \times(-1,1)$ with operators $\mathrm{L}(\varepsilon)$ and $\mathrm{T}(\varepsilon)$ having the following power series expansions:

$$
\mathrm{L}(\varepsilon)=\varepsilon^{-2} \sum_{k=0}^{\infty} \varepsilon^{k} \mathrm{~L}^{k} \quad \text { and } \mathrm{T}(\varepsilon)=\varepsilon^{-1} \sum_{k=0}^{\infty} \varepsilon^{k} \mathrm{~T}^{k},
$$

with which are associated the formal series $L[\varepsilon]$ and $\mathrm{T}[\varepsilon]$ with the same coefficients (see Theorem 3.3 of [15]).

Recall that if $E$ and $F$ are two function spaces, if $a[t]=\sum_{k \geq 0} t^{k} a^{k}$ is a formal series in $t$ with coefficients $a^{k} \in \mathcal{L}(E, F)$ and $b[t]=\sum_{k \geq 0} t^{k} b^{k}$ is a formal series with coefficients $b^{k} \in E$, then the formal series $c[t]=a[t] b[t]$ is defined by the equation $c[t]=\sum_{k \geq 0} t^{k} c^{k}$ where for all $n, c^{n}=\sum_{k=0}^{n} a^{k} b^{n-k}$. This is the classical Cauchy product for formal series.

Considering the formal series $\boldsymbol{f}[\varepsilon]=\sum_{k \geq 0} \varepsilon^{k} \boldsymbol{f}^{k}$ induced by (1.15), the 3D formal series problem writes: Find a formal series $\boldsymbol{w}[\varepsilon]=\sum_{k \geq 0} \varepsilon^{k} \boldsymbol{w}^{k}$ with 1-form field coefficients, such that

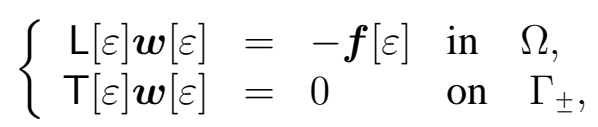

where $\Gamma_{ \pm}$are the upper and lower faces of $\Omega$.

Theorems 4.1 and 4.3 of [15] reduce this problem to a $2 \mathrm{D}$ formal series problem on $S_{0}$. There exist formal series operators $\mathrm{V}[\varepsilon], \mathrm{Q}[\varepsilon], \mathrm{A}[\varepsilon]$ and $\mathrm{G}[\varepsilon]$ such that if $\boldsymbol{z}[\varepsilon]=\sum_{k \geq 0} \varepsilon^{k} \boldsymbol{z}^{k}$ is a formal series with coefficients in $\Sigma\left(S_{0}\right)$ satisfying the equation

$$
\mathrm{A}[\varepsilon] \boldsymbol{z}[\varepsilon]=\mathrm{G}[\varepsilon] \boldsymbol{f}[\varepsilon] \text { in } S_{0},
$$

then $\boldsymbol{w}[\varepsilon]$ defined by the equation

$$
\boldsymbol{w}[\varepsilon]=\mathrm{V}[\varepsilon] \boldsymbol{z}[\varepsilon]+\mathrm{Q}[\varepsilon] \boldsymbol{f}[\varepsilon] \text { in } \Omega
$$

is solution of (1.24). The formal series $A[\varepsilon]$ writes

$$
\mathrm{A}[\varepsilon]=\mathrm{M}+\varepsilon^{2} \mathrm{~A}^{2}+\cdots,
$$


where $M$ is the membrane operator. The exact expression of $A^{2}$ is given in Theorem 4.4 of [15], and Proposition 4.5 gives an estimate of the difference between $A^{2}$ and the bending operator $B$ of the Koiter model. Moreover these operators coincide on the space of inextensional displacements (the 2D displacements $\boldsymbol{z}$ such that $\gamma_{\alpha \beta}(\boldsymbol{z})=0$ ).

In the following, we will use the fact that the formal series $\mathrm{V}[\varepsilon], \mathrm{A}[\varepsilon], \mathrm{Q}[\varepsilon]$ and $\mathrm{G}[\varepsilon]$ satisfy the functional equations

$$
\left\{\begin{array} { l } 
{ \mathrm { L } [ \varepsilon ] \mathrm { V } [ \varepsilon ] \boldsymbol { z } = - \mathcal { I } \circ \mathrm { A } [ \varepsilon ] \boldsymbol { z } , } \\
{ \mathrm { T } [ \varepsilon ] \mathrm { V } [ \varepsilon ] \boldsymbol { z } = 0 , }
\end{array} \quad \text { and } \quad \left\{\begin{array}{rl}
\mathrm{L}[\varepsilon] \mathrm{Q}[\varepsilon] \boldsymbol{f} & =\mathcal{I} \circ \mathrm{G}[\varepsilon] \boldsymbol{f}-\boldsymbol{f}, \\
\mathrm{T}[\varepsilon] \mathrm{Q}[\varepsilon] \boldsymbol{f} & =0
\end{array}\right.\right.
$$

for all $\boldsymbol{z} \in \Sigma\left(S_{0}\right)$ and $\boldsymbol{f} \in \mathscr{C}^{\infty}\left(I, \Sigma\left(S_{0}\right)\right)$. Here $\mathcal{I}$ is the canonical embedding $\mathcal{I}$ : $\Sigma\left(S_{0}\right) \mapsto \mathscr{C}^{\infty}\left(I, \Sigma\left(S_{0}\right)\right)$.

The second step in [15] (Theorem 5.3) deals with boundary layer formal series. In general, if $\boldsymbol{z}[\varepsilon]$ is a solution of (1.25), the reconstructed displacement (1.26) cannot satisfy the condition $\boldsymbol{w}[\varepsilon]=0$ on the lateral boundary. Similarly to plates (see $[26,10,8]$ ), the change of variable $R=r / \varepsilon$ allows to state the formal series problem: Find $\varphi[\varepsilon]$ with coefficients $\varphi^{k}\left(R, s, X_{3}\right)$ exponentially decreasing with respect to $R$, such that

$$
(\mathscr{L}[\varepsilon], \mathscr{T}[\varepsilon]) \boldsymbol{\varphi}[\varepsilon]=0 \quad \text { and }\left.\quad \boldsymbol{w}[\varepsilon]\right|_{\Gamma_{0}}+\left.\boldsymbol{\varphi}[\varepsilon]\right|_{R=0}=0
$$

where the formal series $\mathscr{L}[\varepsilon]$ and $\mathscr{T}[\varepsilon]$ are induced by Taylor expansions in $R=0$ and $X_{3}=0$ of the operators $\mathrm{L}$ and $\mathrm{T}$ in coordinates $\left(R, s, X_{3}\right)$, and where the formal series $\boldsymbol{w}[\varepsilon]$ is given by (1.26). Note that $R=0$ coincides with the lateral boundary $\Gamma_{0}$.

Theorem 5.3 in [15] shows that the existence of a formal series $\varphi[\varepsilon]$ solution of (1.28) relies upon compatibility conditions on $\boldsymbol{z}[\varepsilon]$ on the boundary $\partial S_{0}$. There exist formal series operators $d[\varepsilon]$ and $h[\varepsilon]$ whose coefficients define four trace operators on the boundary $\partial S_{0}$, such that if $\boldsymbol{z}[\varepsilon]$ satisfies the equation

$$
\mathrm{d}[\varepsilon] \boldsymbol{z}[\varepsilon]=\mathrm{h}[\varepsilon] \boldsymbol{f}[\varepsilon] \quad \text { on } \partial S_{0},
$$

then we can construct a formal series $\varphi[\varepsilon]$ solution of the problem (1.28). Moreover, the first term of the formal series $\mathrm{d}[\varepsilon]$ writes

$$
\mathrm{d}^{0} \boldsymbol{z}=\left.\left(z_{r}, z_{s}, z_{3}, \partial_{r} z_{3}\right)\right|_{\partial S_{0}} .
$$

This operator is the natural Dirichlet operator associated with the Koiter model $K(\varepsilon)$ for $\varepsilon>0$. As before, the formal series $\varphi[\varepsilon]$ is constructed using formal series operator satisfying functional equations of the type (1.27) in 3D boundary layer spaces (see equations (5.14) and (5.16) in [15]).

Definition 1.5 The equations

$$
\left\{\begin{aligned}
\mathrm{A}[\varepsilon] \boldsymbol{z}[\varepsilon] & =\mathrm{G}[\varepsilon] \boldsymbol{f}[\varepsilon] & & \text { in } S_{0}, \\
\mathrm{~d}[\varepsilon] \boldsymbol{z}[\varepsilon] & =\mathrm{h}[\varepsilon] \boldsymbol{f}[\varepsilon] & & \text { on } \partial S_{0},
\end{aligned}\right.
$$

define the reduced problem associated with the 3D formal series problem. 


\section{E OUtline OF The Paper}

The proof of Theorem 1.1 is given in sections 2-5. Section 2 studies the inner equations based on an inverse of the membrane operator, while Section 3 deals with the twodimensional boundary layer terms. In Section 4 we define and solve a formal series problem in powers of $\varepsilon^{1 / 2}$ and show sharp estimates in Section 5.

The proof of Theorem 1.2 is given in sections 6-8. We note that the Koiter problem (1.9) and the reduced problem (1.31) have the same first terms. In Section 6, starting from the reduced problem (1.31) posed on the mid-surface, we define a $2 \mathrm{D}$ formal series problem in powers of $\varepsilon^{1 / 2}$ including 2D boundary layers, similar to the one obtained in Section 4 for the Koiter model. The solution of this problem allows to construct the terms of the 3D expansion in Section 7. The final error estimates are given in Section 8.

The proof of Theorem 1.4 is given in Appendix B while Appendix A is devoted to a technical result needed in section 6 .

\section{KOITER MODEL INNER EQUATIONS}

We consider the solution $z \in \Sigma\left(S_{0}\right)$ of the problem

$$
\left\{\begin{array}{l}
\mathrm{K}(\varepsilon) \boldsymbol{z}=\boldsymbol{g} \quad \text { in } S_{0}, \\
\left.\boldsymbol{z}\right|_{\partial S_{0}}=\boldsymbol{c}_{*} \text { and }\left.\partial_{r} z_{3}\right|_{\partial S_{0}}=c_{n},
\end{array}\right.
$$

where $\boldsymbol{g} \in \Sigma\left(S_{0}\right), \boldsymbol{c}_{*}=\left(c_{r}, c_{s}, c_{3}\right) \in \mathscr{C}^{\infty}\left(\partial S_{0}\right)^{3}$ and $c_{n} \in \mathscr{C}^{\infty}\left(S_{0}\right)$. This is the problem (1.9) with non homogeneous boundary conditions. In the following we set $\boldsymbol{c}=$ $\left(\boldsymbol{c}_{*}, c_{3}\right) \in \mathscr{C}^{\infty}\left(\partial S_{0}\right)^{4}$. The existence of $\boldsymbol{z}$ is a consequence of the inequality

$$
\|\boldsymbol{\gamma}(\boldsymbol{z})\|_{\mathrm{L}^{2}\left(S_{0}\right)}+\|\boldsymbol{\rho}(\boldsymbol{z})\|_{\mathrm{L}^{2}\left(S_{0}\right)} \geq C\|\boldsymbol{z}\|_{\mathbf{H}^{1} \times \mathrm{H}^{2}\left(S_{0}\right)}
$$

for all $\boldsymbol{z} \in \mathbf{H}_{0}^{1} \times \mathrm{H}_{0}^{2}\left(S_{0}\right)$, see [2].

The operators $M$ and $K(\varepsilon)$ split into surfacic and transverse parts, which have the following block degrees:

$$
\operatorname{deg} M=\left(\begin{array}{ll}
2 & 1 \\
1 & 0
\end{array}\right) \quad \text { and } \quad \operatorname{deg} K(\varepsilon)=\left(\begin{array}{ll}
2 & 3 \\
3 & 4
\end{array}\right)
$$

According to [1], we say that $\mathrm{M}$ is of multidegree $(2,0)$ and $\mathrm{K}(\varepsilon)$ of multidegree $(2,4)$. The following result gives the ellipticity property of $\mathrm{M}$ in the case where $S_{0}$ is elliptic. This result can be found in [16] and [29].

Theorem 2.1 Suppose that $S_{0}$ is elliptic. The membrane operator $\mathrm{M}=\left(\mathrm{M}_{\alpha}, \mathrm{M}_{3}\right)$ acting on $\boldsymbol{z}=\left(z_{\alpha}, z_{3}\right) \in \Sigma\left(S_{0}\right)$ is strongly elliptic of multidegree $(\mathbf{2}, 0)$ in the sense of Agmon, Douglis and Nirenberg. The Dirichlet operator $\left.z \rightarrow\left(z_{r}, z_{s}\right)\right|_{\partial S_{0}}$ satisfies the 
complementing boundary condition. Moreover, the kernel of $\mathrm{M}$ with these boundary conditions is reduced to $\{0\}$, and we have for $z \in \Sigma\left(S_{0}\right)$ :

$$
\|\boldsymbol{z}\|_{\mathbf{H}^{p+1} \times \mathrm{H}^{p}\left(S_{0}\right)} \leq C\left(\|\mathbf{M} \boldsymbol{z}\|_{\mathbf{H}^{p-1} \times \mathrm{H}^{p}\left(S_{0}\right)}+\left\|\left(z_{r}, z_{s}\right)\right\|_{\mathrm{H}^{p+1 / 2}\left(\partial S_{0}\right)^{2}}\right),
$$

where $p>-\frac{1}{2}$ is a fixed regularity index and $C$ a constant depending on $S_{0}$ and $p$.

As corollary we will mainly use the following result:

Theorem 2.2 Suppose that $S_{0}$ is elliptic. Let $\boldsymbol{g} \in \Sigma\left(S_{0}\right)$ and let $c_{r}$ and $c_{s}$ two functions of $\mathscr{C}^{\infty}\left(\partial S_{0}\right)$. Then there exists a unique $\zeta \in \Sigma\left(S_{0}\right)$ such that

$$
\left\{\begin{array}{l}
\mathrm{M} \zeta=g \text { in } S_{0} \\
\left.\zeta_{r}\right|_{\partial S_{0}}=c_{r} \text { and }\left.\zeta_{s}\right|_{\partial S_{0}}=c_{s} .
\end{array}\right.
$$

Suppose that the right-hand side $\boldsymbol{g}=\boldsymbol{g}^{\varepsilon}$ in (2.1) expands in powers series of $\varepsilon$ (see (1.10)). We first seek the solution $\boldsymbol{z}^{\varepsilon}$ of (1.9) under the form $\boldsymbol{z}=\sum_{k>0} \varepsilon^{k} \boldsymbol{z}^{k}$. This yields the formal series problem

$$
\mathrm{K}[\varepsilon] \boldsymbol{z}[\varepsilon]=\boldsymbol{g}[\varepsilon]
$$

where $\mathrm{K}[\varepsilon]$ is the finite formal series $\mathrm{M}+\varepsilon^{2} \mathrm{~B}$ and $\boldsymbol{g}[\varepsilon]$ is induced by the expansion of $\boldsymbol{g}^{\varepsilon}$. This problem is equivalent to the collection of equations:

$$
\forall k \geq 0, \quad \mathbf{M} \boldsymbol{z}^{k}=-\mathrm{B} \boldsymbol{z}^{k-2}+\boldsymbol{g}^{k},
$$

where we set $\boldsymbol{z}^{k}=0$ for $k \leq 0$. The previous Theorem shows by induction the existence of solutions of these equations. However we cannot satisfy in general the whole boundary conditions $\mathrm{d}^{0} \boldsymbol{z}^{\varepsilon}=\boldsymbol{c}$ by a power series representation of the solution.

\section{TWO-DIMENSIONAL BOUNDARY LAYERS}

As the mean surface $S_{0}$ is elliptic, we have for all coordinate system and all $\left(\xi_{\alpha}\right)=$ $\left(\xi_{1}, \xi_{2}\right) \in \mathbb{R}^{2}$

$$
b^{\alpha \beta} \xi_{\alpha} \xi_{\beta} \geq c\left(\xi_{1}^{2}+\xi_{2}^{2}\right) \text { with } \quad c>0 .
$$

Using the fact that $r$ is the geodesic distance to the boundary of $S_{0}$, it is easy to show that we have $a_{r r}(r, s)=1$ and $a_{r s}(r, s)=0$ for all $(r, s)$. As $s$ is the arc-length on $\partial S_{0}$, we also have $a_{s s}(0, s)=1$ for all $s$. We thus compute that the Christoffel symbols satisfy $\Gamma_{r r}^{r}=\Gamma_{r r}^{s}=\Gamma_{r s}^{r}=0$ for all $(r, s)$. Similarly we have $\Gamma_{r s}^{s}(r, s)=$ $\frac{1}{2} a^{s s}(r, s) \partial_{r} a_{s s}(r, s), \Gamma_{s s}^{r}(r, s)=-\frac{1}{2} \partial_{r} a_{s s}(r, s)$ and $\Gamma_{s s}^{s}(r, s)=\frac{1}{2} \partial_{s} a_{s s}(r, s)$. In particular we have $\Gamma_{s s}^{s}(0, s)=0$. 
In order to construct the boundary layers, we have to match the operators $M$ and $B$. The transverse component $B_{3}$ of the bending operator $B$ is an operator of order 4 in $z_{3}$ while $M_{3}$ is an operator of order 0 in $z_{3}$. Following [30], we hence set (see also [28]):

$$
T=\varepsilon^{-1 / 2} r \quad \text { and thus } \quad \partial_{r}=\varepsilon^{-1 / 2} \partial_{T} .
$$

Setting $\mathrm{K}(\varepsilon)\left(r, s ; \partial_{r}, \partial_{s}\right)$ the operator $\mathrm{K}(\varepsilon)$ in coordinates $(r, s)$, we define the operator $\mathcal{K}(\varepsilon)$ as

$$
\mathcal{K}(\varepsilon)\left(T, s ; \partial_{T}, \partial_{s}\right):=\mathrm{K}(\varepsilon)\left(\varepsilon^{1 / 2} T, s ; \varepsilon^{-1 / 2} \partial_{T}, \partial_{s}\right) .
$$

The operator $\mathcal{K}(\varepsilon)$ acts on the manifold

$$
\breve{S}:=\left[0,+\infty\left[\times \partial S_{0} .\right.\right.
$$

Using the Taylor expansion in $T=0$ of the coefficients of the operator $\mathcal{K}(\varepsilon)$, we can associate with this operator a formal series in $\varepsilon^{1 / 2}$, written $\mathcal{K}\left[\varepsilon^{1 / 2}\right]$. As $M$ is of order 2 , we have

$$
\mathcal{K}\left[\varepsilon^{1 / 2}\right]=\sum_{k \geq-2} \varepsilon^{k / 2} \mathcal{K}^{k / 2}
$$

where $\mathcal{K}^{k / 2}: \Sigma(\breve{S}) \rightarrow \Sigma(\breve{S})$ are operators in $\partial_{T}$ and $\partial_{s}$, polynomials in $T$.

Here, as the formal series (3.4) involves powers of $\varepsilon^{1 / 2}$, it is natural to consider the general formal series problem

$$
\mathcal{K}\left[\varepsilon^{1 / 2}\right] \boldsymbol{Z}\left[\varepsilon^{1 / 2}\right]=\boldsymbol{G}\left[\varepsilon^{1 / 2}\right],
$$

where $\boldsymbol{Z}\left[\varepsilon^{1 / 2}\right]=\sum_{k \geq 0} \varepsilon^{k / 2} \boldsymbol{Z}^{k / 2}$ and $\boldsymbol{G}\left[\varepsilon^{1 / 2}\right]=\sum_{k \geq 0} \varepsilon^{k / 2} \boldsymbol{G}^{k / 2}$ are formal series with coefficients in $\Sigma(\breve{S})$.

The first non-zero term in the formal series $\mathcal{K}\left[\varepsilon^{1 / 2}\right]$ is the operator $\mathcal{K}^{-1}$ :

$$
\mathcal{K}_{T}^{-1}(\boldsymbol{Z})=-(\widetilde{\lambda}+2 \mu) \partial_{T}^{2} Z_{T}, \quad \mathcal{K}_{s}^{-1}(\boldsymbol{Z})=-\mu \partial_{T}^{2} Z_{s} \quad \text { and } \quad \mathcal{K}_{3}^{-1}(\boldsymbol{Z})=0 .
$$

In the operator $\mathcal{K}^{-1 / 2}$, the component $\mathcal{K}_{3}^{-1 / 2}$ depends only on the operator $M_{3}$ :

$$
\mathcal{K}_{3}^{-1 / 2}(\boldsymbol{Z})=-2\left(\widetilde{\lambda} H+\mu b_{r r}\right) \partial_{T} Z_{T}-2 \mu b_{r s} \partial_{T} Z_{s},
$$

where $b_{r r}, b_{r s}$ and $b_{s s}$ are the components of the second fundamental form $b_{r r}(0, s)$, $b_{r s}(0, s)$ and $b_{s s}(0, s)$ on the boundary and $H=\frac{1}{2}\left(b_{r r}+b_{s s}\right)$ is the mean curvature along $\partial S_{0}$.

The influence of the operator $B_{3}$ after the homogenization only appears in the operator $\mathcal{K}_{3}^{0}$. That is why we make a scaling in the problem (3.5) in order to obtain a formal series problem with a first operator term having all non-zero components, and taking into account the influence of the operator $B_{3}$. We set

$$
\left\{\begin{array} { l } 
{ \breve { Z } _ { \alpha } [ \varepsilon ^ { 1 / 2 } ] = Z _ { \alpha } [ \varepsilon ^ { 1 / 2 } ] , } \\
{ \breve { Z } _ { 3 } [ \varepsilon ^ { 1 / 2 } ] = \varepsilon ^ { 1 / 2 } Z _ { 3 } [ \varepsilon ^ { 1 / 2 } ] , }
\end{array} \quad \text { and } \quad \left\{\begin{array}{l}
\breve{G}_{\alpha}\left[\varepsilon^{1 / 2}\right]=\varepsilon G_{\alpha}\left[\varepsilon^{1 / 2}\right] \\
\breve{G}_{3}\left[\varepsilon^{1 / 2}\right]=\varepsilon^{1 / 2} G_{3}\left[\varepsilon^{1 / 2}\right]
\end{array}\right.\right.
$$


These relations read

$$
\forall k \geq 0, \quad\left\{\begin{array} { l } 
{ Z _ { \alpha } ^ { k / 2 } = \breve { Z } _ { \alpha } ^ { k / 2 } , } \\
{ Z _ { 3 } ^ { k / 2 } = \breve { Z } _ { 3 } ^ { ( k + 1 ) / 2 } , }
\end{array} \quad \text { and } \quad \left\{\begin{array}{l}
G_{\alpha}^{k / 2}=\breve{G}_{\alpha}^{(k+2) / 2} \\
G_{3}^{k / 2}=\breve{G}_{3}^{(k+1) / 2}
\end{array}\right.\right.
$$

If the formal series $\breve{Z}\left[\varepsilon^{1 / 2}\right]$ starts with a power 0 of $\varepsilon^{1 / 2}$, this implies that the corresponding formal series $\boldsymbol{Z}\left[\varepsilon^{1 / 2}\right]$ starts with a power $-1 / 2$ of $\varepsilon$.

We define the formal series $\breve{\mathcal{K}}\left[\varepsilon^{1 / 2}\right]$ by the formal series equation

$$
\breve{\mathcal{K}}\left[\varepsilon^{1 / 2}\right] \breve{\boldsymbol{Z}}\left[\varepsilon^{1 / 2}\right]=\left(\varepsilon \mathcal{K}_{\sigma}\left[\varepsilon^{1 / 2}\right] \boldsymbol{Z}\left[\varepsilon^{1 / 2}\right], \quad \varepsilon^{1 / 2} \mathcal{K}_{3}\left[\varepsilon^{1 / 2}\right] \boldsymbol{Z}\left[\varepsilon^{1 / 2}\right]\right) .
$$

The problem (3.5) is thus equivalent to the problem

$$
\breve{\mathcal{K}}\left[\varepsilon^{1 / 2}\right] \breve{Z}\left[\varepsilon^{1 / 2}\right]=\breve{G}\left[\varepsilon^{1 / 2}\right] .
$$

The formal series

$$
\breve{\mathcal{K}}\left[\varepsilon^{1 / 2}\right]=\sum_{k \geq 0} \varepsilon^{k / 2} \breve{\mathcal{K}}^{k / 2}
$$

has then for first term the operator $\breve{\mathcal{K}}^{0}$ whose components write, for $\breve{Z} \in \Sigma(\breve{S})$ :

$$
\begin{aligned}
\breve{\mathcal{K}}_{T}^{0}(\breve{\boldsymbol{Z}})= & -(\widetilde{\lambda}+2 \mu) \partial_{T}^{2} \breve{Z}_{T}+2\left(\widetilde{\lambda} H+\mu b_{r r}\right) \partial_{T} \breve{Z}_{3}, \\
\breve{\mathcal{K}}_{s}^{0}(\breve{\boldsymbol{Z}})= & -\mu \partial_{T}^{2} \breve{Z}_{s}+2 \mu b_{r s} \partial_{T} \breve{Z}_{3}, \\
\breve{\mathcal{K}}_{3}^{0}(\breve{\boldsymbol{Z}})= & -2\left(\widetilde{\lambda} H+\mu b_{r r}\right) \partial_{T} \breve{Z}_{T}-2 \mu b_{r s} \partial_{T} \breve{Z}_{s} \\
& +\frac{1}{3}(\widetilde{\lambda}+2 \mu) \partial_{T}^{4} \breve{Z}_{3}+4\left((\widetilde{\lambda}+2 \mu) H^{2}-\mu K\right) \breve{Z}_{3},
\end{aligned}
$$

where $K=b_{r r} b_{s s}-b_{r s}^{2}$ is the Gaussian curvature of $S_{0}$ along $\partial S_{0}$.

Note that the variable $s$ only appears in the coefficients of the operator $\breve{\mathcal{K}}^{0}$ and thus can be considered as a parameter. For fixed $s$, the operator $\breve{\mathcal{K}}^{0}$ is a system of ordinary differential equations in $T$ on the interval $[0,+\infty[$.

Proposition 3.1 Let $s \in \partial S_{0}$ fixed, and let $c_{3}, c_{n} \in \mathbb{R}$. There exists a unique function $\breve{\varphi} \in \mathscr{C}^{\infty}\left(\mathbb{R}^{+}\right)^{3}$ exponentially decreasing, solution of the system

$$
\left\{\begin{array}{l}
\breve{\mathcal{K}}^{0}(\breve{\varphi})=0 \quad \text { in } \quad[0,+\infty[ \\
\left.\breve{\varphi}_{3}\right|_{T=0}=c_{3} \text { and }\left.\partial_{T} \breve{\varphi}_{3}\right|_{T=0}=c_{n} .
\end{array}\right.
$$

Moreover, for all $\eta<\eta_{1}$ where $\eta_{1}$ is given in (1.12), $e^{\eta T} \breve{\varphi}$ is bounded as $T \rightarrow \infty$.

Proof. Let us write the symbol of the operator $\breve{\mathcal{K}}^{0}$ by replacing formally $\partial_{T}$ by $i \tau$ where $\tau$ is a complex number. This symbol writes

$$
\left(\begin{array}{ccc}
(\widetilde{\lambda}+2 \mu) \tau^{2} & 0 & 2 i\left(\widetilde{\lambda} H+\mu b_{r r}\right) \tau \\
0 & \mu \tau^{2} & 2 i \mu b_{r s} \tau \\
-2 i\left(\widetilde{\lambda} H+\mu b_{r r}\right) \tau & -2 i \mu b_{r s} \tau & \frac{1}{3}(\widetilde{\lambda}+2 \mu) \tau^{4}+b
\end{array}\right),
$$


where $b=4\left((\widetilde{\lambda}+2 \mu) H^{2}-\mu K\right)$. The determinant of this matrix is

$$
\mu \tau^{4}\left(\frac{1}{3}(\widetilde{\lambda}+2 \mu)^{2} \tau^{4}+4 \mu(\tilde{\lambda}+\mu) b_{s s}^{2}\right) .
$$

This polynomial in $\tau$ has 0 as root of order 4 , and 4 complex roots

$$
e^{\frac{i \pi}{4}} \sqrt[4]{a}, e^{-\frac{i \pi}{4}} \sqrt[4]{a}, e^{\frac{3 i \pi}{4}} \sqrt[4]{a} \quad \text { and } \quad e^{-\frac{3 i \pi}{4}} \sqrt[4]{a}, \quad \text { where } \quad a=\frac{12 \mu(\widetilde{\lambda}+\mu)}{(\widetilde{\lambda}+2 \mu)^{2}} b_{s s}^{2}>0 .
$$

Among these roots, only two have positive imaginary parts: $e^{\frac{i \pi}{4}} \sqrt[4]{a}$ and $e^{\frac{3 i \pi}{4}} \sqrt[4]{a}$. These roots are

$$
\eta_{1}(1+i), \quad \eta_{1}(1-i), \quad-\eta_{1}(1+i) \quad \text { and } \quad-\eta_{1}(1-i) .
$$

For fixed $s$, consider now the equation $\breve{\mathcal{K}}^{0}(\breve{\varphi})=0$ in $\mathbb{R}^{+}$. As $\left(\widetilde{\lambda} H+\mu b_{r r}\right) \neq 0$, we can transform this system in a triangular system written

$$
\begin{aligned}
2\left(\widetilde{\lambda} H+\mu b_{r r}\right) \partial_{T} \breve{\varphi}_{T}+2 \mu b_{r s} \partial_{T} \breve{\varphi}_{s} & \\
-\frac{1}{3}(\widetilde{\lambda}+2 \mu) \partial_{T}^{4} \breve{\varphi}_{3}-4\left((\widetilde{\lambda}+2 \mu) H^{2}-\mu K\right) \breve{\varphi}_{3} & =0 \\
\mu \partial_{T}^{2} \breve{\varphi}_{s}-2 \mu b_{r s} \partial_{T} \breve{\varphi}_{3} & =0 \\
\left(\frac{1}{3}(\widetilde{\lambda}+2 \mu)^{2} \partial_{T}^{4}+4 \mu(\widetilde{\lambda}+\mu) b_{s s}^{2}\right) \partial_{T} \breve{\varphi}_{3} & =0 .
\end{aligned}
$$

We deduce from the last equation that $\breve{\varphi}_{3}$ writes

$$
\breve{\varphi}_{3}=A_{1}+A_{2} e^{-T \eta_{1}(1+i)}+A_{3} e^{-T \eta_{1}(1-i)}+A_{4} e^{T \eta_{1}(1+i)}+A_{5} e^{T \eta_{1}(1+i)},
$$

where $A_{1}, A_{2}, A_{3}, A_{4}$ and $A_{5}$ are complex numbers. As we seek $\breve{\varphi}$ exponentially decreasing in $T$, we deduce that $A_{1}=A_{4}=A_{5}=0$. Using the boundary conditions in (3.13) we deduce that

$$
\breve{\varphi}_{3}(T)=e^{-\eta_{1} T}\left(\frac{1}{\eta_{1}} c_{n} \sin \left(\eta_{1} T\right)+c_{3}\left(\cos \left(\eta_{1} T\right)+\sin \left(\eta_{1} T\right)\right)\right) .
$$

The second equation in (3.16) then shows that

$$
\breve{\varphi}_{s}(T)=2 b_{r s} \int_{T}^{+\infty} \breve{\varphi}_{3}(T) \mathrm{d} T
$$

is the unique solution $\breve{\varphi}_{s}$ exponentially decreasing. Finally the equation

$$
\begin{aligned}
& \breve{\varphi}_{T}(T)=-\mu b_{r s}\left(\widetilde{\lambda} H+\mu b_{r r}\right)^{-1} \breve{\varphi}_{s}(T) \\
& \quad+\left(\widetilde{\lambda} H+\mu b_{r r}\right)^{-1} \int_{T}^{\infty}\left(\frac{1}{6}(\widetilde{\lambda}+2 \mu) \partial_{T}^{4} \breve{\varphi}_{3}(T)+2\left((\widetilde{\lambda}+2 \mu) H^{2}-\mu K\right) \breve{\varphi}_{3}(T)\right) \mathrm{d} T
\end{aligned}
$$

yields the unique solution exponentially decreasing of the first equation in (3.16).

In order to solve the system (3.13) with non-zero right-hand sides, we define the space

$$
\mathfrak{T}\left(\mathbb{R}^{+}\right):=\left\{f \in \mathscr{C}^{\infty}\left(\mathbb{R}^{+}\right) \quad \mid \quad \forall i, k \in \mathbb{N}, \quad \forall \eta<\eta_{1}, \quad e^{\eta T} \partial_{T}^{i} f \in \mathrm{L}^{2}\left(\mathbb{R}^{+}\right)\right\} .
$$


To this function space we associate the displacement space

$$
\mathfrak{T}\left(\mathbb{R}^{+}\right):=\left\{\breve{\varphi}=\left(\breve{\varphi}_{T}, \breve{\varphi}_{s}, \breve{\varphi}_{3}\right) \in \mathfrak{T}\left(\mathbb{R}^{+}\right)^{3}\right\} .
$$

If $\breve{G} \in \mathfrak{T}\left(\mathbb{R}^{+}\right)$, we can construct on the interval $[0,+\infty$ [ a solution exponentially decreasing of the system

$$
\left\{\begin{array}{l}
\breve{\mathcal{K}}^{0}(\breve{\boldsymbol{Z}})=\breve{\boldsymbol{G}} \quad \text { in } \quad[0,+\infty[ \\
\breve{Z}_{3}=\partial_{T} \breve{Z}_{3}=0 \quad \text { for } \quad T=0
\end{array}\right.
$$

using explicit integral representation: see the formulae page 48 in [1]. This particular solution is then exponentially decreasing with an exponent smaller than $\eta_{1}$. Hence we have the following result:

Theorem 3.2 Let $s \in \partial S_{0}$ fixed. Let $\breve{G} \in \mathfrak{T}\left(\mathbb{R}^{+}\right)$, and let $c_{3}, c_{n} \in \mathbb{R}$. There exists a unique $\breve{Z} \in \mathfrak{T}\left(\mathbb{R}^{+}\right)$solution of the system

$$
\left\{\begin{array}{l}
\breve{\mathcal{K}}^{0}(\breve{\boldsymbol{Z}})=\breve{\boldsymbol{G}} \text { in } \quad \mathbb{R}^{+}, \\
\left.\breve{Z}_{3}\right|_{T=0}=c_{3} \quad \text { and }\left.\quad \partial_{T} \breve{Z}_{3}\right|_{T=0}=c_{n}
\end{array}\right.
$$

Moreover, if the right-hand side $\breve{G} \in \mathscr{C}^{\infty}\left(\partial S_{0}, \mathfrak{T}\left(\mathbb{R}^{+}\right)\right)$and if $c_{3}(s)$ and $c_{n}(s)$ are $\mathscr{C}^{\infty}$ functions on $\partial S_{0}$, then the solution $\breve{Z}$ of $(3.22)$ defines an element of $\mathscr{C}^{\infty}\left(\partial S_{0}, \mathfrak{T}\left(\mathbb{R}^{+}\right)\right)$.

\section{CONSTRUCTION OF THE KOITER MODEL EXPANSION}

\section{A FORMAL SERIES PROBLEM}

The operator $\mathrm{K}(\varepsilon)$ induces in a natural way a formal series in $\varepsilon^{1 / 2}$ by setting

$$
\mathrm{K}\left[\varepsilon^{1 / 2}\right]:=\mathrm{M}+\varepsilon^{2} \mathrm{~B}=\sum_{k \geq 0} \varepsilon^{k / 2} \mathrm{~K}^{k / 2},
$$

with $\mathrm{K}^{0}=\mathrm{M}, \mathrm{K}^{2}=\mathrm{B}$ and $\mathrm{K}^{k / 2}=0$ for $k=1,2,3$ and $k \geq 5$. Hence if $\boldsymbol{\zeta}\left[\varepsilon^{1 / 2}\right]=\sum_{k \geq 0} \varepsilon^{k / 2} \boldsymbol{\zeta}^{k / 2}$ and $\boldsymbol{g}\left[\varepsilon^{1 / 2}\right]=\sum_{k \geq 0} \varepsilon^{k / 2} \boldsymbol{g}^{k / 2}$ are formal series with coefficients in $\Sigma\left(S_{0}\right)$, the equation

$$
\mathrm{K}\left[\varepsilon^{1 / 2}\right] \boldsymbol{\zeta}\left[\varepsilon^{1 / 2}\right]=\boldsymbol{g}\left[\varepsilon^{1 / 2}\right]
$$

makes sense.

Let $\boldsymbol{g}\left[\varepsilon^{1 / 2}\right]$ and $\boldsymbol{G}\left[\varepsilon^{1 / 2}\right]$ be formal series with coefficients in the spaces $\Sigma\left(S_{0}\right)$ and $\mathscr{C}^{\infty}\left(\partial S_{0}, \mathfrak{T}\left(\mathbb{R}^{+}\right)\right)$respectively. The equations (4.1) and (3.5) yield the equations to be satisfied by $\zeta\left[\varepsilon^{1 / 2}\right]$ and $Z\left[\varepsilon^{1 / 2}\right]$ in the interior of the domains. The goal is now to match these formal series along the boundary. 
On the boundary $\partial S_{0}$, which correspond to the set $T=0$ in $\breve{S}$, we can define the following formal series, with coefficients in $\mathscr{C}^{\infty}\left(\partial S_{0}\right)$ :

$$
\left.\boldsymbol{\zeta}\left[\varepsilon^{1 / 2}\right]\right|_{\partial S_{0}}:=\left.\sum_{k \geq 0} \varepsilon^{k / 2} \boldsymbol{\zeta}^{k / 2}\right|_{\partial S_{0}} \quad \text { and }\left.\quad \boldsymbol{Z}\left[\varepsilon^{1 / 2}\right]\right|_{\partial S_{0}}:=\left.\sum_{k \geq 0} \varepsilon^{k / 2} \boldsymbol{Z}^{k / 2}\right|_{T=0} .
$$

Moreover, we can define the reentrant normal derivative of the formal series $\zeta\left[\varepsilon^{1 / 2}\right]$ in the vicinity of $\partial S$ by the formula

$$
\partial_{r} \boldsymbol{\zeta}\left[\varepsilon^{1 / 2}\right]:=\sum_{k \geq 0} \varepsilon^{k / 2} \partial_{r} \boldsymbol{\zeta}^{k / 2}
$$

But the relation $T=\varepsilon^{-1 / 2} r$ allows to define the action of $\partial_{r}$ on the formal series $Z\left[\varepsilon^{1 / 2}\right]$ by the formula

$$
\partial_{r} \boldsymbol{Z}\left[\varepsilon^{1 / 2}\right]:=\varepsilon^{-1 / 2} \partial_{T} \boldsymbol{Z}\left[\varepsilon^{1 / 2}\right]=\sum_{k \geq-1} \varepsilon^{k / 2} \partial_{T} \boldsymbol{Z}^{(k+1) / 2}(T, s) .
$$

If we set $\zeta_{3}^{-1 / 2}=0$, we thus can consider the sum

$$
\left.\partial_{r} \zeta_{3}\left[\varepsilon^{1 / 2}\right]\right|_{\partial S_{0}}+\left.\partial_{r} Z_{3}\left[\varepsilon^{1 / 2}\right]\right|_{T=0}=\sum_{k \geq-1} \varepsilon^{k / 2}\left(\left.\partial_{r} \zeta_{3}^{k / 2}\right|_{\partial S_{0}}+\left.\partial_{T} Z_{3}^{(k+1) / 2}\right|_{T=0}\right) .
$$

Suppose given formal series in $\varepsilon^{1 / 2}$

$$
\boldsymbol{c}_{*}\left[\varepsilon^{1 / 2}\right]=\sum_{k \geq 0} \varepsilon^{k / 2} \boldsymbol{c}_{*}^{k / 2} \quad \text { and } \quad c_{n}\left[\varepsilon^{1 / 2}\right]=\sum_{k \geq 0} \varepsilon^{k / 2} c_{n}^{k / 2},
$$

where for all $k, \boldsymbol{c}_{*}^{k / 2}=\left(c_{r}^{k / 2}, c_{s}^{k / 2}, c_{3}^{k / 2}\right) \in \mathscr{C}^{\infty}\left(\partial S_{0}\right)^{3}$, and $c_{n}^{k / 2} \in \mathscr{C}^{\infty}\left(\partial S_{0}\right)$. We write $\boldsymbol{c}\left[\varepsilon^{1 / 2}\right]=\sum_{k \geq 0} \varepsilon^{k / 2} \boldsymbol{c}^{k / 2}$ with $\boldsymbol{c}^{k / 2}=\left(\boldsymbol{c}_{*}^{k / 2}, c_{n}^{k / 2}\right)$.

The formal series problem states as follows: Find $\left(\zeta\left[\varepsilon^{1 / 2}\right], Z\left[\varepsilon^{1 / 2}\right]\right)$ solution of the equations

$$
\left\{\begin{aligned}
\mathrm{K}\left[\varepsilon^{1 / 2}\right] \boldsymbol{\zeta}\left[\varepsilon^{1 / 2}\right] & =\boldsymbol{g}\left[\varepsilon^{1 / 2}\right], \\
\mathcal{K}\left[\varepsilon^{1 / 2}\right] \boldsymbol{Z}\left[\varepsilon^{1 / 2}\right] & =\boldsymbol{G}\left[\varepsilon^{1 / 2}\right], \\
\left.\boldsymbol{\zeta}\left[\varepsilon^{1 / 2}\right]\right|_{\partial S_{0}}+\left.\boldsymbol{Z}\left[\varepsilon^{1 / 2}\right]\right|_{T=0} & =\boldsymbol{c}_{*}\left[\varepsilon^{1 / 2}\right], \\
\left.\partial_{r} \zeta_{3}\left[\varepsilon^{1 / 2}\right]\right|_{\partial S_{0}}+\left.\partial_{r} Z_{3}\left[\varepsilon^{1 / 2}\right]\right|_{T=0} & =c_{n}\left[\varepsilon^{1 / 2}\right] .
\end{aligned}\right.
$$

Using the previous equations and the relations (3.9), the system (4.4) is equivalent to the following problem: Find formal series $\boldsymbol{\zeta}\left[\varepsilon^{1 / 2}\right]=\sum_{k \geq 0} \varepsilon^{k / 2} \boldsymbol{\zeta}^{k / 2}$ and $\breve{\boldsymbol{Z}}\left[\varepsilon^{1 / 2}\right]=$ $\sum_{k \geq 0} \varepsilon^{k / 2} \breve{Z}^{k / 2}$ solutions of the following equations, for all $k \geq 0$,

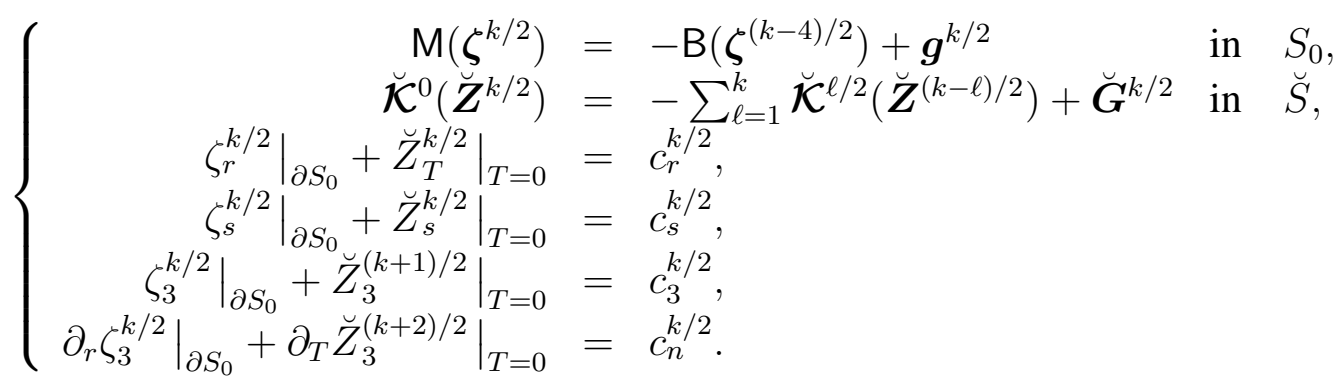


In the following, we always set the terms with negative indices to zero. We will see now how the properties of the operators $M$ and $\breve{\mathcal{K}}^{0}$ yield a solution of the system (4.5).

\section{B EXISTENCE THEOREM}

The goal of this section is to prove the following result:

Theorem 4.1 Suppose that $S_{0}$ is elliptic. Let $\boldsymbol{g}\left[\varepsilon^{1 / 2}\right]=\sum_{k \geq 0} \varepsilon^{k / 2} \boldsymbol{g}^{k / 2}$ a formal series with coefficients in $\Sigma\left(S_{0}\right), \boldsymbol{G}\left[\varepsilon^{1 / 2}\right]=\sum_{k \geq 0} \varepsilon^{k / 2} \boldsymbol{G}^{k / 2}$ a formal series with coefficients in $\mathscr{C}^{\infty}\left(\partial S_{0}, \mathfrak{T}\left(\mathbb{R}^{+}\right)\right)$, and $\boldsymbol{c}\left[\varepsilon^{1 / 2}\right]=\sum_{k \geq 0} \varepsilon^{k / 2} \boldsymbol{c}^{k / 2}$ a formal series with coefficients in $\mathscr{C}^{\infty}\left(\partial S_{0}\right)^{4}$. Then there exists a unique couple $\left(\boldsymbol{\zeta}\left[\varepsilon^{1 / 2}\right], \boldsymbol{Z}\left[\varepsilon^{1 / 2}\right]\right)$ of formal series with coefficients in $\Sigma\left(S_{0}\right)$ and $\mathscr{C}^{\infty}\left(\partial S_{0}, \mathfrak{T}\left(\mathbb{R}^{+}\right)\right)$respectively, solution of the system (4.4).

Proof. We show the result by induction. The relations (3.9) show that the first terms of the formal series $\breve{G}\left[\varepsilon^{1 / 2}\right]$ write

$$
\breve{G}^{0}=0 \quad \text { and } \quad \breve{G}^{1 / 2}=\left(0, G_{3}^{0}\right) .
$$

We divide the equations (4.5) into two parts:

$$
\forall k \geq 0,\left\{\begin{aligned}
\breve{\mathcal{K}}^{0}\left(\breve{\boldsymbol{Z}}^{k / 2}\right) & =-\sum_{\ell=1}^{k} \breve{\mathcal{K}}^{\ell / 2}\left(\breve{\boldsymbol{Z}}^{(k-\ell) / 2}\right)+\breve{\boldsymbol{G}}^{k / 2} \text { in } \breve{S}, \\
\left.\breve{Z}_{3}^{k / 2}\right|_{T=0} & =c_{3}^{(k-1) / 2}-\left.\zeta_{3}^{(k-1) / 2}\right|_{\partial S_{0}}, \\
\left.\partial_{T} \breve{Z}_{3}^{k / 2}\right|_{T=0} & =c_{n}^{(k-2) / 2}-\left.\partial_{r} \zeta_{3}^{(k-2) / 2}\right|_{\partial S_{0}},
\end{aligned}\right.
$$

for the boundary layer terms, and

$$
\forall k \geq 0,\left\{\begin{array}{l}
\mathrm{M}\left(\boldsymbol{\zeta}^{k / 2}\right)=-\mathrm{B}\left(\boldsymbol{\zeta}^{(k-4) / 2}\right)+\boldsymbol{g}^{k / 2} \text { in } S_{0}, \\
\left.\zeta_{r}^{k / 2}\right|_{\partial S_{0}}=c_{r}^{k / 2}-\left.\breve{Z}_{T}^{k / 2}\right|_{T=0}, \\
\left.\zeta_{s}^{k / 2}\right|_{\partial S_{0}}=c_{s}^{k / 2}-\left.\breve{Z}_{s}^{k / 2}\right|_{T=0},
\end{array}\right.
$$

for the terms in $\Sigma\left(S_{0}\right)$. Note that these two groups of equations are linked by terms on the boundary $\partial S_{0}$.

For $k=0$, these equations write

$$
\left\{\begin{array} { r l } 
{ \breve { \mathcal { K } } ^ { 0 } ( \breve { \boldsymbol { Z } } ^ { 0 } ) } & { = 0 } \\
{ \breve { Z } _ { 3 } ^ { 0 } | _ { T = 0 } } & { = 0 , } \\
{ \breve { Z } _ { 3 } ^ { 0 } | _ { T = 0 } } & { = 0 }
\end{array} \quad \text { in } \quad \breve { S } , \quad \text { and } \quad \left\{\begin{array}{rl}
\mathrm{M}\left(\boldsymbol{\zeta}^{0}\right) & =\boldsymbol{g}^{0} \text { in } S_{0}, \\
\left.\zeta_{r}^{0}\right|_{\partial S_{0}} & =c_{r}^{0}-\left.\breve{Z}_{T}^{0}\right|_{T=0} \\
\left.\zeta_{s}^{0}\right|_{\partial S_{0}} & =c_{s}^{0}-\left.\breve{Z}_{s}^{0}\right|_{T=0}
\end{array}\right.\right.
$$

Using Theorem 3.2, the first group of equations implies that $\breve{Z}^{0}=0$. Theorem 2.2 shows that there exists $\zeta^{0} \in \Sigma\left(S_{0}\right)$ solution of the second group. 
For $k=1$, the equations (4.6) and (4.7) write, using $\breve{Z}^{0}=0$

$$
\left\{\begin{aligned}
\breve{\mathcal{K}}^{0}\left(\breve{\boldsymbol{Z}}^{1 / 2}\right) & =\breve{G}^{1 / 2} \quad \text { in } \quad \breve{S}, \\
\left.\breve{Z}_{3}^{1 / 2}\right|_{T=0} & =c_{3}^{0}-\left.\zeta_{3}^{0}\right|_{\partial S}, \quad \text { and } \quad\left\{\left.\begin{array}{rl}
\mathrm{M}\left(\boldsymbol{\zeta}^{1 / 2}\right) & =\boldsymbol{g}^{1 / 2} \text { in } S_{0}, \\
\left.\zeta_{r}^{1 / 2}\right|_{\partial S_{0}} & =c_{r}^{1 / 2}-\left.\breve{Z}_{T}^{1 / 2}\right|_{T=0} \\
\left.\partial_{T} \breve{Z}_{3}^{1 / 2}\right|_{T=0} ^{1 / 2} & =0,
\end{array}\right|_{\partial S_{0}}=c_{s}^{1 / 2}-\left.\breve{Z}_{s}^{1 / 2}\right|_{T=0}\right.
\end{aligned}\right.
$$

Theorem 3.2 shows the existence of $\breve{Z}^{1 / 2} \in \mathscr{C}^{\infty}\left(\partial S_{0}, \mathfrak{T}\left(\mathbb{R}^{+}\right)\right)$satisfying the first group of equations (the term $\zeta^{0}$ being determined), and Theorem 2.2 shows the existence of $\zeta^{1 / 2} \in \Sigma\left(S_{0}\right)$ satisfying the second group of equations.

Let us suppose that $\boldsymbol{\zeta}^{\ell / 2} \in \Sigma(S)$ and $\breve{Z}^{\ell / 2} \in \mathscr{C}^{\infty}\left(\partial S_{0}, \mathfrak{T}\left(\mathbb{R}^{+}\right)\right)$are determined for $\ell=0, \ldots, k-1$ where $k$ is an integer, such that the equations (4.6) and (4.7) are satisfied up to the order $k$. Thanks to the structure of the operators $\breve{\mathcal{K}}^{\ell / 2}$ for $\ell \geq 0$ and using the definition of the space $\mathfrak{T}\left(\mathbb{R}^{+}\right)$, we see that the right-hand side of the inner equation of (4.6) is an element of $\mathscr{C}^{\infty}\left(\partial S_{0}, \mathfrak{T}\left(\mathbb{R}^{+}\right)\right)$. Theorem 3.2 then shows the existence of $\breve{Z}^{k / 2}$ in the space $\mathscr{C}^{\infty}\left(\partial S_{0}, \mathfrak{T}\left(\mathbb{R}^{+}\right)\right)$.

Theorem 2.2 then shows the existence of a solution $\zeta^{k / 2} \in \Sigma\left(S_{0}\right)$ of the equations (4.7) This shows the induction hypothesis at the rank $k$ and concludes the proof.

In the previous proof, the fact that $\breve{Z}^{0}=0$ implies using (3.9) that $Z_{3}^{-1 / 2}=0$ and $Z_{\alpha}^{0}=0$. The first boundary layer term is hence $Z^{0}=\left(0, Z_{3}^{0}\right)$. Thus we have

$$
\boldsymbol{\zeta}\left[\varepsilon^{1 / 2}\right]=\boldsymbol{\zeta}^{0}+\sum_{k \geq 1} \varepsilon^{k / 2} \boldsymbol{\zeta}^{k / 2} \quad \text { and } \quad \boldsymbol{Z}\left[\varepsilon^{1 / 2}\right]=\left(0, Z_{3}^{0}\right)+\sum_{k \geq 1} \varepsilon^{k / 2} \boldsymbol{Z}^{k / 2} .
$$

\section{C INFLUENCE OF THE RIGHT-HAND SIDES}

We study now 6 generic cases mentioned in Table 1 for the formal series right-hand sides in the problem (4.4). For certain cases we can show that some first terms vanish and the structure of the formal series $\left(\zeta\left[\varepsilon^{1 / 2}\right], Z\left[\varepsilon^{1 / 2}\right]\right)$ is not the same as (4.10).

Using the linearity of the problem, we only consider right-hand sides formal series of the form $\boldsymbol{g}\left[\varepsilon^{1 / 2}\right]=\boldsymbol{g}^{0}, \boldsymbol{G}\left[\varepsilon^{1 / 2}\right]=\boldsymbol{G}^{0}$ and $\boldsymbol{c}\left[\varepsilon^{1 / 2}\right]=\boldsymbol{c}^{0}=\left(c_{r}^{0}, c_{s}^{0}, c_{3}^{0}, c_{n}^{0}\right)$ and we study successively the cases where only one component does not vanish in these formal series.

Note that in all cases, the proof of Theorem 4.1 shows that $\breve{Z}^{0}$ is zero. We summarize the results by the following table. The first three columns give the expression of the righthand sides formal series, and the last two give the expression of the first terms in the expansions of $\zeta\left[\varepsilon^{1 / 2}\right]$ and $\boldsymbol{Z}\left[\varepsilon^{1 / 2}\right]$ of the solution. 


\begin{tabular}{|c|c|c||c|c|}
\hline $\boldsymbol{g}\left[\varepsilon^{1 / 2}\right]$ & $\boldsymbol{G}\left[\varepsilon^{1 / 2}\right]$ & $\boldsymbol{c}\left[\varepsilon^{1 / 2}\right]$ & $\boldsymbol{Z}\left[\varepsilon^{1 / 2}\right]$ & $\boldsymbol{\zeta}\left[\varepsilon^{1 / 2}\right]$ \\
\hline $\boldsymbol{g}^{0}$ & 0 & 0 & $\left(0, Z_{3}^{0}\right)+\cdots$ & $\boldsymbol{\zeta}^{0}+\cdots$ \\
\hline 0 & $\left(G_{\alpha}^{0}, 0\right)$ & 0 & $\left(0, \varepsilon^{1 / 2} Z_{3}^{1 / 2}\right)+\cdots$ & $\varepsilon \boldsymbol{\zeta}^{1}+\cdots$ \\
\hline 0 & $\left(0, G_{3}^{0}\right)$ & 0 & $\left(0, Z_{3}^{0}\right)+\cdots$ & $\varepsilon^{1 / 2} \boldsymbol{\zeta}^{1 / 2}+\cdots$ \\
\hline 0 & 0 & $\left(c_{\alpha}^{0}, 0,0\right)$ & $\left(0, Z_{3}^{0}\right)+\cdots$ & $\boldsymbol{\zeta}^{0}+\cdots$ \\
\hline 0 & 0 & $\left(0, c_{3}^{0}, 0\right)$ & $\left(0, Z_{3}^{0}\right)+\cdots$ & $\varepsilon^{1 / 2} \boldsymbol{\zeta}^{1 / 2}+\cdots$ \\
\hline 0 & 0 & $\left(0,0, c_{n}^{0}\right)$ & $\left(0, \varepsilon^{1 / 2} Z_{3}^{1 / 2}\right)+\cdots$ & $\varepsilon \boldsymbol{\zeta}^{1}+\cdots$ \\
\hline
\end{tabular}

Table 1. Structure of the solution with respect to the right-hand sides.

For example in the case where $\boldsymbol{g}^{0}=0, \boldsymbol{G}^{0}=\left(G_{\alpha}^{0}, 0\right)$ and $\boldsymbol{c}^{0}=0$, we have $\breve{G}^{0}=\breve{G}^{1 / 2}=0$. Studying successively the equations (4.8) and (4.9), we easily see that $\zeta^{0}=0, \breve{Z}^{1 / 2}=0$ and $\zeta^{1 / 2}=0$. In general we have $\breve{Z}^{1} \neq 0$ because this latter term satisfies

$$
\breve{\mathcal{K}}^{0}\left(\breve{\boldsymbol{Z}}^{1}\right)=\breve{\boldsymbol{G}}^{1}=\left(G_{\alpha}^{0}, 0\right) \quad \text { in } \quad \breve{S} \quad \text { and }\left.\quad \breve{Z}_{3}^{1}\right|_{\partial S}=\left.\partial_{T} \breve{Z}_{3}^{1}\right|_{\partial S}=0
$$

This implies that in general $\zeta^{1} \neq 0$. Thus the expansion is of the form

$$
\boldsymbol{\zeta}\left[\varepsilon^{1 / 2}\right]=\varepsilon \boldsymbol{\zeta}^{1}+\sum_{k \geq 3} \varepsilon^{k / 2} \boldsymbol{\zeta}^{k / 2} \quad \text { and } \quad \boldsymbol{Z}\left[\varepsilon^{1 / 2}\right]=\left(0, \varepsilon^{1 / 2} Z_{3}^{0}\right)+\sum_{k \geq 2} \varepsilon^{k / 2} \boldsymbol{Z}^{k / 2} .
$$

The other cases are studied similarly (see [14] for details).

\section{ESTIMATES FOR THE KOITER MODEL ASYMPTOTICS}

We consider the solution $\boldsymbol{z}^{\varepsilon}$ of the equations (1.9) in the case where $\boldsymbol{g}=\boldsymbol{g}^{\varepsilon}$ satisfies (1.10). Using the results of the previous section, there exist two formal series $\zeta\left[\varepsilon^{1 / 2}\right]$ and $\boldsymbol{Z}\left[\varepsilon^{1 / 2}\right]$ satisfying the equations (4.4) with formal series right-hand sides $\boldsymbol{c}\left[\varepsilon^{1 / 2}\right]=0$, $\boldsymbol{G}\left[\varepsilon^{1 / 2}\right]=0$ and $\boldsymbol{g}\left[\varepsilon^{1 / 2}\right]$ the formal series induced by the expansion (1.10).

Note that for $k \geq 0$, the sum $\boldsymbol{\zeta}^{k / 2}+\boldsymbol{Z}^{k / 2}$ does not make sense, because the terms $\boldsymbol{\zeta}^{k / 2}$ and $\boldsymbol{Z}^{k / 2}$ are not of the same nature and lives on different manifolds. However the sum $\boldsymbol{\zeta}^{k / 2}+\chi(r) \boldsymbol{Z}^{k / 2}$ makes sense and defines an element of $\Sigma\left(S_{0}\right)$. In this section we prove that the expansion

$$
\sum_{k \geq 0} \varepsilon^{k / 2}\left(\boldsymbol{\zeta}^{k / 2}+\chi(r) \boldsymbol{Z}^{k / 2}\right)
$$


is an asymptotic expansion of the solution $\boldsymbol{z}^{\varepsilon}$ of the problem. Notice that we could also make the more general assumption

$$
\boldsymbol{g}^{\varepsilon} \simeq \sum_{k \geq 0} \varepsilon^{k / 2}\left(\boldsymbol{g}^{k / 2}+\chi(r) \boldsymbol{G}^{k / 2}\right)
$$

and similarly the data on the boundary may expand in powers of $\varepsilon^{1 / 2}$.

For $N \in \mathbb{N}$ we define the 2D displacement

$$
\boldsymbol{z}^{N}(\varepsilon):=\sum_{k=0}^{N} \varepsilon^{k / 2}\left(\boldsymbol{\zeta}^{k / 2}+\chi(r) \boldsymbol{Z}^{k / 2}\right) \in \Sigma\left(S_{0}\right) .
$$

We thus have $\boldsymbol{z}^{N}(\varepsilon)=\boldsymbol{\theta}^{N}(\varepsilon)+\chi(r) \boldsymbol{\Lambda}^{N}(\varepsilon)$ with

$$
\boldsymbol{\theta}^{N}(\varepsilon):=\sum_{k=0}^{N} \varepsilon^{k / 2} \boldsymbol{\zeta}^{k / 2} \quad \text { and } \quad \boldsymbol{\Lambda}^{N}(\varepsilon):=\sum_{k=0}^{N} \varepsilon^{k / 2} \boldsymbol{Z}^{k / 2} .
$$

Using the estimate (2.2) we see that we have

$$
\langle\mathrm{K}(\varepsilon) \boldsymbol{\eta}, \boldsymbol{\eta}\rangle_{\mathrm{L}^{2}\left(S_{0}\right)^{3}} \geq C \varepsilon^{2}\|\boldsymbol{\eta}\|_{\mathbf{H}^{1} \times \mathrm{H}^{2}\left(S_{0}\right)}^{2}
$$

for all $\boldsymbol{\eta} \in \Sigma\left(S_{0}\right)$ satisfying the boundary conditions $\left.\boldsymbol{\eta}\right|_{\partial S_{0}}=0$ and $\left.\partial_{r} \eta_{3}\right|_{\partial S_{0}}=0$. In the following $C$ denotes always a constant independent of $\varepsilon$. Using this estimate we show the following result:

Proposition 5.1 For all $N \in \mathbb{N}$, we have the estimate

$$
\left\|\boldsymbol{z}^{\varepsilon}-\boldsymbol{z}^{N}(\varepsilon)\right\|_{\mathbf{H}^{1} \times \mathrm{H}^{2}\left(S_{0}\right)} \leq C \varepsilon^{N / 2-9 / 4} .
$$

Proof. Using the proof of Theorem 4.1, we see that the term $\boldsymbol{z}^{N}(\varepsilon)$ satisfies

$$
\left.\boldsymbol{z}^{N}(\varepsilon)\right|_{\partial S_{0}}=0 \quad \text { et }\left.\quad \partial_{r} z_{3}^{N}(\varepsilon)\right|_{\partial S_{0}}=\left.\varepsilon^{N / 2} \partial_{r} \boldsymbol{\zeta}_{3}^{N / 2}\right|_{\partial S_{0}} .
$$

We thus define the following element of $\Sigma\left(S_{0}\right)$ :

$$
\boldsymbol{t}^{N}:=\left(\begin{array}{c}
0 \\
-\left(\left.\partial_{r} \boldsymbol{\zeta}_{3}^{N / 2}\right|_{\partial S_{0}}\right) r \chi(r)
\end{array}\right) .
$$

It is clear that the term $\overline{\boldsymbol{z}}^{N}(\varepsilon):=\boldsymbol{z}^{N}(\varepsilon)+\varepsilon^{N / 2} \boldsymbol{t}^{N}$ satisfies the homogeneous Dirichlet boundary conditions $\left.\overline{\boldsymbol{z}}^{N}(\varepsilon)\right|_{\partial S_{0}}=0$ and $\left.\partial_{r} \bar{z}_{3}^{N}(\varepsilon)\right|_{\partial S_{0}}=0$. We thus can apply estimate (5.3) to the term $\boldsymbol{z}^{\varepsilon}-\overline{\boldsymbol{z}}^{N}(\varepsilon)$ and we have to estimate the term $\mathrm{K}(\varepsilon)\left(\boldsymbol{z}^{\varepsilon}-\overline{\boldsymbol{z}}^{N}(\varepsilon)\right)$.

The formal series $\boldsymbol{\zeta}\left[\varepsilon^{1 / 2}\right]$ satisfies the equation $\mathrm{K}\left[\varepsilon^{1 / 2}\right] \boldsymbol{\zeta}\left[\varepsilon^{1 / 2}\right]=\boldsymbol{g}\left[\varepsilon^{1 / 2}\right]$. Thus for $N \in$ $\mathbb{N}$ we have

$$
\mathrm{K}(\varepsilon) \boldsymbol{\theta}^{N}(\varepsilon)-\boldsymbol{g}^{\varepsilon}=\mathcal{O}\left(\varepsilon^{(N+1) / 2}\right),
$$


where $\mathcal{O}\left(\varepsilon^{(N+1) / 2}\right)$ denotes an element of $\Sigma\left(S_{0}\right)$ bounded in any functional norm on $S_{0}$ by $C_{N} \varepsilon^{(N+1) / 2}$ where $C_{N}$ is independent of $\varepsilon$. We deduce that we have

$$
\mathrm{K}(\varepsilon)\left(\boldsymbol{\theta}^{N}(\varepsilon)+\varepsilon^{N / 2} \boldsymbol{t}^{N}\right)-\boldsymbol{g}^{\varepsilon}=\mathcal{O}\left(\varepsilon^{N / 2}\right) .
$$

Moreover, for $\boldsymbol{y} \in \Sigma\left(S_{0}\right)$ satisfying the homogeneous boundary conditions, we have the relation

$$
\left|\left\langle\mathrm{K}(\varepsilon)\left(\chi \boldsymbol{\Lambda}^{N}(\varepsilon)\right), \boldsymbol{y}\right\rangle_{\mathrm{L}^{2}\left(S_{0}\right)^{3}}-\left\langle\mathrm{K}(\varepsilon)\left(\boldsymbol{\Lambda}^{N}(\varepsilon)\right), \chi \boldsymbol{y}\right\rangle_{\mathrm{L}^{2}\left(S_{0}\right)^{3}}\right| \leq C e^{-\beta / \sqrt{\varepsilon}}\|\boldsymbol{y}\|_{\mathbf{H}^{1} \times \mathrm{H}^{2}\left(S_{0}\right)},
$$

where $\eta_{1}>\beta>0$. This relation is due to the fact that the support of $\partial_{r} \chi(r)$ lies in an open set $\left(\rho_{1}, \rho_{2}\right) \times \partial S_{0}$ of $S_{0}$ with $0<\rho_{1}<\rho_{2}$. As this domain is at a distance of $\partial S_{0}$ independent of $\varepsilon$ and as the terms $Z\left(\frac{r}{\sqrt{\varepsilon}}, s\right)$ are uniformly exponentially decreasing with respect to $T=\frac{r}{\sqrt{\varepsilon}}$, we get the exponential term in (5.6).

Using the scaling (3.8) and the definition (3.2), we have in coordinates $(T, s)$ for all $\boldsymbol{y}$ satisfying the homogeneous boundary conditions,

$$
\left\langle\mathrm{K}(\varepsilon)\left(\boldsymbol{\Lambda}^{N}(\varepsilon)\right), \chi \boldsymbol{y}\right\rangle_{L^{2}\left(S_{0}\right)^{3}}=\frac{1}{\sqrt{\varepsilon}}\left\langle\breve{\mathcal{K}}(\varepsilon)\left(\breve{\boldsymbol{\Lambda}}^{N}(\varepsilon)\right), \breve{\boldsymbol{y}}\right\rangle_{\mathrm{L}^{2}(\breve{S})^{3}},
$$

where we set

$$
\breve{\boldsymbol{y}}(T, s):=\chi\left(\varepsilon^{1 / 2} T\right)\left(y_{T}, y_{s}, \varepsilon^{1 / 2} y_{3}\right)\left(\varepsilon^{1 / 2} T, s\right),
$$

and where

$$
\breve{\Lambda}^{N}(\varepsilon)=\sum_{k=0}^{N} \varepsilon^{k / 2} \breve{Z}^{k / 2}+\varepsilon^{(N+1) / 2}\left(0, \breve{Z}_{3}^{(N+1) / 2}\right) .
$$

Note that the scalar product in (5.7) makes sense since $\breve{y}$ has compact support.

It is clear that the Taylor expansion in $T=0$ of the operator $\breve{\mathcal{K}}(\varepsilon)$ corresponds to the formal series $\breve{\mathcal{K}}\left[\varepsilon^{1 / 2}\right]$. As the formal series $\boldsymbol{Z}\left[\varepsilon^{1 / 2}\right]$ satisfies the equation $\breve{\mathcal{K}}\left[\varepsilon^{1 / 2}\right] \breve{\boldsymbol{Z}}\left[\varepsilon^{1 / 2}\right]=$ 0 . We thus deduce that

$$
\left\langle\mathrm{K}(\varepsilon)\left(\boldsymbol{\Lambda}^{N}(\varepsilon)\right), \chi \boldsymbol{y}\right\rangle_{\mathrm{L}^{2}\left(S_{0}\right)^{3}}=\mathcal{O}\left(\varepsilon^{N / 2}\right)\|\breve{\boldsymbol{y}}\|_{\mathbf{H}^{1} \times \mathrm{H}^{2}(\breve{S})} .
$$

By doing the change of coordinates, we easily see that

$$
\|\breve{\boldsymbol{y}}\|_{\mathbf{H}^{1} \times \mathrm{H}^{2}(\breve{S})} \leq C \varepsilon^{-1 / 4}\|\boldsymbol{y}\|_{\mathbf{H}^{1} \times \mathrm{H}^{2}\left(S_{0}\right)} .
$$

Finally, we have

$$
\left\langle\mathrm{K}(\varepsilon)\left(\boldsymbol{\Lambda}^{N}(\varepsilon)\right), \chi \boldsymbol{y}\right\rangle_{\mathrm{L}^{2}\left(S_{0}\right)^{3}}=\mathcal{O}\left(\varepsilon^{N / 2-1 / 4}\right)\|\boldsymbol{y}\|_{\mathbf{H}^{1} \times \mathrm{H}^{2}\left(S_{0}\right)} .
$$

Grouping together the equations (5.5), (5.6) and (5.8), we obtain that for $\boldsymbol{y} \in \Sigma\left(S_{0}\right)$, we have

$$
\left\langle\mathrm{K}(\varepsilon)\left(\boldsymbol{z}^{\varepsilon}-\overline{\boldsymbol{z}}^{N}(\varepsilon)\right), \boldsymbol{y}\right\rangle_{\mathrm{L}^{2}\left(S_{0}\right)^{3}}=\mathcal{O}\left(\varepsilon^{N / 2-1 / 4}\right)\|\boldsymbol{y}\|_{\mathbf{H}^{1} \times \mathrm{H}^{2}\left(S_{0}\right)} .
$$

As $\left.\left(\boldsymbol{z}^{\varepsilon}-\overline{\boldsymbol{z}}^{N}(\varepsilon)\right)\right|_{\partial S_{0}}=0$ and $\left.\partial_{r}\left(\boldsymbol{z}^{\varepsilon}-\overline{\boldsymbol{z}}^{N}(\varepsilon)\right)\right|_{\partial S_{0}}=0$, we can apply this estimate to 
$\boldsymbol{y}=\boldsymbol{z}^{\varepsilon}-\overline{\boldsymbol{z}}^{N}(\varepsilon)$. Using (5.3) we obtain

$$
\left\|\boldsymbol{z}^{\varepsilon}-\overline{\boldsymbol{z}}^{N}(\varepsilon)\right\|_{\mathbf{H}^{1} \times \mathrm{H}^{2}\left(S_{0}\right)} \leq C \varepsilon^{N / 2-9 / 4} .
$$

But we have $\left\|\overline{\boldsymbol{z}}^{N}(\varepsilon)-\boldsymbol{z}^{N}(\varepsilon)\right\|_{\mathbf{H}^{1} \times \mathrm{H}^{2}\left(S_{0}\right)}=\mathcal{O}\left(\varepsilon^{N / 2}\right)$, and hence the previous estimate shows the proposition.

This proposition gives a rough estimate for the difference $\boldsymbol{z}^{\varepsilon}-\boldsymbol{z}^{N}(\varepsilon)$. We deduce now the following result:

Theorem 5.2 Let $\boldsymbol{z}^{\varepsilon}$ be the solution of (1.9) with a right-hand side satisfying (1.10), and let $\boldsymbol{z}^{N}(\varepsilon)$ defined by (5.2). For all $N \in \mathbb{N}$, we have the estimates:

$$
\left\|\boldsymbol{z}^{\varepsilon}-\boldsymbol{z}^{N}(\varepsilon)\right\|_{\mathbf{H}^{1} \times \mathrm{H}^{2}\left(S_{0}\right)} \leq C \varepsilon^{N / 2-1 / 4} \quad \text { and } \quad\left\|\boldsymbol{z}^{\varepsilon}-\boldsymbol{z}^{N}(\varepsilon)\right\|_{\mathbf{H}^{1} \times \mathrm{L}^{2}\left(S_{0}\right)} \leq C \varepsilon^{N / 2+1 / 4}
$$

This Theorem implies Theorem 1.1.

Proof. The estimate (5.4) shows that fo $N \in \mathbb{N}$, we have

$$
\left\|\boldsymbol{z}^{\varepsilon}-\boldsymbol{z}^{N+6}(\varepsilon)\right\|_{\mathbf{H}^{1} \times \mathrm{H}^{2}\left(S_{0}\right)} \leq C \varepsilon^{N / 2+3 / 4} .
$$

Thus we have

$$
\begin{aligned}
& \left\|\boldsymbol{z}^{\varepsilon}-\boldsymbol{z}^{N}(\varepsilon)\right\|_{\mathbf{H}^{1} \times \mathrm{H}^{2}\left(S_{0}\right)} \\
& \quad \leq \sum_{k=N+1}^{N+6} \varepsilon^{k / 2}\left(\left\|\boldsymbol{\zeta}^{k / 2}\right\|_{\mathbf{H}^{1} \times \mathrm{H}^{2}\left(S_{0}\right)}+\left\|\chi \boldsymbol{Z}^{k / 2}\right\|_{\mathbf{H}^{1} \times \mathrm{H}^{2}\left(S_{0}\right)}\right)+C \varepsilon^{N / 2+3 / 4} .
\end{aligned}
$$

For $k \geq 0$, the terms $\left\|\boldsymbol{\zeta}^{k / 2}\right\|_{\mathbf{H}^{1} \times \mathrm{H}^{2}\left(S_{0}\right)}$ are bounded by constants independent of $\varepsilon$. Moreover we see that for a fixed component $i$ and for all $k \geq 0$, we have the estimates $\left\|\chi Z_{i}^{k / 2}\right\|_{\mathrm{L}^{2}\left(S_{0}\right)} \leq C \varepsilon^{1 / 4}, \quad\left\|\chi Z_{i}^{k / 2}\right\|_{\mathrm{H}^{1}\left(S_{0}\right)} \leq C \varepsilon^{-1 / 4} \quad$ and $\quad\left\|\chi Z_{i}^{k / 2}\right\|_{\mathrm{H}^{2}\left(S_{0}\right)} \leq C \varepsilon^{-3 / 4}$.

Plugging these estimates into (5.10), we get

$$
\left\|\boldsymbol{z}^{\varepsilon}-\boldsymbol{z}^{N}(\varepsilon)\right\|_{\mathbf{H}^{1} \times \mathrm{H}^{2}\left(S_{0}\right)} \leq C_{N} \varepsilon^{N / 2-1 / 4},
$$

where $C_{N}$ is a constant independent of $\varepsilon$. This shows the result.

Similarly we obtain inequalities of the form (5.10) for the norm $\mathbf{H}^{1} \times \mathrm{L}^{2}\left(S_{0}\right)$ and conclude using (5.11).

Using the previous Theorem, we can compare $\boldsymbol{z}^{\varepsilon}$ with the solution of the membrane problem.

Proposition 5.3 Let $\boldsymbol{g} \in \Sigma\left(S_{0}\right)$ independent of $\varepsilon$, and let $\boldsymbol{\zeta} \in \Sigma\left(S_{0}\right)$ and $\boldsymbol{z}^{\varepsilon} \in \Sigma\left(S_{0}\right)$ 
be the solutions of the problems

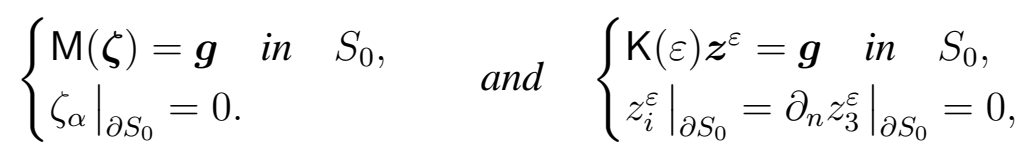

then we have the estimate

$$
\left\|\boldsymbol{z}^{\varepsilon}-\boldsymbol{\zeta}\right\|_{\mathbf{H}^{1} \times \mathrm{L}^{2}\left(S_{0}\right)} \leq C \varepsilon^{1 / 4}
$$

Proof. The previous Theorem for $N=0$ and the $\mathbf{H}^{1} \times \mathrm{L}^{2}\left(S_{0}\right)$ norm yields

$$
\left\|\boldsymbol{z}^{\varepsilon}-\boldsymbol{\zeta}^{0}-\chi \boldsymbol{Z}^{0}\right\|_{\mathbf{H}^{1} \times \mathrm{L}^{2}\left(S_{0}\right)} \leq C \varepsilon^{1 / 4} .
$$

As $\zeta=\zeta^{0}$ we thus find

$$
\left\|\boldsymbol{z}^{\varepsilon}-\boldsymbol{\zeta}\right\|_{\mathbf{H}^{1} \times \mathrm{L}^{2}\left(S_{0}\right)} \leq\left\|\chi Z_{3}^{0}\right\|_{\mathrm{L}^{2}\left(S_{0}\right)}+C \varepsilon^{1 / 4}
$$

and the equation (5.11) shows the result.

\section{FORMAL SERIES SOLUTION OF THE REDUCED PROBLEM}

We study now the problem (1.31). Note that this problem is close to the Koiter equations (1.9). In particular, the first term $\left(A^{0}, d^{0}\right)$ in (1.31) is equal to the first term $\left(K^{0}, d^{0}\right)$ of the Koiter operator and is non invertible. As before, we introduce $2 \mathrm{D}$ boundary layers to solve the problem.

\section{A STATEMENT OF THE FORMAL SERIES EQUATIONS}

In the following, we denote by $f\left[\varepsilon^{1 / 2}\right]$ the formal series induced by the expansion (1.15). Notice that every formal series in powers of $\varepsilon$ is also a formal series in powers of $\varepsilon^{1 / 2}$. We denote for example by $A\left[\varepsilon^{1 / 2}\right]$ the formal series $A[\varepsilon]$ viewed as a formal series in $\varepsilon^{1 / 2}$, and we use similar notations for the formal series of the paragraph 1.D.

(i) INNER EQUATIONS. Let $\boldsymbol{\zeta}\left[\varepsilon^{1 / 2}\right]=\sum_{k>0} \varepsilon^{k / 2} \boldsymbol{\zeta}^{k / 2}$ be a formal series with coefficients in $\Sigma\left(S_{0}\right)$. We consider the equation (see (1.31))

$$
\mathrm{A}\left[\varepsilon^{1 / 2}\right] \boldsymbol{\zeta}\left[\varepsilon^{1 / 2}\right]=\mathrm{G}\left[\varepsilon^{1 / 2}\right] \boldsymbol{f}\left[\varepsilon^{1 / 2}\right]
$$

which means

$$
\forall n \geq 0, \quad \sum_{\ell=0}^{n} \mathrm{~A}^{\ell / 2} \boldsymbol{\zeta}^{(n-\ell) / 2}=\sum_{\ell=0}^{n} \mathrm{G}^{\ell / 2} \boldsymbol{f}^{(n-\ell) / 2},
$$

where $\mathrm{A}^{\ell / 2}=0$ when $\ell$ is odd. 
In order to define the equations acting on $2 \mathrm{D}$ boundary layer terms, we make the change of variable $(r, s) \mapsto(T, s)$ with $T=\varepsilon^{-1 / 2} r$ in the formal series $\mathrm{A}\left[\varepsilon^{1 / 2}\right]$. In coordinates $(T, s)$, the operators $\mathrm{A}^{k}$ change to operators depending on $\varepsilon$ in the variable $(T, s)$. We define $\mathcal{A}^{(k)}(\varepsilon)$ the operator acting on $\breve{S}$ by the equation

$$
\forall k \geq 0, \quad \mathcal{A}^{(k)}(\varepsilon)\left(T, s ; \partial_{T}, \partial_{s}\right):=\mathrm{A}^{k}\left(\varepsilon^{1 / 2} T, s ; \varepsilon^{-1 / 2} \partial_{T}, \partial_{s}\right) .
$$

Note that these operators contains negative powers of $\varepsilon^{1 / 2}$, depending on the degree of $\mathrm{A}^{k}$. The Taylor expansions of the coefficients of these operators in $T=0$ yield formal series $\mathcal{A}^{(k)}\left[\varepsilon^{1 / 2}\right]$ with operator coefficients in variables $(T, s)$. We then have the following result:

Lemma 6.1 The Taylor expansions in $T=0$ of the coefficients of the operators $\mathcal{A}^{(k)}(\varepsilon)$ defines operators $\mathcal{A}^{k, \ell / 2}$ acting on $\breve{S}$, polynomials in $T$, such that

$$
\forall k \geq 0 \quad \mathcal{A}^{(k)}\left[\varepsilon^{1 / 2}\right]=\sum_{\ell \geq-(k+2)} \varepsilon^{\ell / 2} \mathcal{A}^{k, \ell / 2} .
$$

The equation

$$
\mathcal{A}\left[\varepsilon^{1 / 2}\right]:=\sum_{k \geq 0} \varepsilon^{k} \mathcal{A}^{(k)}\left[\varepsilon^{1 / 2}\right]
$$

then defines a formal series

$$
\mathcal{A}\left[\varepsilon^{1 / 2}\right]:=\sum_{n \geq-2} \varepsilon^{n / 2} \mathcal{A}^{n / 2} \quad \text { with } \quad \mathcal{A}^{n / 2}=\sum_{k=0}^{n+2} \mathcal{A}^{k,(n-2 k) / 2} .
$$

Proof. The equation (6.2) is a consequence of the estimate of the derivative orders of the operators $A^{k}$ (see equation (4.25) of [15]): The components of the operators $A^{k}$ are at most of degree $k+2$.

The fact that (6.3) indeed defines a formal series is then easily seen by identifying the powers of $\varepsilon^{1 / 2}$ in (6.3).

The formal series $\mathcal{A}\left[\varepsilon^{1 / 2}\right]$ corresponds to the the formal series $\mathcal{K}\left[\varepsilon^{1 / 2}\right]$ for the Koiter model. We thus consider the problem: Find a formal series $Z\left[\varepsilon^{1 / 2}\right]$ with coefficients in the space $\mathscr{C}^{\infty}\left(\partial S_{0}, \mathfrak{T}\left(\mathbb{R}^{+}\right)\right)$such that

$$
\mathcal{A}\left[\varepsilon^{1 / 2}\right] \boldsymbol{Z}\left[\varepsilon^{1 / 2}\right]=0 .
$$

As for the Koiter model, we make a change of unknown in order to obtain a new formal series problem with a formal series operator having all its first components non zero: We define the formal series $\breve{\mathcal{A}}\left[\varepsilon^{1 / 2}\right]$ by the equation (see (3.10))

$$
\breve{\mathcal{A}}\left[\varepsilon^{1 / 2}\right] \breve{\boldsymbol{Z}}\left[\varepsilon^{1 / 2}\right]=\left(\varepsilon \mathcal{A}_{\sigma}\left[\varepsilon^{1 / 2}\right] \boldsymbol{Z}\left[\varepsilon^{1 / 2}\right], \varepsilon^{1 / 2} \mathcal{A}_{3}\left[\varepsilon^{1 / 2}\right] \boldsymbol{Z}\left[\varepsilon^{1 / 2}\right]\right)
$$


where $\breve{Z}\left[\varepsilon^{1 / 2}\right]$ and $Z\left[\varepsilon^{1 / 2}\right]$ are linked by (3.8). The problem (6.5) is hence equivalent to the problem

$$
\breve{\mathcal{A}}\left[\varepsilon^{1 / 2}\right] \breve{Z}\left[\varepsilon^{1 / 2}\right]=0 .
$$

Now we have:

Proposition 6.2 The operator $\breve{\mathcal{A}}^{0}$ is the same as the operator $\breve{\mathcal{K}}^{0}$ defined in (3.12). In particular the operator $\breve{\mathcal{A}}^{0}$ satisfies Theorem 3.2.

Proof. The first terms of the formal series $\mathcal{A}[\varepsilon]$ write

$$
\mathcal{A}[\varepsilon]=\varepsilon^{-1} \mathcal{A}^{-1}+\varepsilon^{-1 / 2} \mathcal{A}^{-1 / 2}+\mathcal{A}^{0}+\cdots,
$$

with, using the fact that $A^{1}=0$,

$$
\mathcal{A}^{-1}=\mathcal{A}^{0,-1}, \quad \mathcal{A}^{-1 / 2}=\mathcal{A}^{0,-1 / 2} \quad \text { and } \quad \mathcal{A}^{0}=\mathcal{A}^{0,0}+\mathcal{A}^{2,-2} .
$$

As $\mathrm{A}^{0}$ is the membrane operator, we have that $\mathcal{A}^{0,-1}=\mathcal{K}^{-1}$ given in (3.6). Similarly, the transverse component $\mathcal{A}_{3}^{0,-1 / 2}$ is the same as $\mathcal{K}_{3}^{-1 / 2}$ given by (3.7).

Moreover, from the expression of $\mathrm{A}^{2}$ (see Equation (4.32) of [15]) we have

$$
\mathcal{A}_{3}^{2,-2}(\boldsymbol{Z})=\frac{1}{3}(\widetilde{\lambda}+2 \mu) \partial_{T T T T} Z_{3} .
$$

Using (6.6), we then compute directly the expression of $\breve{\mathcal{A}}^{0}$ which is equal to $\breve{\mathcal{K}}^{0}$.

(ii) BOUNDARY CONDITIONS. If $\boldsymbol{\zeta}\left[\varepsilon^{1 / 2}\right]=\sum_{k \geq 0} \varepsilon^{k / 2} \boldsymbol{\zeta}^{k / 2}$ is a formal series with coefficients in the space $\Sigma\left(S_{0}\right)$, we can consider the formal series (see (1.31))

$$
\mathrm{d}\left[\varepsilon^{1 / 2}\right] \boldsymbol{\zeta}\left[\varepsilon^{1 / 2}\right]=\sum_{n \geq 0} \varepsilon^{n / 2}\left(\sum_{\ell=0}^{n} \mathrm{~d}^{\ell / 2} \boldsymbol{\zeta}^{(n-\ell) / 2}\right),
$$

with coefficients in the space $\mathscr{C}^{\infty}\left(\partial S_{0}\right)^{4}$.

With the formal series $\mathrm{d}\left[\varepsilon^{1 / 2}\right]$ is associated a formal series $\boldsymbol{D}\left[\varepsilon^{1 / 2}\right]$ with operator coefficients on $S_{0}$ such that $\mathrm{d}\left[\varepsilon^{1 / 2}\right] \boldsymbol{z}=\left.\left(\boldsymbol{D}\left[\varepsilon^{1 / 2}\right] \boldsymbol{z}\right)\right|_{\partial S_{0}}$ for all $\boldsymbol{z} \in \Sigma\left(S_{0}\right)$ (see Proposition 5.4 and Equation (6.1) of [15]). As before we define the operators $\mathcal{D}^{(k)}(\varepsilon)$ on $\breve{S}:=\mathbb{R}^{+} \times \partial S_{0}$ by

$$
\forall k \geq 0 \quad \mathcal{D}^{(k)}(\varepsilon)\left(T, s ; \partial_{T}, \partial_{s}\right):=\boldsymbol{D}^{k}\left(\varepsilon^{1 / 2} T, s ; \varepsilon^{-1 / 2} \partial_{T}, \partial_{s}\right) .
$$

As in Lemma 6.1, we have the expansion

$$
\forall k \geq 0, \quad \mathcal{D}^{(k)}\left[\varepsilon^{1 / 2}\right]=\sum_{\ell \geq-(k+1)} \varepsilon^{\ell / 2} \mathcal{D}^{k, \ell / 2}
$$

Hence we can define the formal series

$$
\mathcal{D}\left[\varepsilon^{1 / 2}\right]=\sum_{k \geq 0} \varepsilon^{k} \mathcal{D}^{(k)}\left[\varepsilon^{1 / 2}\right]=\sum_{k \geq-1} \varepsilon^{n / 2} \mathcal{D}^{n / 2} \quad \text { with } \quad \mathcal{D}^{n / 2}=\sum_{k=0}^{n+1} \mathcal{D}^{k,(n-2 k) / 2} .
$$


The formal series $\boldsymbol{d}\left[\varepsilon^{1 / 2}\right]=\sum_{k \geq-1} \varepsilon^{k / 2} \boldsymbol{d}^{k / 2}$ with operators coefficients $\boldsymbol{d}^{k / 2}: \Sigma(\breve{S}) \rightarrow$ $\mathscr{C}^{\infty}\left(\partial S_{0}\right)^{4}$, is then defined by

$$
\boldsymbol{d}\left[\varepsilon^{1 / 2}\right] \boldsymbol{Z}=\left.\left(\mathcal{D}\left[\varepsilon^{1 / 2}\right] \boldsymbol{Z}\right)\right|_{T=0},
$$

for $\boldsymbol{Z} \in \Sigma(\breve{S})$. In the following we write $\boldsymbol{d}^{k / 2}=\left(d_{T}^{k / 2}, d_{s}^{k / 2}, d_{3}^{k / 2}, d_{n}^{k / 2}\right)$ the components of the operators $\boldsymbol{d}^{k / 2}$.

We have $\boldsymbol{D}^{0} \boldsymbol{z}=\left(z_{r}, z_{s}, z_{3}, \partial_{r} z_{3}\right)$. Using the orders of derivative of the operators $\boldsymbol{D}^{k}$, we see that the formal series $\boldsymbol{d}\left[\varepsilon^{1 / 2}\right]$ writes

$$
\boldsymbol{d}\left[\varepsilon^{1 / 2}\right]=\varepsilon^{-1 / 2} \boldsymbol{d}^{-1 / 2}+\boldsymbol{d}^{0}+\cdots,
$$

with

$$
\boldsymbol{d}^{-1 / 2} \boldsymbol{Z}=\left.\left(0,0,0, \partial_{T} Z_{3}\right)\right|_{T=0} \quad \text { and } \quad \boldsymbol{d}^{0} \boldsymbol{Z}=\left.\left(Z_{r}, Z_{s}, Z_{3}, d_{n}^{0}(\boldsymbol{Z})\right)\right|_{T=0} .
$$

The formal series boundary equation finally writes:

$$
\mathrm{d}\left[\varepsilon^{1 / 2}\right] \boldsymbol{\zeta}\left[\varepsilon^{1 / 2}\right]+\boldsymbol{d}\left[\varepsilon^{1 / 2}\right] \boldsymbol{Z}\left[\varepsilon^{1 / 2}\right]=\mathrm{h}\left[\varepsilon^{1 / 2}\right] \boldsymbol{f}\left[\varepsilon^{1 / 2}\right] .
$$

In order to obtain equations on $\breve{Z}\left[\varepsilon^{1 / 2}\right]$, we define the formal series $\breve{\mathcal{D}}\left[\varepsilon^{1 / 2}\right]$ and $\breve{d}\left[\varepsilon^{1 / 2}\right]$ by

$$
\breve{\mathcal{D}}\left[\varepsilon^{1 / 2}\right] \breve{\boldsymbol{Z}}\left[\varepsilon^{1 / 2}\right]:=\mathcal{D}\left[\varepsilon^{1 / 2}\right] \boldsymbol{Z}\left[\varepsilon^{1 / 2}\right], \quad \text { and } \quad \breve{\boldsymbol{d}}\left[\varepsilon^{1 / 2}\right] \breve{\boldsymbol{Z}}=\left.\left(\breve{\mathcal{D}}\left[\varepsilon^{1 / 2}\right] \breve{\boldsymbol{Z}}\right)\right|_{T=0} .
$$

Using the equations (3.9), we compute that we have

$$
\breve{\boldsymbol{d}}\left[\varepsilon^{1 / 2}\right]=\sum_{k \geq-2} \varepsilon^{k / 2} \breve{\boldsymbol{d}}^{k / 2}=\varepsilon^{-1} \breve{\boldsymbol{d}}^{-1}+\varepsilon^{-1 / 2} \breve{\boldsymbol{d}}^{-1 / 2}+\breve{\boldsymbol{d}}^{0}+\cdots,
$$

with $\breve{d}_{n}^{-1} \breve{\boldsymbol{Z}}=\left.\partial_{T} \breve{Z}_{3}\right|_{T=0}, \breve{d}_{3}^{-1} \breve{\boldsymbol{Z}}=0$ and $\breve{d}_{3}^{-1 / 2} \breve{\boldsymbol{Z}}=\left.\breve{Z}_{3}\right|_{T=0}$. Moreover we have

$$
\breve{d}_{T}^{-1} \breve{\boldsymbol{Z}}=\breve{d}_{s}^{-1} \breve{\boldsymbol{Z}}=\breve{d}_{T}^{-1 / 2} \breve{\boldsymbol{Z}}=\breve{d}_{s}^{-1 / 2} \breve{\boldsymbol{Z}}=0, \quad \breve{d}_{T}^{0} \breve{\boldsymbol{Z}}=\left.\breve{Z}_{T}\right|_{T=0} \quad \text { and } \quad \breve{d}_{s}^{0} \breve{\boldsymbol{Z}}=\left.\breve{Z}_{s}\right|_{T=0} .
$$

Grouping together the equations (6.1), (6.5) and (6.13) we define the problem: Find a formal series $\boldsymbol{\zeta}\left[\varepsilon^{1 / 2}\right]=\sum_{k \geq 0} \varepsilon^{k / 2} \boldsymbol{\zeta}^{k / 2}$ with coefficients in $\Sigma\left(S_{0}\right)$ and a formal series $\boldsymbol{Z}\left[\varepsilon^{1 / 2}\right]=\sum_{k \geq 0} \varepsilon^{k / 2} \boldsymbol{Z}^{k / 2}$ with coefficients in the space $\mathscr{C}^{\infty}\left(\partial S_{0}, \mathfrak{T}\left(\mathbb{R}^{+}\right)\right)$satisfying the relations

$$
\left\{\begin{aligned}
\mathrm{A}\left[\varepsilon^{1 / 2}\right] \boldsymbol{\zeta}\left[\varepsilon^{1 / 2}\right] & =\mathrm{G}\left[\varepsilon^{1 / 2}\right] \boldsymbol{f}\left[\varepsilon^{1 / 2}\right], \\
\boldsymbol{A}\left[\varepsilon^{1 / 2}\right] \boldsymbol{Z}\left[\varepsilon^{1 / 2}\right] & =0, \\
\mathrm{~d}\left[\varepsilon^{1 / 2}\right] \boldsymbol{\zeta}\left[\varepsilon^{1 / 2}\right]+\boldsymbol{d}\left[\varepsilon^{1 / 2}\right] \boldsymbol{Z}\left[\varepsilon^{1 / 2}\right] & =\mathrm{h}\left[\varepsilon^{1 / 2}\right] \boldsymbol{f}\left[\varepsilon^{1 / 2}\right] .
\end{aligned}\right.
$$




\section{B SOLUTION OF THE FORMAL SERIES PROBLEM}

We now prove that the system (6.15) admits a solution. The method is close to the proof of Theorem 4.1.

Theorem 6.3 Suppose that $S_{0}$ is elliptic, and let $\boldsymbol{f}\left[\varepsilon^{1 / 2}\right]$ be a formal series with coefficients in $\mathscr{C}^{\infty}\left(I, \Sigma\left(S_{0}\right)\right)$. Then there exists a unique formal series $\zeta\left[\varepsilon^{1 / 2}\right]$ with coefficients in the space $\Sigma\left(S_{0}\right)$ and a unique formal series $\boldsymbol{Z}\left[\varepsilon^{1 / 2}\right]$ with coefficients in the space $\mathscr{C}^{\infty}\left(\partial S_{0}, \mathfrak{T}\left(\mathbb{R}^{+}\right)\right)$satisfying the equation (6.15).

Proof. We set $\boldsymbol{g}\left[\varepsilon^{1 / 2}\right]:=\mathrm{G}\left[\varepsilon^{1 / 2}\right] \boldsymbol{f}\left[\varepsilon^{1 / 2}\right]$ and $\boldsymbol{c}\left[\varepsilon^{1 / 2}\right]:=\mathrm{h}\left[\varepsilon^{1 / 2}\right] \boldsymbol{f}\left[\varepsilon^{1 / 2}\right]$. The coefficients $\boldsymbol{g}^{k / 2}$ and $\boldsymbol{c}^{k / 2}$ are thus in $\Sigma\left(S_{0}\right)$ and $\mathscr{C}^{\infty}\left(\partial S_{0}\right)^{4}$ respectively. Note that $\boldsymbol{c}^{0}=0$ because $\mathrm{h}^{0}$ is the zero operator (see Theorem 5.3 and Equation (6.1) of [15]).

The last equation of (6.15) is equivalent to

$$
\mathrm{d}\left[\varepsilon^{1 / 2}\right] \boldsymbol{\zeta}\left[\varepsilon^{1 / 2}\right]+\breve{\boldsymbol{d}}\left[\varepsilon^{1 / 2}\right] \breve{\boldsymbol{Z}}\left[\varepsilon^{1 / 2}\right]=\mathrm{h}\left[\varepsilon^{1 / 2}\right] \boldsymbol{f}\left[\varepsilon^{1 / 2}\right] .
$$

This writes, for $k \geq-2$,

$$
\sum_{\ell=0}^{k} \mathrm{~d}^{\ell / 2}\left(\boldsymbol{\zeta}^{(k-\ell) / 2}\right)+\breve{\boldsymbol{d}}^{-1}\left(\breve{\boldsymbol{Z}}^{(k+2) / 2}\right)+\breve{\boldsymbol{d}}^{-1 / 2}\left(\breve{\boldsymbol{Z}}^{(k+1) / 2}\right)+\sum_{\ell=0}^{k} \breve{\boldsymbol{d}}^{\ell / 2}\left(\breve{\boldsymbol{Z}}^{(k-\ell) / 2}\right)=\boldsymbol{c}^{k / 2} .
$$

Using the expressions of the first operators, we get:

$$
\left.\zeta_{r}^{k / 2}\right|_{\partial S_{0}}+\sum_{\ell=1}^{k} \mathrm{~d}_{r}^{\ell / 2}\left(\boldsymbol{\zeta}^{(k-\ell) / 2}\right)+\left.\breve{Z}_{T}^{k / 2}\right|_{T=0}+\sum_{\ell=1}^{k} \breve{d}_{T}^{\ell / 2}\left(\breve{\boldsymbol{Z}}^{(k-\ell) / 2}\right)=c_{T}^{k / 2},
$$

and

$$
\left.\zeta_{s}^{k / 2}\right|_{\partial S_{0}}+\sum_{\ell=1}^{k} \mathrm{~d}_{s}^{\ell / 2}\left(\boldsymbol{\zeta}^{(k-\ell) / 2}\right)+\left.\breve{Z}_{s}^{k / 2}\right|_{T=0}+\sum_{\ell=1}^{k} \breve{d}_{s}^{\ell / 2}\left(\breve{\boldsymbol{Z}}^{(k-\ell) / 2}\right)=c_{s}^{k / 2},
$$

for the surfacic components. For the transverse components we get

$$
\left.\zeta_{3}^{k / 2}\right|_{\partial S_{0}}+\sum_{\ell=1}^{k} \mathrm{~d}_{3}^{\ell / 2}\left(\boldsymbol{\zeta}^{(k-\ell) / 2}\right)+\left.\breve{Z}_{3}^{(k+1) / 2}\right|_{T=0}+\sum_{\ell=0}^{k} \breve{d}_{3}^{\ell / 2}\left(\breve{\boldsymbol{Z}}^{(k-\ell) / 2}\right)=c_{3}^{k / 2},
$$

and similarly,

$$
\left.\partial_{r} \zeta_{3}^{k / 2}\right|_{\partial S_{0}}+\sum_{\ell=1}^{k} \mathrm{~d}_{n}^{\ell / 2}\left(\boldsymbol{\zeta}^{(k-\ell) / 2}\right)+\left.\partial_{T} \breve{Z}_{3}^{(k+2) / 2}\right|_{T=0}+\sum_{\ell=-1}^{k} \breve{d}_{n}^{\ell / 2}\left(\breve{\boldsymbol{Z}}^{(k-\ell) / 2}\right)=c_{n}^{k / 2} .
$$

Note that these last two equations express $\left.\breve{Z}_{3}^{(k+1) / 2}\right|_{T=0}$ and $\left.\partial_{T} \breve{Z}_{3}^{(k+2) / 2}\right|_{T=0}$ with respect to terms of lower order in $k$. Moreover, these two terms correspond to the boundary conditions we can impose when solving for the operator $\breve{\mathcal{A}}^{0}$. Finally, the formal series 
$\boldsymbol{\zeta}\left[\varepsilon^{1 / 2}\right]$ and $\boldsymbol{Z}\left[\varepsilon^{1 / 2}\right]$ solve the reduced formal series problem (6.15) if for $k \geq 0$ we have:

$$
\begin{aligned}
\breve{\mathcal{A}}^{0}\left(\breve{\boldsymbol{Z}}^{k / 2}\right) & =-\sum_{\ell=1}^{k} \breve{\mathcal{A}}^{\ell / 2}\left(\breve{\boldsymbol{Z}}^{(k-\ell) / 2}\right) \text { in } \breve{S}, \\
\left.\breve{Z}_{3}^{k / 2}\right|_{T=0} & =c_{3}^{(k-1) / 2}-\sum_{\ell=1}^{k} \breve{d}_{3}^{(\ell-1) / 2}\left(\breve{\boldsymbol{Z}}^{(k-\ell) / 2}\right)-\sum_{\ell=0}^{k-1} \mathrm{~d}_{3}^{\ell / 2}\left(\boldsymbol{\zeta}^{(k-1-\ell) / 2}\right), \\
\left.\partial_{T} \breve{Z}_{3}^{k / 2}\right|_{T=0} & =c_{n}^{(k-2) / 2}-\sum_{\ell=1}^{k} \breve{d}_{n}^{(\ell-2) / 2}\left(\breve{\boldsymbol{Z}}^{(k-\ell) / 2}\right)-\sum_{\ell=0}^{k-2} \mathrm{~d}_{n}^{\ell / 2}\left(\boldsymbol{\zeta}^{(k-2-\ell) / 2}\right),
\end{aligned}
$$

and

$$
\begin{aligned}
& \mathrm{M}\left(\boldsymbol{\zeta}^{k / 2}\right)=-\sum_{\ell=1}^{k} \mathrm{~A}^{\ell / 2}\left(\boldsymbol{\zeta}^{(k-\ell) / 2}\right)+\boldsymbol{g}^{k / 2} \text { in } S_{0}, \\
& \left.\zeta_{r}^{k / 2}\right|_{\partial S_{0}}=c_{r}^{k / 2}-\sum_{\ell=1}^{k} \mathrm{~d}_{r}^{\ell / 2}\left(\boldsymbol{\zeta}^{(k-\ell) / 2}\right)-\left.\breve{Z}_{T}^{k / 2}\right|_{T=0}-\sum_{\ell=1}^{k} \breve{d}_{T}^{\ell / 2}\left(\breve{\boldsymbol{Z}}^{(k-\ell) / 2}\right), \\
& \left.\zeta_{s}^{k / 2}\right|_{\partial S_{0}}=c_{s}^{k / 2}-\sum_{\ell=1}^{k} \mathrm{~d}_{s}^{\ell / 2}\left(\boldsymbol{\zeta}^{(k-\ell) / 2}\right)-\left.\breve{Z}_{s}^{k / 2}\right|_{T=0}-\sum_{\ell=1}^{k} \breve{d}_{s}^{\ell / 2}\left(\breve{\boldsymbol{Z}}^{(k-\ell) / 2}\right),
\end{aligned}
$$

where we agree that $\boldsymbol{c}^{\ell / 2}, \boldsymbol{\zeta}^{\ell / 2}$ and $Z^{\ell / 2}$ are zero for $\ell \leq 0$.

The end of the proof is similar to the proof of Theorem 4.1, using the theorems 2.2 and 3.2 and the fact that $\mathcal{A}^{0}=\mathcal{K}^{0}$.

\section{C COMParison With THE KoITER MODEL}

Proposition 6.4 Let $\left(\zeta\left[\varepsilon^{1 / 2}\right], Z\left[\varepsilon^{1 / 2}\right]\right)$ be the solution of the problem (6.15) given by Theorem 6.3 and $\left(\zeta^{\prime}\left[\varepsilon^{1 / 2}\right], Z^{\prime}\left[\varepsilon^{1 / 2}\right]\right)$ the solution of the system

$$
\left\{\begin{aligned}
\mathrm{K}\left[\varepsilon^{1 / 2}\right] \boldsymbol{\zeta}^{\prime}\left[\varepsilon^{1 / 2}\right] & =\mathrm{G}^{0} \boldsymbol{f}\left[\varepsilon^{1 / 2}\right], \\
\mathcal{K}\left[\varepsilon^{1 / 2}\right] \boldsymbol{Z}^{\prime}\left[\varepsilon^{1 / 2}\right] & =0, \\
\mathrm{~d}^{0} \boldsymbol{\zeta}^{\prime}\left[\varepsilon^{1 / 2}\right]+\left(d_{T}^{0}, d_{s}^{0}, d_{3}^{0}, \varepsilon^{-1 / 2} d_{n}^{-1 / 2}\right) \boldsymbol{Z}^{\prime}\left[\varepsilon^{1 / 2}\right] & =0,
\end{aligned}\right.
$$

given by Theorem 4.1. Then we have

$$
\boldsymbol{\zeta}\left[\varepsilon^{1 / 2}\right]-\boldsymbol{\zeta}^{\prime}\left[\varepsilon^{1 / 2}\right]=\varepsilon \boldsymbol{e}^{1}+\sum_{k \geq 3} \varepsilon^{k / 2} \boldsymbol{e}^{k / 2}
$$

and

$$
\boldsymbol{Z}\left[\varepsilon^{1 / 2}\right]-\boldsymbol{Z}^{\prime}\left[\varepsilon^{1 / 2}\right]=\left(0, \varepsilon^{1 / 2} E_{3}^{1 / 2}\right)+\sum_{k \geq 2} \varepsilon^{k / 2} \boldsymbol{E}^{k / 2}
$$

where $\boldsymbol{e}^{k / 2} \in \Sigma\left(S_{0}\right)$ and $\boldsymbol{E}^{k / 2} \in \mathscr{C}^{\infty}\left(\partial S_{0}, \boldsymbol{T}\left(\mathbb{R}^{+}\right)\right)$.

Proof. We set

$$
\boldsymbol{g}\left[\varepsilon^{1 / 2}\right]=\mathrm{G}\left[\varepsilon^{1 / 2}\right] \boldsymbol{f}\left[\varepsilon^{1 / 2}\right] \text { and } \boldsymbol{c}\left[\varepsilon^{1 / 2}\right]=\mathrm{h}\left[\varepsilon^{1 / 2}\right] \boldsymbol{f}\left[\varepsilon^{1 / 2}\right] .
$$

We easily see that we have $\boldsymbol{c}^{0}=0, \boldsymbol{c}^{1 / 2}=0$ and $\boldsymbol{g}^{1 / 2}=0$. 
Comparing the proofs of Theorems 4.1 and 6.3, we see that the two solutions have the same first terms $\breve{Z}^{0}=0$ and $\boldsymbol{\zeta}^{0}$ solution of the system

$$
\mathrm{M}\left(\boldsymbol{\zeta}^{0}\right)=\mathrm{G}^{0} \boldsymbol{f}^{0}=\frac{1}{2} \int_{-1}^{1} \boldsymbol{f}^{0} \mathrm{~d} x_{3} \quad \text { in } \quad S_{0}, \quad \text { and }\left.\quad \zeta_{r}\right|_{\partial S_{0}}=\left.\zeta_{s}\right|_{\partial S_{0}}=0 .
$$

For $k=1$, the equations (6.16) write:

$$
\breve{\mathcal{A}}^{0}\left(\breve{Z}^{1 / 2}\right)=0 \quad \text { in } \quad \breve{S},\left.\quad \breve{Z}_{3}^{1 / 2}\right|_{T=0}=-\left.\zeta_{3}^{0}\right|_{\partial S_{0}}, \quad \text { and }\left.\quad \partial_{T} \breve{Z}_{3}^{1 / 2}\right|_{T=0}=0,
$$

and using $\breve{\mathcal{K}}^{0}=\breve{\mathcal{A}}^{0}$, we see that this system is identical to the first system of (4.9) with the right-hand sides $\breve{G}^{0}=0$ and $c_{3}^{0}=0$. We deduce that the term $\breve{Z}^{1 / 2}$ solution of the previous system is the same for both solutions of (6.15) and (6.18).

The equations (6.17) then write

$$
\mathrm{M}\left(\boldsymbol{\zeta}^{1 / 2}\right)=0 \quad \text { in } \quad S_{0},\left.\quad \zeta_{r}^{1 / 2}\right|_{\partial S_{0}}=-\left.\breve{Z}_{T}^{1 / 2}\right|_{T=0}, \quad \text { and }\left.\quad \zeta_{s}^{1 / 2}\right|_{\partial S_{0}}=-\left.\breve{Z}_{s}^{1 / 2}\right|_{T=0},
$$

and we verify that the equations (4.7) for $k=1$ are the same. The term $\zeta^{1 / 2}$ solution of this system is then common in both formal series $\zeta\left[\varepsilon^{1 / 2}\right]$ and $\zeta^{\prime}\left[\varepsilon^{1 / 2}\right]$.

For $k=2$, the equations (6.16) write $\breve{\mathcal{A}}^{0}\left(\breve{Z}^{1}\right)=-\breve{\mathcal{A}}^{1 / 2}\left(\breve{\boldsymbol{Z}}^{1 / 2}\right)$ with

$$
\left.\breve{Z}_{3}^{1}\right|_{T=0}=-\breve{d}_{3}^{0}\left(\breve{\boldsymbol{Z}}^{1 / 2}\right)-\left.\zeta_{3}^{1 / 2}\right|_{\partial S_{0}} \quad \text { and }\left.\quad \partial_{T} \breve{Z}_{3}^{1}\right|_{T=0}=-\breve{d}_{n}^{-1 / 2}\left(\breve{\boldsymbol{Z}}^{1 / 2}\right)-\left.\partial_{r} \zeta_{3}^{0}\right|_{\partial S_{0}},
$$

and the equation (4.6) associated with the problem (6.18) writes

$$
\breve{\mathcal{K}}^{0}\left(\breve{\boldsymbol{Z}}^{\prime 1}\right)=-\breve{\mathcal{K}}^{1 / 2}\left(\breve{\boldsymbol{Z}}^{\prime 1 / 2}\right),\left.\quad \breve{Z}_{3}^{\prime 1}\right|_{T=0}=-\left.\zeta_{3}^{\prime 1 / 2}\right|_{\partial S_{0}} \quad \text { and }\left.\quad \partial_{T} \breve{Z}_{3}^{\prime 1}\right|_{T=0}=-\left.\partial_{r} \zeta_{3}^{\prime 0}\right|_{\partial S_{0}} .
$$

The proposition is a consequence of the following result, proved in Appendix A:

Lemma 6.5 The operators $\breve{\mathcal{A}}^{1 / 2}$ and $\breve{\mathcal{K}}^{1 / 2}$ are the same, the operator $\breve{d}_{3}^{0}$ is the zero operator, and the operator $\breve{d}_{n}^{-1 / 2}$ writes

$$
\breve{d}_{n}^{-1 / 2}(\breve{\boldsymbol{Z}})=\left.c_{2} \partial_{T T} \breve{Z}_{3}\right|_{T=0} .
$$

where $c_{2}>0$ is a constant depending only on the Lamé coefficients $\lambda$ and $\mu$.

The operator (6.19) being not equal to the zero operator, the terms $\breve{Z}^{1}$ and $\breve{Z}^{\prime 1}$ do differ in general. This implies that the terms $\zeta^{1}$ and $\zeta^{\prime 1}$ also differ. This proves the proposition.

\section{THREE-DIMENSIONAL FORMAL SERIES SOLUTION}

We recall the definition of the three-dimensional boundary layer spaces ([11, 8, 15]): After the change of coordinate $R=\varepsilon^{-1} r$ in a neighborhood of $\Gamma_{0}$, the coordinate system $\left(R, X_{3}, s\right)$ is defined on the manifold $\Sigma^{+} \times \partial S_{0}$ where $\Sigma^{+}:=\mathbb{R}^{+} \times I \ni\left(R, X_{3}\right)$ is a semi-strip. Let $\mathfrak{H}\left(\Sigma^{+}\right)$be the space of $\mathscr{C}^{\infty}$ functions $\varphi$ on the semi-strip $\Sigma^{+}$except in 
the non regular points $\left(R=0, X_{3}= \pm 1\right)$, and such that $\varphi$ is exponentially decreasing with $R$ in the following sense:

$$
\forall i, j, k \in \mathbb{N}, \quad e^{\delta R} R^{k} \partial_{R}^{i} \partial_{X_{3}}^{j} \varphi \in \mathrm{L}^{2}\left(\Sigma^{+}\right),
$$

where $\delta>0$ is a real strictly less than the smallest Papkovich-Fadle exponent (see [17]). In the neighborhood of the two corners of the semi-strip, we impose the following: if $\rho$ denote the distance in $\Sigma^{+}$to a point $\left(R=0, X_{3}= \pm 1\right)$, we suppose that each $\varphi$ in $\mathfrak{H}\left(\Sigma^{+}\right)$satisfies

$$
\forall i, j \in \mathbb{N}, i+j \neq 0, \quad \rho^{i+j-1} \partial_{R}^{i} \partial_{X_{3}}^{j} \varphi \in \mathrm{L}^{2}\left(\Sigma^{+}\right) .
$$

We then define the corresponding displacement space

$$
\mathfrak{H}\left(\Sigma^{+}\right):=\left\{\boldsymbol{\varphi}=\left(\varphi_{R}, \varphi_{s}, \varphi_{3}\right) \in \mathfrak{H}\left(\Sigma^{+}\right)^{3}\right\} .
$$

In this section, we construct three formal series:

A formal series $\boldsymbol{v}\left[\varepsilon^{1 / 2}\right]=\sum_{k \geq 0} \varepsilon^{k / 2} \boldsymbol{v}^{k / 2}$ with coefficients

$$
\boldsymbol{v}^{k / 2}\left(x_{1}, x_{2}, X_{3}\right) \in \mathscr{C}^{\infty}\left(I, \Sigma\left(S_{0}\right)\right),
$$

a formal series $\boldsymbol{W}\left[\varepsilon^{1 / 2}\right]:=\sum_{k \geq 0} \varepsilon^{k / 2} \boldsymbol{W}^{k / 2}$ with coefficients

$$
\boldsymbol{W}^{k / 2}\left(T, s, X_{3}\right) \in \mathscr{C}^{\infty}\left(I \times \partial S_{0}, \mathfrak{T}\left(\mathbb{R}^{+}\right)\right),
$$

and a formal series $\boldsymbol{\Phi}\left[\varepsilon^{1 / 2}\right]=\sum_{k \geq 0} \varepsilon^{k / 2} \boldsymbol{\Phi}^{k / 2}$ with coefficients

$$
\boldsymbol{\Phi}^{k / 2}\left(R, s, X_{3}\right) \in \mathscr{C}^{\infty}\left(\partial S_{0}, \mathfrak{H}\left(\Sigma^{+}\right)\right),
$$

solutions a the $3 \mathrm{D}$ formal series equations in the corresponding coordinates, and satisfying the boundary condition:

$$
\left.\boldsymbol{v}\left[\varepsilon^{1 / 2}\right]\right|_{\Gamma_{0}}+\left.\boldsymbol{W}\left[\varepsilon^{1 / 2}\right]\right|_{T=0}+\left.\boldsymbol{\Phi}\left[\varepsilon^{1 / 2}\right]\right|_{R=0}=0 .
$$

These three formal series are constructed using the formal series reduction of [15], and the formal series obtained by Theorem 6.3. To this aim, we define the action of the operator formal series $\mathrm{V}\left[\varepsilon^{1 / 2}\right]$ and $\Psi\left[\varepsilon^{1 / 2}\right]$ (see Theorem 5.3 of [15]) on the 2D boundary layer formal series, taking values in $\mathscr{C}^{\infty}\left(I \times \partial S_{0}, \mathfrak{T}\left(\mathbb{R}^{+}\right)\right)$. From now on, $\boldsymbol{\zeta}\left[\varepsilon^{1 / 2}\right]$ and $\boldsymbol{Z}\left[\varepsilon^{1 / 2}\right]$ are the formal series given by Theorem 6.3 .

\section{A CONSTRUCTION OF THE FORMAL SERIES}

(i) Construction of $\boldsymbol{v}\left[\varepsilon^{1 / 2}\right]$. We define the formal series

$$
\boldsymbol{v}\left[\varepsilon^{1 / 2}\right]=\mathrm{V}\left[\varepsilon^{1 / 2}\right] \boldsymbol{\zeta}\left[\varepsilon^{1 / 2}\right]+\mathrm{Q}\left[\varepsilon^{1 / 2}\right] \boldsymbol{f}\left[\varepsilon^{1 / 2}\right]
$$

with coefficients in the space $\mathscr{C}^{\infty}\left(I, \Sigma\left(S_{0}\right)\right)$. As $\boldsymbol{\zeta}\left[\varepsilon^{1 / 2}\right]$ is solution of the equation

$$
\mathrm{A}\left[\varepsilon^{1 / 2}\right] \boldsymbol{\zeta}\left[\varepsilon^{1 / 2}\right]=\mathrm{G}\left[\varepsilon^{1 / 2}\right] \boldsymbol{f}\left[\varepsilon^{1 / 2}\right],
$$

we easily see using (1.27) that we have

$$
\mathrm{L}\left[\varepsilon^{1 / 2}\right] \boldsymbol{v}\left[\varepsilon^{1 / 2}\right]=-\boldsymbol{f}\left[\varepsilon^{1 / 2}\right] \text { and } \mathrm{T}\left[\varepsilon^{1 / 2}\right] \boldsymbol{v}\left[\varepsilon^{1 / 2}\right]=0 \text {. }
$$


(ii) Construction of $\boldsymbol{W}\left[\varepsilon^{1 / 2}\right]$. In order to define the formal series $\boldsymbol{W}\left[\varepsilon^{1 / 2}\right]$, we make the change of variable in the operator formal series $\mathrm{V}\left[\varepsilon^{1 / 2}\right]$. Recall that the operators $\mathrm{V}^{k / 2}$ vanish if $k$ is odd. If we denote by $\mathrm{V}^{k}\left(r, s, X_{3} ; \partial_{r}, \partial_{s}\right)$ the operator $\mathrm{V}^{k}$, polynomial in $X_{3}$, in the coordinate system $\left(r, s, X_{3}\right)$, then we set for all $k \geq 0$,

$$
\mathcal{V}^{(k)}(\varepsilon) \boldsymbol{Z}=\mathrm{V}^{k}\left(\varepsilon^{1 / 2} T, s, X_{3} ; \varepsilon^{-1 / 2} \partial_{T}, \partial_{s}\right)(\boldsymbol{Z}),
$$

where $Z$ is a 1 -form field on the manifold $\breve{S}$. As in Lemma 6.1 the corresponding formal series $\mathcal{V}^{(k)}\left[\varepsilon^{1 / 2}\right]$ obtained by Taylor expansion in $T=0$ of the coefficients write

$$
\mathcal{V}^{(k)}\left[\varepsilon^{1 / 2}\right]=\sum_{\ell \geq-k} \varepsilon^{\ell / 2} \mathcal{V}^{k, \ell / 2}
$$

where the operators $\mathcal{V}^{k, \ell / 2}$ are polynomial in $T$ and $X_{3}$ and act on $\Sigma(\breve{S})$ (see [15]). We define the formal series $\mathcal{V}\left[\varepsilon^{1 / 2}\right]$ by the equation

$$
\mathcal{V}\left[\varepsilon^{1 / 2}\right]:=\sum_{k \geq 0} \varepsilon^{k} \mathcal{V}^{(k)}\left[\varepsilon^{1 / 2}\right]=\sum_{n \geq 0} \varepsilon^{n / 2} \mathcal{V}^{n / 2}, \quad \text { with } \quad \mathcal{V}^{n / 2}=\sum_{k=0}^{n} \mathcal{V}^{k,(n-2 k) / 2}
$$

Using the definition of the space $\mathfrak{T}\left(\mathbb{R}^{+}\right)$, , we see that the formal series $\mathcal{V}\left[\varepsilon^{1 / 2}\right]$ has operator coefficients

$$
\mathcal{V}^{n / 2}: \mathscr{C}^{\infty}\left(\partial S_{0}, \mathfrak{T}\left(\mathbb{R}^{+}\right)\right) \rightarrow \mathscr{C}^{\infty}\left(I \times \partial S_{0}, \mathfrak{T}\left(\mathbb{R}^{+}\right)\right)
$$

polynomial in $T$ and $X_{3}$. We have $\mathcal{V}^{0} \boldsymbol{Z}=\boldsymbol{Z}$. We now define the formal series

$$
\boldsymbol{W}\left[\varepsilon^{1 / 2}\right]:=\mathcal{V}\left[\varepsilon^{1 / 2}\right] \boldsymbol{Z}\left[\varepsilon^{1 / 2}\right]
$$

with coefficients in the space $\mathscr{C}^{\infty}\left(I \times \partial S_{0}, \mathfrak{T}\left(\mathbb{R}^{+}\right)\right)$.

(iii) Construction of $\boldsymbol{\Phi}\left[\varepsilon^{1 / 2}\right]$. Recall that Theorem 5.3 in [15] yields the existence of a formal series $\Psi[\varepsilon]$ mapping $\Sigma\left(S_{0}\right)$ to the space of three-dimensional boundary layers $\mathscr{C}^{\infty}\left(\partial S_{0}, \mathfrak{H}\left(\Sigma^{+}\right)\right)$.

We first note that we can consider the formal series $\Psi\left[\varepsilon^{1 / 2}\right] \boldsymbol{\zeta}\left[\varepsilon^{1 / 2}\right]$ with boundary layer coefficients. We now define the action of the formal series $\Psi\left[\varepsilon^{1 / 2}\right]$ on the formal series $\boldsymbol{Z}\left[\varepsilon^{1 / 2}\right]$.

The formal series $\Psi\left[\varepsilon^{1 / 2}\right]$ contains only terms in even powers of $\varepsilon^{1 / 2}$ and we have (see Proposition 5.4 in [15])

$$
\forall k \geq 0, \quad \Psi^{k} \boldsymbol{z}=\left.\sum_{j \in F_{k}}\left(P_{j}^{k} \boldsymbol{z}\right)\right|_{\partial S_{0}} \varphi^{k, j}
$$

where $F_{k}$ is a finite subset of $\mathbb{N}$. The coefficients $\varphi^{k, j}\left(R, s, x_{3}\right)$ are in the space $\mathscr{C}^{\infty}\left(\partial S_{0}, \mathfrak{H}\left(\Sigma^{+}\right)\right)$and the operators $P_{j}^{k}$ take values in $\mathscr{C}^{\infty}\left(S_{0}\right)$ and are of order at most $k$ in $\partial_{r}$. As in the lemma 6.1, we define for all $Z \in \Sigma(\breve{S})$,

$$
\mathcal{P}_{j}^{(k)}(\varepsilon) \boldsymbol{Z}=P_{j}^{k}\left(\varepsilon^{1 / 2} T, s ; \varepsilon^{-1 / 2} \partial_{T}, \partial_{s}\right)(\boldsymbol{Z}),
$$


and the associated formal series $\mathcal{P}_{j}^{(k)}\left[\varepsilon^{1 / 2}\right]$ after expansion of the coefficients around $T=0$. As $P_{j}^{k}$ is of order $k$ in $\partial_{r}$ we can define the formal series $\psi\left[\varepsilon^{1 / 2}\right]$ with operator coefficients

$$
\psi^{k / 2}: \mathscr{C}^{\infty}\left(\partial S_{0}, \mathfrak{T}\left(\mathbb{R}^{+}\right)\right) \rightarrow \mathscr{C}^{\infty}\left(\partial S_{0}, \mathfrak{H}\left(\Sigma^{+}\right)\right),
$$

by the equation (recall that the $F_{k}$ are finite sets)

$$
\begin{aligned}
\psi\left[\varepsilon^{1 / 2}\right] \boldsymbol{Z} & =\left.\sum_{k \geq 0} \varepsilon^{k} \sum_{j \in F_{k}}\left(\mathcal{P}_{j}^{(k)}\left[\varepsilon^{1 / 2}\right] \boldsymbol{Z}\right)\right|_{T=0} \boldsymbol{\varphi}^{k, j} \\
& =\left.\sum_{n \geq 0} \varepsilon^{n / 2} \sum_{k=0}^{n} \sum_{j \in F_{k}}\left(\mathcal{P}_{j}^{k,(\ell-2 k) / 2} \boldsymbol{Z}\right)\right|_{T=0} \boldsymbol{\varphi}^{k,(\ell-2 k) / 2} .
\end{aligned}
$$

We then define the formal series $\boldsymbol{\Phi}\left[\varepsilon^{1 / 2}\right]$ with coefficients in $\mathscr{C}^{\infty}\left(\partial S_{0}, \mathfrak{H}\left(\Sigma^{+}\right)\right)$by

$$
\boldsymbol{\Phi}\left[\varepsilon^{1 / 2}\right]:=\Psi\left[\varepsilon^{1 / 2}\right] \boldsymbol{\zeta}\left[\varepsilon^{1 / 2}\right]+\Theta\left[\varepsilon^{1 / 2}\right] \boldsymbol{f}\left[\varepsilon^{1 / 2}\right]+\psi\left[\varepsilon^{1 / 2}\right] \boldsymbol{Z}\left[\varepsilon^{1 / 2}\right] .
$$

where $\Theta[\varepsilon]$ is the formal series given by Theorem 5.3 of [15].

\section{B THREE DIMENSIONAL FORMAL SERIES EQUATION}

In the coordinate system $\left(T, s, X_{3}\right)$, we define the operator $(\mathcal{L}(\varepsilon), \mathcal{T}(\varepsilon))$ by:

$$
\left\{\begin{array}{l}
\mathcal{L}(\varepsilon)\left(T, s, X_{3} ; \partial_{T}, \partial_{s}, \partial_{X_{3}}\right):=\mathrm{L}\left(\varepsilon^{1 / 2} T, s, \varepsilon X_{3} ; \varepsilon^{-1 / 2} \partial_{T}, \partial_{s}, \varepsilon^{-1} \partial_{X_{3}}\right) \quad \text { and } \\
\mathcal{T}(\varepsilon)\left(T, s, X_{3} ; \partial_{T}, \partial_{s}, \partial_{X_{3}}\right):=\mathrm{T}\left(\varepsilon^{1 / 2} T, s, \varepsilon X_{3} ; \varepsilon^{-1 / 2} \partial_{T}, \partial_{s}, \varepsilon^{-1} \partial_{X_{3}}\right)
\end{array}\right.
$$

where $(L, T)$ is the 3D elasticity operator on $\Omega^{\varepsilon}$. We then define the two corresponding formal series $\left(\mathcal{L}\left[\varepsilon^{1 / 2}\right], \mathcal{T}\left[\varepsilon^{1 / 2}\right]\right)$ in powers of $\varepsilon^{1 / 2}$ by expanding the coefficients of this operator in $T=0$ and $X_{3}=0$. It is clear that we have operators of order 2 , polynomial in $T$ and $X_{3}$

$$
\mathcal{L}^{k / 2}: \mathscr{C}^{\infty}\left(I \times \partial S_{0}, \mathfrak{T}\left(\mathbb{R}^{+}\right)\right) \rightarrow \mathscr{C}^{\infty}\left(I \times \partial S_{0}, \mathfrak{T}\left(\mathbb{R}^{+}\right)\right),
$$

and

$$
\mathcal{T}^{k / 2}: \mathscr{C}^{\infty}\left(I \times \partial S_{0}, \mathfrak{T}\left(\mathbb{R}^{+}\right)\right) \rightarrow \mathscr{C}^{\infty}\left(\partial S_{0}, \mathfrak{T}\left(\mathbb{R}^{+}\right)\right)
$$

such that

$$
\mathcal{L}\left[\varepsilon^{1 / 2}\right]=\varepsilon^{-2} \sum_{k \geq 0} \varepsilon^{k / 2} \mathcal{L}^{k / 2} \quad \text { and } \quad \mathcal{T}\left[\varepsilon^{1 / 2}\right]=\varepsilon^{-2} \sum_{k \geq 0} \varepsilon^{k / 2} \mathcal{T}^{k / 2}
$$

In the same way, the change of variable $\left(r, s, x_{3}\right) \mapsto\left(R, s, X_{3}\right)$ where $R=\varepsilon^{-1} r$ in the operator $(\mathrm{L}, \mathrm{T})$ yields formal series $(\mathscr{L}[\varepsilon], \mathscr{T}[\varepsilon]$ ) (see the equation (5.5) in [15]).

We thus have the 3 couples of formal series: $\left(\mathrm{L}\left[\varepsilon^{1 / 2}\right], \mathrm{T}\left[\varepsilon^{1 / 2}\right]\right),\left(\mathcal{L}\left[\varepsilon^{1 / 2}\right], \mathcal{T}\left[\varepsilon^{1 / 2}\right]\right)$ and $\left(\mathscr{L}\left[\varepsilon^{1 / 2}\right], \mathscr{T}\left[\varepsilon^{1 / 2}\right]\right)$ corresponding to the expansion in powers of $\varepsilon^{1 / 2}$ of the operator $(\mathrm{L}, \mathrm{T})$ in the three coordinate systems associated with the three kind of terms. 
Theorem 7.1 The formal series $\boldsymbol{v}\left[\varepsilon^{1 / 2}\right], \boldsymbol{W}\left[\varepsilon^{1 / 2}\right]$ and $\boldsymbol{\Phi}\left[\varepsilon^{1 / 2}\right]$ constructed in the previous subsection from the solution $\left(\zeta\left[\varepsilon^{1 / 2}\right], Z\left[\varepsilon^{1 / 2}\right]\right)$ of the problem (6.15), are solutions of the following equations: the formal series $\boldsymbol{v}\left[\varepsilon^{1 / 2}\right]$ satisfies

$$
\left(\mathrm{L}\left[\varepsilon^{1 / 2}\right], \mathrm{T}\left[\varepsilon^{1 / 2}\right]\right) \boldsymbol{v}\left[\varepsilon^{1 / 2}\right]=-\left(\boldsymbol{f}\left[\varepsilon^{1 / 2}\right], 0\right)
$$

the formal series $\boldsymbol{W}\left[\varepsilon^{1 / 2}\right]$ satisfies

$$
\left(\mathcal{L}\left[\varepsilon^{1 / 2}\right], \mathcal{T}\left[\varepsilon^{1 / 2}\right]\right) \boldsymbol{W}\left[\varepsilon^{1 / 2}\right]=0,
$$

and the formal series $\boldsymbol{\Phi}\left[\varepsilon^{1 / 2}\right]$ satisfies

$$
\left(\mathscr{L}\left[\varepsilon^{1 / 2}\right], \mathscr{T}\left[\varepsilon^{1 / 2}\right]\right) \Phi\left[\varepsilon^{1 / 2}\right]=0 .
$$

Moreover, we have the relation

$$
\left.\boldsymbol{v}\left[\varepsilon^{1 / 2}\right]\right|_{\Gamma_{0}}+\left.\boldsymbol{W}\left[\varepsilon^{1 / 2}\right]\right|_{T=0}+\left.\boldsymbol{\Phi}\left[\varepsilon^{1 / 2}\right]\right|_{R=0}=0 .
$$

Proof. The equation (7.4) is clearly satisfied.

The formal series $\boldsymbol{Z}\left[\varepsilon^{1 / 2}\right]$ satisfies the equation (6.5). But by definition, the formal series $\mathrm{A}[\varepsilon]$ and $\mathrm{V}[\varepsilon]$ satisfy the first group of equations in (1.27). By doing the change of variable $\left(r, s, x_{3}\right) \mapsto\left(T, s, X_{3}\right)$ and by expanding the coefficients in $T=0$ and $X_{3}=$ 0 , we see that for all 1 -form field $Z$ on the manifold $\breve{S}$, we have

$$
\mathcal{L}\left[\varepsilon^{1 / 2}\right] \mathcal{V}\left[\varepsilon^{1 / 2}\right] \boldsymbol{Z}=-\mathcal{I} \circ \mathcal{A}\left[\varepsilon^{1 / 2}\right] \boldsymbol{Z} \quad \text { and } \quad \boldsymbol{T}\left[\varepsilon^{1 / 2}\right] \mathcal{V}\left[\varepsilon^{1 / 2}\right] \boldsymbol{Z}=0,
$$

and this clearly shows the equation (7.5) using the relation $\boldsymbol{W}\left[\varepsilon^{1 / 2}\right]=\mathcal{V}\left[\varepsilon^{1 / 2}\right] \boldsymbol{Z}\left[\varepsilon^{1 / 2}\right]$. Recall that the formal series $\Psi[\varepsilon]$ and $\Theta[\varepsilon]$ satisfy the equations (see the equations (5.14) and (5.16) of [15]),

$$
\left\{\begin{aligned}
(\mathscr{L}[\varepsilon], \mathscr{T}[\varepsilon]) \Psi[\varepsilon] & =0 \\
\left.(\Psi[\varepsilon]-\mathfrak{d}[\varepsilon])\right|_{R=0}+\left.\mathrm{V}[\varepsilon]\right|_{\Gamma_{0}} & =0
\end{aligned}\right.
$$

and

$$
\left\{\begin{aligned}
(\mathscr{L}[\varepsilon], \mathscr{T}[\varepsilon]) \Theta[\varepsilon] & =0 \\
\left.(\Theta[\varepsilon]+\mathfrak{h}[\varepsilon])\right|_{R=0}+\left.\mathrm{Q}[\varepsilon]\right|_{\Gamma_{0}} & =0
\end{aligned}\right.
$$

where the formal series $\mathfrak{d}[\varepsilon]$ is a formal series with operator coefficients taking values in the four dimensional space of rigid displacements on the semi-strip (see Theorem 5.3 of [15]). This formal series is related to $\mathrm{d}[\varepsilon]$ by the relations $\mathfrak{d}_{i}[\varepsilon]=\mathrm{d}_{i}[\varepsilon]$ for $i=1,2,3$ and $\mathfrak{d}_{n}[\varepsilon]=\varepsilon^{-1} \mathfrak{d}_{4}[\varepsilon]-b_{r}^{r} \mathfrak{d}_{1}[\varepsilon]-b_{r}^{s} \mathfrak{d}_{2}[\varepsilon]$ (see the equation (6.1) of [15]). A similar relation holds for $\mathfrak{h}[\varepsilon]$ and $h[\varepsilon]$.

By doing the change of variable $(r, s) \mapsto(T, s)$ in the equation (7.8), and using the definition of the operator formal series $\psi\left[\varepsilon^{1 / 2}\right]$, we find that:

$$
\left\{\begin{aligned}
&\left(\mathscr{L}\left[\varepsilon^{1 / 2}\right], \mathscr{T}\left[\varepsilon^{1 / 2}\right]\right) \psi\left[\varepsilon^{1 / 2}\right]=0 \\
&\left.\left(\psi\left[\varepsilon^{1 / 2}\right]-\mathfrak{c}\left[\varepsilon^{1 / 2}\right]\right)\right|_{R=0}+\left.\mathcal{V}\left[\varepsilon^{1 / 2}\right]\right|_{\Gamma_{0}}=0
\end{aligned}\right.
$$


where the formal series $\mathfrak{c}\left[\varepsilon^{1 / 2}\right]$ is obtained by doing the change of variable $(r, s) \mapsto$ $(T, s)$ in the coefficients of the formal series $\mathfrak{d}[\varepsilon]$.

The formal series $\mathfrak{c}\left[\varepsilon^{1 / 2}\right]$ is thus related to the formal series $\boldsymbol{d}\left[\varepsilon^{1 / 2}\right]$ in the same way as the formal series $\mathfrak{d}\left[\varepsilon^{1 / 2}\right]$ is related to the formal series $d\left[\varepsilon^{1 / 2}\right]$. In particular the equations

$$
\mathrm{d}\left[\varepsilon^{1 / 2}\right] \boldsymbol{\zeta}\left[\varepsilon^{1 / 2}\right]+\boldsymbol{d}\left[\varepsilon^{1 / 2}\right] \boldsymbol{Z}\left[\varepsilon^{1 / 2}\right]=\mathrm{h}\left[\varepsilon^{1 / 2}\right] \boldsymbol{f}\left[\varepsilon^{1 / 2}\right],
$$

and

$$
\mathfrak{d}\left[\varepsilon^{1 / 2}\right] \boldsymbol{\zeta}\left[\varepsilon^{1 / 2}\right]+\mathfrak{c}\left[\varepsilon^{1 / 2}\right] \boldsymbol{Z}\left[\varepsilon^{1 / 2}\right]=\mathfrak{h}\left[\varepsilon^{1 / 2}\right] \boldsymbol{f}\left[\varepsilon^{1 / 2}\right],
$$

are equivalent. This last equation is thus satisfied for the formal series $\zeta\left[\varepsilon^{1 / 2}\right]$ and $\boldsymbol{Z}\left[\varepsilon^{1 / 2}\right]$ solutions of Theorem 6.3.

Applying the equation (7.8) to the formal series $\zeta\left[\varepsilon^{1 / 2}\right]$, the equation (7.9) to the formal series $\boldsymbol{f}\left[\varepsilon^{1 / 2}\right]$ and the equation (7.10) to the formal series $\boldsymbol{Z}\left[\varepsilon^{1 / 2}\right]$, and summing the expressions so obtained, we find

$$
\left(\mathscr{L}\left[\varepsilon^{1 / 2}\right], \mathscr{T}\left[\varepsilon^{1 / 2}\right]\right) \boldsymbol{\Phi}\left[\varepsilon^{1 / 2}\right]=0,
$$

and this shows (7.6). Similarly we obtain

$$
\begin{aligned}
\left.\boldsymbol{\Phi}\left[\varepsilon^{1 / 2}\right]\right|_{R=0}+\left.\boldsymbol{v}\left[\varepsilon^{1 / 2}\right]\right|_{\Gamma_{0}}+ & \left.\boldsymbol{W}\left[\varepsilon^{1 / 2}\right]\right|_{\Gamma_{0}} \\
& -\mathfrak{d}\left[\varepsilon^{1 / 2}\right] \boldsymbol{\zeta}\left[\varepsilon^{1 / 2}\right]+\mathfrak{h}\left[\varepsilon^{1 / 2}\right] \boldsymbol{f}\left[\varepsilon^{1 / 2}\right]-\mathfrak{c}\left[\varepsilon^{1 / 2}\right] \boldsymbol{Z}\left[\varepsilon^{1 / 2}\right]=0,
\end{aligned}
$$

using the definition of $\boldsymbol{v}\left[\varepsilon^{1 / 2}\right]$ and $\boldsymbol{W}\left[\varepsilon^{1 / 2}\right]$. The equation (7.11) then shows (7.7). This ends the proof of the Theorem.

\section{C COMPARISON WITH THE KOITER MODEL}

We compare now the first terms of the formal series $\boldsymbol{v}\left[\varepsilon^{1 / 2}\right], \boldsymbol{W}\left[\varepsilon^{1 / 2}\right]$ and $\boldsymbol{\Phi}\left[\varepsilon^{1 / 2}\right]$ with the first terms in the expansion of the Koiter model.

Proposition 7.2 Let $\boldsymbol{v}\left[\varepsilon^{1 / 2}\right], \boldsymbol{W}\left[\varepsilon^{1 / 2}\right]$ and $\boldsymbol{\Phi}\left[\varepsilon^{1 / 2}\right]$ be the formal series defined in the previous subsection. The first terms of these series write

$$
\begin{aligned}
& \boldsymbol{v}\left[\varepsilon^{1 / 2}\right]=\boldsymbol{\zeta}^{0}+\varepsilon^{1 / 2} \boldsymbol{\zeta}^{1 / 2}+\mathcal{O}(\varepsilon), \\
& \boldsymbol{W}\left[\varepsilon^{1 / 2}\right]=\left\{\begin{array}{rlrl}
W_{T}\left[\varepsilon^{1 / 2}\right] & & \varepsilon^{1 / 2}\left(Z_{T}^{1 / 2}-X_{3} \partial_{T} Z_{3}^{0}\right) & +\mathcal{O}(\varepsilon), \\
W_{s}\left[\varepsilon^{1 / 2}\right] & = & \varepsilon^{1 / 2} Z_{s}^{1 / 2} & +\mathcal{O}(\varepsilon), \\
W_{3}\left[\varepsilon^{1 / 2}\right] & =Z_{3}^{0}+\varepsilon^{1 / 2} Z_{3}^{1 / 2} & +\mathcal{O}(\varepsilon),
\end{array}\right.
\end{aligned}
$$

and

$$
\boldsymbol{\Phi}\left[\varepsilon^{1 / 2}\right]=\varepsilon \boldsymbol{\Phi}^{1}+\mathcal{O}\left(\varepsilon^{3 / 2}\right) .
$$

Let $\zeta^{\prime}\left[\varepsilon^{1 / 2}\right]$ and $Z^{\prime}\left[\varepsilon^{1 / 2}\right]$ be the solution of the formal series problem (6.18). We have

$$
\boldsymbol{\zeta}^{0}=\boldsymbol{\zeta}^{\prime 0}, \quad \boldsymbol{\zeta}^{1 / 2}=\boldsymbol{\zeta}^{\prime 1 / 2}, \quad \boldsymbol{Z}^{0}=\boldsymbol{Z}^{\prime 0}, \quad Z_{T}^{1 / 2}=Z_{T}^{\prime 1 / 2} \quad \text { and } \quad Z_{s}^{1 / 2}=Z_{s}^{\prime 1 / 2},
$$

but $Z_{3}^{1 / 2} \neq Z_{3}^{\prime 1 / 2}$ in general. 
This Proposition is a consequence of Proposition 6.4 and the expression of the operators $\mathrm{V}^{k}$ in variables $\left(T, s, X_{3}\right)$. See [14] for further details.

\section{ESTIMATES FOR THE THREE-DIMENSIONAL ASYMPTOTICS}

We consider the shifted displacement $\boldsymbol{w}^{\varepsilon}$ solution of the equations (1.22). We denote by $\boldsymbol{w}(\varepsilon)$ the corresponding displacement in $\Omega$, solution of the equations (see (1.23)):

$$
(\mathrm{L}(\varepsilon), \mathrm{T}(\varepsilon)) \boldsymbol{w}(\varepsilon)=(-\boldsymbol{f}(\varepsilon), 0) \quad \text { in } \quad \Omega \times \Gamma_{ \pm} \quad \text { and } \quad \boldsymbol{w}(\varepsilon)=0 \quad \text { on } \quad \Gamma_{0} .
$$

\section{A AnSATZ OF 3D EXPANSION}

With the formal series defined in the previous section, we set for all $N \in \mathbb{N}$,

$$
\begin{aligned}
& \boldsymbol{w}^{[N]}(\varepsilon)\left(x_{1}, x_{2}, X_{3}\right):= \\
& \sum_{k=0}^{N} \varepsilon^{k / 2}\left(\boldsymbol{v}^{k / 2}\left(x_{1}, x_{2}, X_{3}\right)+\chi(r) \boldsymbol{W}^{k / 2}\left(\varepsilon^{-1 / 2} r, s, X_{3}\right)+\chi(r) \boldsymbol{\Phi}^{k / 2}\left(\varepsilon^{-1} r, s, X_{3}\right)\right) .
\end{aligned}
$$

This defines an element of $\mathrm{H}^{1}(\Omega)^{3}$. We write it

$$
\boldsymbol{w}^{[N]}(\varepsilon)=\boldsymbol{v}^{[N]}(\varepsilon)+\chi(r) \boldsymbol{W}^{[N]}(\varepsilon)+\chi(r) \boldsymbol{\Phi}^{[N]}(\varepsilon),
$$

with evident notations. Using (7.7) we see that

$$
\left.\forall N \in \mathbb{N} \quad \boldsymbol{w}^{[N]}(\varepsilon)\right|_{\Gamma_{0}}=0, \quad \text { or } \quad \boldsymbol{w}^{[N]}(\varepsilon) \in V(\Omega),
$$

where $V(\Omega)=\left\{\boldsymbol{u} \in \mathrm{H}^{1}(\Omega)^{3}|\boldsymbol{u}|_{\Gamma_{0}}=0\right\}$ is the variational space associated with the 3D problem. We set $\tilde{a}_{3 \mathrm{D}}^{\varepsilon}$ the bilinear form defined by the formula

$$
\tilde{a}_{3 \mathrm{D}}^{\varepsilon}(\boldsymbol{w}, \boldsymbol{v}):=\int_{\Omega^{\varepsilon}} A^{i j k \ell} \tilde{e}_{k \ell}(\boldsymbol{w}) \tilde{e}_{i j}(\boldsymbol{v}) \mathrm{d} V
$$

where $\tilde{e}_{i j}(\boldsymbol{v})=e_{i j}\left(\boldsymbol{\mu}^{-1}\left(x_{3}\right) \boldsymbol{v}\right)$ with $\boldsymbol{\mu}\left(x_{3}\right)$ the shifter defined in (1.21). This bilinear form corresponds to the energy (1.19) for shifted displacements. We denote by $\tilde{a}_{3 \mathrm{D}}(\varepsilon)$ the corresponding bilinear form on $V(\Omega)$.

We have the following Korn estimate (see section 4 of [7]):

$$
\forall \boldsymbol{v} \in V(\Omega), \quad\|\boldsymbol{v}\|_{\mathrm{H}^{1}(\Omega)^{3}} \leq \varepsilon^{-1} C\left\|e_{i j}\left(x_{\alpha}, \varepsilon X_{3} ; \mathrm{D}_{\alpha}, \varepsilon^{-1} \partial_{X_{3}}\right)(\boldsymbol{v})\right\|_{\mathrm{L}^{2}(\Omega)},
$$

where $C$ is independent on $\varepsilon$. Moreover, the operator $\boldsymbol{\mu}(\varepsilon)$ acts on $V(\Omega)$ and is invertible with bounded inverse in $\varepsilon$ : We have

$$
\forall \boldsymbol{v} \in \mathrm{H}^{1}(\Omega), \quad c\|\boldsymbol{v}\|_{\mathrm{H}^{1}(\Omega)^{3}} \leq\|\boldsymbol{\mu}(\varepsilon) \boldsymbol{v}\|_{\mathrm{H}^{1}(\Omega)^{3}} \leq C\|\boldsymbol{v}\|_{\mathrm{H}^{1}(\Omega)^{3}},
$$

where $c$ and $C$ are constants independent on $\varepsilon$. Using (8.5) and (8.6) we find

$$
\forall \boldsymbol{v} \in V(\Omega), \quad\|\boldsymbol{v}\|_{\mathrm{H}^{1}(\Omega)^{3}}^{2} \leq \varepsilon^{-2} C_{0} \tilde{a}_{3 \mathrm{D}}(\varepsilon)(\boldsymbol{v}, \boldsymbol{v}),
$$

where $C_{0}$ is independent on $\varepsilon$. 


\section{B VAlidation}

Similarly to the section 4 and Proposition 5.1, we first give the rough estimate:

Lemma 8.1 With the notations of the previous section, we have

$$
\left\|\boldsymbol{w}(\varepsilon)-\boldsymbol{w}^{[N]}(\varepsilon)\right\|_{\mathrm{H}^{1}(\Omega)^{3}} \leq C \varepsilon^{N / 2-4} .
$$

We do not give the proof of this lemma, as it is very close to the proof of Proposition 5.1 and very technical (see $[10,14]$ for similar calculations). We use the formal series equations satisfied by the formal series and the Korn inequality to conclude.

Let $\boldsymbol{v}\left[\varepsilon^{1 / 2}\right], \boldsymbol{W}\left[\varepsilon^{1 / 2}\right]$ and $\boldsymbol{\Phi}\left[\varepsilon^{1 / 2}\right]$ be the formal series defined in the previous subsections. We easily see that

$$
\forall k \geq 0, \quad\left\|\boldsymbol{v}^{k / 2}\right\|_{\mathrm{H}^{1}(\Omega)} \leq C
$$

Similarly, using the exponential decay of the boundary layer terms, we have

$$
\begin{aligned}
& \left\|\chi \boldsymbol{W}^{k / 2}\right\|_{\mathrm{L}^{2}(\Omega)} \leq C \varepsilon^{1 / 4}, \quad \text { and } \quad\left\|\chi \boldsymbol{\Phi}^{k / 2}\right\|_{\mathrm{L}^{2}(\Omega)} \leq C \varepsilon^{1 / 2}, \\
& \left\|\chi \boldsymbol{W}^{k / 2}\right\|_{\mathrm{H}^{1}(\Omega)} \leq C \varepsilon^{-1 / 4}, \quad \text { and } \quad\left\|\chi \boldsymbol{\Phi}^{k / 2}\right\|_{\mathrm{H}^{1}(\Omega)} \leq C \varepsilon^{-1 / 2} \text {, }
\end{aligned}
$$

where the constants $C$ are independent on $\varepsilon$. Using the previous lemma, we get the following result:

Theorem 8.2 Let $\boldsymbol{w}(\varepsilon)$ be the shifted displacement solution of the three-dimensional equations, and let $\boldsymbol{w}^{[N]}(\varepsilon)$ be the 1-form field defined in (8.3). Then we have:

$$
\forall N \geq 0, \quad\left\|\boldsymbol{w}(\varepsilon)-\boldsymbol{w}^{[N]}(\varepsilon)\right\|_{\mathrm{H}^{1}(\Omega)^{3}} \leq C \varepsilon^{N / 2} .
$$

Proof. Let $N \geq 0$. Using the estimate (8.8), we get

$$
\left\|\boldsymbol{w}(\varepsilon)-\boldsymbol{w}^{[N+10]}(\varepsilon)\right\|_{\mathrm{H}^{1}(\Omega)^{3}} \leq C \varepsilon^{N / 2+1},
$$

whence

$$
\begin{aligned}
\| \boldsymbol{w}(\varepsilon) & -\boldsymbol{w}^{[N]}(\varepsilon) \|_{\mathrm{H}^{1}(\Omega)^{3}} \\
& \leq \sum_{k=N+1}^{N+10} \varepsilon^{k / 2}\left(\left\|\boldsymbol{v}^{k / 2}\right\|_{\mathrm{H}^{1}(\Omega)^{3}}+\left\|\chi \boldsymbol{W}^{k / 2}\right\|_{\mathrm{H}^{1}(\Omega)^{3}}+\left\|\chi \boldsymbol{\Phi}^{k / 2}\right\|_{\mathrm{H}^{1}(\Omega)^{3}}\right)+C \varepsilon^{N / 2+1} .
\end{aligned}
$$

The previous estimates of the terms in $\varepsilon$ yields the result. by

Recall that the 3D displacement $\boldsymbol{u}(\varepsilon)$ and the shifted displacement $\boldsymbol{w}(\varepsilon)$ are related

$$
u_{\sigma}(\varepsilon)=w_{\sigma}(\varepsilon)-\varepsilon X_{3} b_{\sigma}^{\alpha}\left(x_{1}, x_{2}\right) w_{\alpha}(\varepsilon) \quad \text { and } \quad u_{3}(\varepsilon)=w_{3}(\varepsilon) .
$$


We deduce that we have a similar asymptotic expansions for the 3D displacement. Moreover, as the shifter differs from the identity by a term of order $\varepsilon$ of order of derivation zero, we deduce that the terms of order 0 and 1 in $\varepsilon^{1 / 2}$ are equals for $\boldsymbol{w}(\varepsilon)$ and $\boldsymbol{u}(\varepsilon)$. Thus the proposition 7.2 is the same for these displacements.

Note eventually that using Lemma 8.1 written in $\Omega^{\varepsilon}$, and using estimates of the terms of the expansion in physical variables, we obtain the multiscale expansion for the shifted displacement in $\Omega^{\varepsilon}$. This shows Theorem 1.2.

Finally, using Theorem 8.2 and Proposition and 7.2, we easily show Proposition 1.3.

\section{Appendix A: Proof of lemma 6.5}

1. We first compare the operators $\breve{\mathcal{A}}^{1 / 2}$ and $\breve{\mathcal{K}}^{1 / 2}$. We have the following estimate (see Proposition 4.2 of [15]):

$$
\operatorname{deg} \mathrm{A}^{3} \leq\left(\begin{array}{ll}
4 & 5 \\
5 & 4
\end{array}\right)
$$

Moreover, the operators $\mathrm{A}^{k}$ are of order at most $k+2$ for $k \geq 4$.

Using the definition (6.6) of the formal series $\breve{\mathcal{A}}\left[\varepsilon^{1 / 2}\right]$, we have, similarly to (6.3):

$$
\breve{\mathcal{A}}\left[\varepsilon^{1 / 2}\right]=\sum_{k \geq 0} \varepsilon^{k} \breve{\mathcal{A}}^{(k)}\left[\varepsilon^{1 / 2}\right]
$$

with

$$
\breve{\mathcal{A}}^{(k)}\left[\varepsilon^{1 / 2}\right] \breve{\boldsymbol{Z}}\left[\varepsilon^{1 / 2}\right]=\left(\varepsilon \mathcal{A}_{\sigma}^{(k)}\left[\varepsilon^{1 / 2}\right] \boldsymbol{Z}\left[\varepsilon^{1 / 2}\right], \varepsilon^{1 / 2} \mathcal{A}_{3}^{(k)}\left[\varepsilon^{1 / 2}\right] \boldsymbol{Z}\left[\varepsilon^{1 / 2}\right]\right),
$$

where the formal series $\boldsymbol{Z}\left[\varepsilon^{1 / 2}\right]$ and $\breve{Z}\left[\varepsilon^{1 / 2}\right]$ are related by (3.8). Using (8.14) and (6.2), we see that we have

$$
\forall k \geq 0, \quad \breve{\mathcal{A}}^{(k)}\left[\varepsilon^{1 / 2}\right]=\left(\sum_{\ell \geq-(k+1)} \varepsilon^{\ell / 2} \breve{\mathcal{A}}_{\sigma}^{k, \ell / 2}, \sum_{\ell \geq-(k+2)} \varepsilon^{\ell / 2} \breve{\mathcal{A}}_{3}^{k, \ell / 2}\right) .
$$

Using (8.13) we see that

$$
\breve{\mathcal{A}}_{\sigma}\left[\varepsilon^{1 / 2}\right]=\sum_{k \geq 0} \sum_{\ell \geq 0} \varepsilon^{(2 k+\ell-k-1) / 2} \breve{\mathcal{A}}_{\sigma}^{k,(\ell-k-1) / 2}=\sum_{n \geq-1} \varepsilon^{n / 2} \sum_{k=0}^{n+1} \breve{\mathcal{A}}_{\sigma}^{k,(n-2 k) / 2},
$$

where we used the fact that $\breve{\mathcal{A}}_{\sigma}^{k, \ell / 2}=0$ for $\ell \leq-(k+1)$. Similarly, for the transverse component we see that

$$
\breve{\mathcal{A}}_{3}\left[\varepsilon^{1 / 2}\right]=\sum_{n \geq-2} \varepsilon^{n / 2} \sum_{k=0}^{n+2} \breve{\mathcal{A}}_{3}^{k,(n-2 k) / 2} .
$$


In particular, we have that

$$
\breve{\mathcal{A}}_{\sigma}^{1 / 2}=\breve{\mathcal{A}}_{\sigma}^{0,1 / 2}+\breve{\mathcal{A}}_{\sigma}^{1,-1 / 2}+\breve{\mathcal{A}}_{\sigma}^{2,-3 / 2} \text { and } \breve{\mathcal{A}}_{3}^{1 / 2}=\breve{\mathcal{A}}_{3}^{0,1 / 2}+\breve{\mathcal{A}}_{3}^{1,-1 / 2}+\breve{\mathcal{A}}_{3}^{2,-3 / 2}+\breve{\mathcal{A}}_{3}^{3,-5 / 2} .
$$

Similarly, for the computation of the operator $\breve{\mathcal{K}}^{1 / 2}$, we have

$$
\breve{\mathcal{K}}_{\sigma}^{1 / 2}=\breve{\mathcal{M}}_{\sigma}^{1 / 2}+\breve{\mathcal{B}}_{\sigma}^{-3 / 2} \text { and } \quad \breve{\mathcal{K}}_{3}^{1 / 2}=\breve{\mathcal{M}}_{3}^{1 / 2}+\breve{\mathcal{B}}_{3}^{-3 / 2}
$$

where the operators $\breve{\mathcal{M}}^{k / 2}$ and $\breve{\mathcal{B}}^{k / 2}$ are defined as before from the operators $\mathrm{M}$ and $\mathrm{B}$. The equations (8.15) shows that for $k \geq 4$, the operators $\mathrm{A}^{k}$ play no role in the computation of the operator $\breve{\mathcal{A}}^{1 / 2}$.

As $A_{3}^{3}$ is only of order 4 in $z_{3}$ (see (8.12)), we have $\breve{\mathcal{A}}_{3}^{3,-5 / 2}=0$. The operator $\mathrm{A}^{1}$ being the zero operator, we have $\breve{\mathcal{A}}_{\sigma}^{1,-1 / 2}=\breve{\mathcal{A}}_{3}^{1,-1 / 2}=0$. As $\mathrm{A}^{0}$ is the membrane operator, it is clear that $\breve{\mathcal{A}}_{\sigma}^{0,1 / 2}=\breve{\mathcal{M}}_{\sigma}^{1 / 2}$ and $\breve{\mathcal{A}}_{3}^{0,1 / 2}=\breve{\mathcal{M}}_{3}^{1 / 2}$.

Now using the expression of the operators $A^{2}$ (see Theorem 4.4 of [15]) and $B^{2}$, we compute directly that $\breve{\mathcal{A}}_{\sigma}^{2,-3 / 2}=\breve{\mathcal{B}}_{\sigma}^{-3 / 2}=0$ and

$$
\breve{\mathcal{A}}_{3}^{2,-3 / 2} \breve{\boldsymbol{Z}}=\breve{\mathcal{B}}_{3}^{-3 / 2} \breve{\boldsymbol{Z}}=\frac{2}{3} \widetilde{\lambda} \Gamma_{s s}^{r}(0, s) \partial_{T T T} \breve{Z}_{3}
$$

This eventually shows that $\breve{\mathcal{K}}^{1 / 2}=\breve{\mathcal{A}}^{1 / 2}$.

2. We now compute the operator $\breve{d}_{3}^{0}$ or equivalently the operator $\breve{\mathcal{D}}_{3}^{0}$ (see (6.14)). We have that

$$
\breve{\mathcal{D}}\left[\varepsilon^{1 / 2}\right]=\sum_{k \geq 0} \varepsilon^{k} \breve{\mathcal{D}}^{(k)}\left[\varepsilon^{1 / 2}\right]
$$

where the formal series $\breve{\mathcal{D}}^{(k)}\left[\varepsilon^{1 / 2}\right]$ are associated with the formal series $\mathcal{D}^{(k)}\left[\varepsilon^{1 / 2}\right]$ by the formula $\breve{\mathcal{D}}^{(k)}\left[\varepsilon^{1 / 2}\right] \breve{\boldsymbol{Z}}\left[\varepsilon^{1 / 2}\right]=\mathcal{D}^{(k)}\left[\varepsilon^{1 / 2}\right] \boldsymbol{Z}\left[\varepsilon^{1 / 2}\right]$.

As the operators $D_{3}^{k}$ are of order at most $k$ in $r$, we have as in Lemma 6.1:

$$
\breve{\mathcal{D}}_{3}\left[\varepsilon^{1 / 2}\right]=\sum_{n \geq-1} \varepsilon^{n / 2} \breve{\mathcal{D}}_{3}^{n / 2} \quad \text { with } \quad \breve{\mathcal{D}}_{3}^{n / 2}=\sum_{k=0}^{n+1} \breve{\mathcal{D}}_{3}^{k,(n-2 k) / 2} .
$$

We thus deduce that

$$
\breve{\mathcal{D}}_{3}^{0}=\breve{\mathcal{D}}_{3}^{0,0}+\breve{\mathcal{D}}_{3}^{1,-1 / 2} .
$$

As $D_{3}^{1}$ is the zero operator, we deduce that $\breve{\mathcal{D}}_{3}^{1,-1 / 2}=0$. Moreover as $D_{3}^{0} \boldsymbol{z}=z_{3}$, we have that $\breve{\mathcal{D}}_{3}^{0,0}=0$. This shows that $\breve{d}_{3}^{0} z=z_{3}$.

3. We compute now the operator $\breve{\mathcal{D}}_{n}^{-1 / 2}$. As the operators $D_{n}^{k}$ are of degree of derivative at most $k+1$ in $r$, we have with evident notations:

$$
\breve{\mathcal{D}}_{n}\left[\varepsilon^{1 / 2}\right]=\sum_{\ell \geq-2} \varepsilon^{\ell / 2} \breve{\mathcal{D}}_{3}^{\ell / 2} \quad \text { with } \quad \breve{\mathcal{D}}_{n}^{\ell / 2}=\sum_{k=0}^{\ell+2} \breve{\mathcal{D}}_{3}^{k,(\ell-2 k) / 2}
$$


We deduce that

$$
\breve{\mathcal{D}}_{n}^{-1 / 2}=\breve{\mathcal{D}}_{n}^{0,-1 / 2}+\breve{\mathcal{D}}_{n}^{1,-3 / 2} .
$$

But it is clear that $\breve{\mathcal{D}}_{n}^{0,-1 / 2}=0$, using $D_{n}^{0} \boldsymbol{z}=\partial_{r} z_{3}$. To compute the operator $\breve{\mathcal{D}}_{n}^{1,-3 / 2}$, we only need to know the terms with 2 derivatives in $r$ acting on $z_{3}$ in the expression of the operator $\mathfrak{d}_{n}^{2}$ (see Theorem 5.3 in [15]).

We will show later the following equation, for $z \in \Sigma\left(S_{0}\right)$ :

$$
\mathfrak{d}_{n}^{2} \boldsymbol{z}=\left.\left(c_{2} \partial_{r r} z_{3}+P \boldsymbol{z}\right)\right|_{\partial S_{0}},
$$

where $c_{2}$ is a non zero constant depending only on $\lambda$ and $\mu$, and $P$ is an operator taking values in $\mathscr{C}^{\infty}\left(S_{0}\right)$ such that in coordinates $(r, s)$, we have

$$
P \boldsymbol{z}=P^{r} z_{r}+P^{s} z_{s}+P^{3} z_{3},
$$

where $P^{r}$ and $P^{s}$ are operators of order 2 acting on $z_{r}$ and $z_{s}$ respectively, and where

$$
P^{3} z_{3}=p_{1}^{3}(r, s) \partial_{r s} z_{3}+p_{2}^{3}(r, s) \partial_{s s} z_{3}+Q^{3} z_{3},
$$

with $Q^{3}$ and operator of order 1 in $z_{3}$ and $p_{1}^{3}, p_{2}^{3}$ functions of the variables $(r, s)$.

The equation (8.17) shows that

$$
\breve{\mathcal{D}}_{n}^{(1)}\left[\varepsilon^{1 / 2}\right](\breve{\boldsymbol{Z}})=\varepsilon^{-3 / 2} c_{2} \partial_{T T} \breve{Z}_{3}+\sum_{\ell \geq-2} \varepsilon^{\ell / 2} \breve{\mathcal{D}}_{n}^{1, \ell / 2}(\breve{\boldsymbol{Z}}) .
$$

We deduce that

$$
\breve{\mathcal{D}}_{n}^{1,-3 / 2}(\breve{\boldsymbol{Z}})=c_{2} \partial_{T T} \breve{Z}_{3}
$$

and this yields the result.

Proof of (8.17). Using the proof of Theorem 5.3 of [15], the operators $\Psi^{2}$ and $\mathfrak{d}^{2}$ are such that if $\boldsymbol{z} \in \Sigma\left(S_{0}\right), \boldsymbol{\varphi}:=\Psi^{2} \boldsymbol{z}-\mathfrak{d}^{2} \boldsymbol{z}$ is the unique solution of the problem

$$
\left\{\begin{aligned}
\mathscr{L}^{0} \boldsymbol{\varphi} & =-\mathscr{L}^{1} \Psi^{1} \boldsymbol{z} \text { in } \partial S_{0} \times \Sigma^{+}, \\
\mathscr{T}^{0} \boldsymbol{\varphi} & =-\mathscr{T}^{1} \Psi^{1} \boldsymbol{z} \text { on } \partial S_{0} \times \gamma_{+} \times \gamma_{-}, \\
\left.\boldsymbol{\varphi}\right|_{R=0}+\left.\mathrm{V}^{2} \boldsymbol{z}\right|_{\Gamma_{0}} & =0
\end{aligned}\right.
$$

with $\Psi^{2} \boldsymbol{z} \in \mathscr{C}^{\infty}\left(\partial S_{0}, \mathfrak{H}\left(\Sigma^{+}\right)\right)$(see the beginning of Section 6 ) and $\mathfrak{d}^{2} \boldsymbol{z}$ in the space spanned by the 4 rigid displacement in coordinates $\left(R, s, X_{3}\right)$ (see the equation (5.8) of [15]). Here, $\gamma_{ \pm}$denote the two upper and lower lines $\left\{X_{3}= \pm 1\right\}$ of $\Sigma^{+}$. The operator $\mathrm{V}^{2}$ writes (see [15]):

$$
\mathrm{V}_{\sigma}^{2}(\boldsymbol{z})=\frac{X_{3}^{2}}{2} p \mathrm{D}_{\sigma} \gamma_{\alpha}^{\alpha}(\boldsymbol{z}) \quad \text { and } \quad \mathrm{V}_{3}^{2}(\boldsymbol{z})=\frac{X_{3}^{2}}{2} p\left(\rho_{\alpha}^{\alpha}(\boldsymbol{z})-p b_{\alpha}^{\alpha} \gamma_{\beta}^{\beta}(\boldsymbol{z})-2 b_{\alpha}^{\beta} \gamma_{\beta}^{\alpha}(\boldsymbol{z})\right)
$$

As in the proof of Theorem 5.3 of [15] we split the solution $\varphi$ of the previous system into the solution of

$$
\left\{\begin{aligned}
\mathscr{L}^{0} \boldsymbol{\phi} & =-\mathscr{L}^{1} \Psi^{1} \boldsymbol{z} \text { in } \partial S_{0} \times \Sigma^{+} \\
\mathscr{T}^{0} \boldsymbol{\phi} & =-\mathscr{T}^{1} \Psi^{1} \boldsymbol{z} \text { on } \partial S_{0} \times \gamma_{+} \times \gamma_{-} \\
\left.\boldsymbol{\phi}\right|_{R=0} & =0
\end{aligned}\right.
$$


and the solution of the system

$$
\left\{\begin{aligned}
\mathscr{L}^{0} \boldsymbol{\phi} & =0 \text { in } \partial S_{0} \times \Sigma^{+}, \\
\mathscr{T}^{0} \boldsymbol{\phi} & =0 \text { in } \partial S_{0} \times \gamma_{+} \times \gamma_{-}, \\
\left.\boldsymbol{\phi}\right|_{R=0}+\left.\mathrm{V}^{2} \boldsymbol{z}\right|_{\Gamma_{0}} & =0
\end{aligned}\right.
$$

Using the expression of the operator $\Psi^{1}$ (see the equation (5.13) of [15]), we see that the right-hand side of (8.18) is a linear combination of elements of $\mathscr{C}^{\infty}\left(\partial S_{0}, \mathfrak{K}\left(\Sigma^{+}\right)\right)$whose coefficients are traces on $\partial S_{0}$ of operators acting on $\Sigma\left(S_{0}\right)$ with degrees of derivative at most equal to 2 in $z_{\sigma}$ and 1 in $z_{3}$. Thus the corresponding parts of the operators $\Psi^{2}$ and $\mathfrak{d}^{2}$ are only involved in the definition of the operator $P$ of the equation (8.17).

Similarly the solution of (8.19) splits into the sum of the solutions of systems with only one single non vanishing component in the right-hand side, polynomial in $X_{3}$. The expression of $\mathrm{V}^{2}$ show that in the coordinate system $(r, s)$, only $V_{3}^{2} \boldsymbol{z}$ contains derivatives of order 2 in $z_{3}$. The components $V_{r}^{2} \boldsymbol{z}$ and $V_{s}^{2} \boldsymbol{z}$ only enter into the expression of $P$. In the coordinate system $(r, s)$, we have

$$
V_{3}^{2} \boldsymbol{z}=\frac{X_{3}^{2}}{2} p \partial_{r r} z_{3}+\mathrm{Op}(\boldsymbol{z})
$$

where $\operatorname{Op}(\boldsymbol{z})$ denotes an operator of order 2 with derivative of order 1 in $r$ on $z_{3}$. We seek the solution of the system of the form (8.19) with a polynomial right-hand side, and we use the splitting of the operator $\left(\mathscr{L}^{0}, \mathscr{T}^{0}\right)$ into the operators $\left(\mathscr{L}_{s}^{0}, \mathscr{T}_{s}^{0}\right)$ and $\left(\mathscr{L}_{R}^{0}, \mathscr{L}_{3}^{0}, \mathscr{T}_{R}^{0}, \mathscr{T}_{3}^{0}\right.$ ) (see the equation (5.6) of [15]). We show (see section 6.1 of [12]) that there exist $\left(\bar{\varphi}_{R}^{2}, \bar{\varphi}_{3}^{2}\right) \in \mathfrak{H}\left(\Sigma^{+}\right)^{2}$ and two constants $c$ and $c^{\prime}$ such that

$$
\left\{\begin{aligned}
\left(\mathscr{L}_{R}^{0}, \mathscr{L}_{3}^{0}\right)\left(\bar{\varphi}_{R}^{2}, \bar{\varphi}_{3}^{2}\right) & =0 \text { in } \Sigma^{+}, \\
\left(\mathscr{T}_{R}^{0}, \mathscr{T}_{3}^{0}\right)\left(\bar{\varphi}_{R}^{2}, \bar{\varphi}_{3}^{2}\right) & =0 \text { on } \gamma_{+} \times \gamma_{-}, \\
\left.\bar{\varphi}_{R}^{1}\right|_{R=0}+c X_{3} & =0 \\
\left.\bar{\varphi}_{3}^{1}\right|_{R=0}-c^{\prime} & =X_{3}^{2} .
\end{aligned}\right.
$$

The coefficients $c$ and $c^{\prime}$ are the coefficients of the rigid displacements $\mathcal{Z}^{4}$ et $\mathcal{Z}^{3}$ (see [12] and the equation (5.8) of [15]). For parity reasons in $X_{3}$, the coefficients of $\mathcal{Z}^{1}$ is zero, and the displacement $\mathcal{Z}^{2}$ is not involved. We can show that the coefficient $c^{\prime}$ is non zero (see lemma 6.1 in [12]).

We construct the solution $\phi$ of (8.19) depending on the term $p \partial_{r r} z_{3}$ in the operator $\mathrm{V}^{2}$ by multiplying the solution of (8.20) with $-\frac{1}{2} p \partial_{r r} z_{3}$. We thus deduce that $\mathfrak{d}_{n}^{4} \boldsymbol{z}=$ $-\frac{1}{2} c^{\prime} p \partial_{r r} z_{3}+P \boldsymbol{z}$, where $P$ satisfies the correct order conditions. We get the result by setting $c_{2}=-\frac{1}{2} c^{\prime} p \neq 0$.

\section{Appendix B: Proof of Theorem 1.4}

1. We first compute the energy norm of $\boldsymbol{u}^{\varepsilon}$. We define the energy for the shifted 
displacement:

$$
\tilde{E}_{3 \mathrm{D}}^{\varepsilon}[\boldsymbol{w}]=\int_{\Omega^{\varepsilon}} A^{i j k \ell} \tilde{e}_{i j}(\boldsymbol{w}) \tilde{e}_{k \ell}(\boldsymbol{w}) \mathrm{d} V,
$$

where $\tilde{e}_{i j}(\boldsymbol{w})$ is defined as $\tilde{e}_{i j}(\boldsymbol{w})=e_{i j}\left(\boldsymbol{\mu}^{-1}\left(x_{3}\right) \boldsymbol{w}\right)$ is the strain tensor associated with the shifted displacement. We have $\tilde{E}_{3 \mathrm{D}}^{\varepsilon}\left[\boldsymbol{w}^{\varepsilon}\right]=E_{3 \mathrm{D}}^{\varepsilon}\left[\boldsymbol{u}^{\varepsilon}\right]$. The expansion of the operator $\tilde{e}_{i j}$ on $\Omega^{\varepsilon}$ is given in Proposition 3.2 of [15].

Using the positivity of the rigidity tensor, we have that

$$
c\left\|\tilde{e}_{i j}\left(\boldsymbol{w}^{\varepsilon}\right)\right\|_{\mathrm{L}^{2}\left(\Omega^{\varepsilon}\right)}^{2} \leq \tilde{E}_{3 \mathrm{D}}^{\varepsilon}\left[\boldsymbol{w}^{\varepsilon}\right] \leq C\left\|\tilde{e}_{i j}\left(\boldsymbol{w}^{\varepsilon}\right)\right\|_{\mathrm{L}^{2}\left(\Omega^{\varepsilon}\right)}^{2},
$$

for constants $c$ and $C$ independent on $\varepsilon$.

Recall (see Theorem 4.4 of [15]), that the operators $V^{1}$ and $V^{2}$ of the formal series $\mathrm{V}[\varepsilon]$ write

$$
\mathrm{V}^{1} z=\left\{\begin{array}{l}
-X_{3} \theta_{\sigma}(\boldsymbol{z}) \\
-X_{3} p \gamma_{\alpha}^{\alpha}(\boldsymbol{z})
\end{array} \quad \text { and } \quad \mathrm{V}^{2} z=\left\{\begin{array}{l}
\frac{X_{3}^{2}}{2} \mathrm{D}_{\sigma} \gamma_{\alpha}^{\alpha}(\boldsymbol{z}) \\
\frac{X_{3}^{2}}{2} p\left(\rho_{\alpha}^{\alpha}(\boldsymbol{z})-p b_{\alpha}^{\alpha} \gamma_{\beta}^{\beta}(\boldsymbol{z})-2 b_{\alpha}^{\beta} \gamma_{\beta}^{\alpha}(\boldsymbol{z})\right)
\end{array}\right.\right.
$$

where $p=\lambda(\lambda+2 \mu)^{-1}$. The following lemma precise the first part of Proposition 7.2.

Lemma 8.3 With the notations of Proposition 7.2, we have

$$
\boldsymbol{v}^{1}=\boldsymbol{\zeta}^{1}+\mathrm{V}^{1} \boldsymbol{\zeta}^{0}=\left\{\begin{array}{l}
\zeta_{\sigma}^{1}-X_{3}\left(\mathrm{D}_{\sigma} \zeta_{3}^{0}+b_{\sigma}^{\alpha} \zeta_{\alpha}^{0}\right) \\
\zeta_{3}^{1}-X_{3} p \gamma_{\alpha}^{\alpha}\left(\boldsymbol{\zeta}^{0}\right)
\end{array}\right.
$$

and in the coordinate system $\left(T, s, X_{3}\right)$,

$$
\boldsymbol{W}^{1}=\left(\begin{array}{lll}
Z_{T}^{1} & -X_{3} \partial_{T} Z_{3}^{1 / 2} \\
Z_{s}^{1} & -X_{3} \partial_{s} Z_{3}^{0} \\
Z_{3}^{1} & -X_{3} p \partial_{T} Z_{T}^{1 / 2}+X_{3} p b_{\alpha}^{\alpha}(0, s) Z_{3}^{0} & +\frac{X_{3}^{2}}{2} p \partial_{T T} Z_{3}^{0}
\end{array}\right) .
$$

Proof of Lemma 8.3. The equation (8.23) is clear using the definition of $\boldsymbol{v}\left[\varepsilon^{1 / 2}\right]$ and the expression of $\mathrm{V}^{1}$.

Now using the formal series (7.2), we have

$$
\boldsymbol{W}^{1}=\mathcal{V}^{0} \boldsymbol{Z}^{1}+\mathcal{V}^{1 / 2} \boldsymbol{Z}^{1 / 2}+\mathcal{V}^{1} \boldsymbol{Z}^{0}
$$

with

$$
\mathcal{V}^{0}=\mathcal{V}^{0,0}, \quad \mathcal{V}^{1 / 2}=\mathcal{V}^{0,1 / 2}+\mathcal{V}^{1,-1 / 2} \quad \text { and } \quad \mathcal{V}^{1}=\mathcal{V}^{0,1}+\mathcal{V}^{1,0}+\mathcal{V}^{2,-1}
$$

But using the definition of the operators $\mathcal{V}^{k, n}$, we easily see that $\mathcal{V}^{0}=\mathcal{V}^{0,0}$ is the identity operator. Similarly, using the expression of $\mathrm{V}^{1}$ and $\mathrm{V}^{2}$ we see that

$$
\mathcal{V}^{1,-1 / 2} \boldsymbol{Z}=\left\{\begin{array}{l}
-X_{3} \partial_{T} Z_{3}, \\
0, \\
-X_{3} p \partial_{T} Z_{T},
\end{array} \quad \mathcal{V}^{2,-1} \boldsymbol{Z}=\left\{\begin{array}{l}
\frac{X_{3}^{2}}{2} p \partial_{T T} Z_{T}, \\
0, \\
\frac{X_{3}^{2}}{2} p \partial_{T T} Z_{3}
\end{array}\right.\right.
$$


and

$$
\mathcal{V}^{1,0} \boldsymbol{Z}=\left\{\begin{array}{l}
-X_{3}\left(b_{r}^{r}(0, s) Z_{T}+b_{r}^{s}(0, s) Z_{s}\right), \\
-X_{3}\left(\partial_{s} Z_{3}+b_{s}^{r}(0, s) Z_{T}+b_{s}^{s}(0, s) Z_{s}\right), \\
-X_{3} p\left(\partial_{s} Z_{s}+\Gamma_{\alpha r}^{\alpha}(0, s) Z_{T}+\Gamma_{\alpha s}^{\alpha}(0, s) Z_{s}-b_{\alpha}^{\alpha}(0, s) Z_{3}\right),
\end{array}\right.
$$

and this shows the result.

We now give estimates corresponding to (8.9) on the physical shell.

Lemma 8.4 Suppose that $\boldsymbol{\zeta} \in \Sigma\left(S_{0}\right)$ and $\boldsymbol{Z} \in \mathscr{C}^{\infty}\left(\partial S_{0}, \mathfrak{T}\left(\mathbb{R}^{+}\right)\right.$are generic, independent of $\varepsilon$, non zero $2 D$ displacement and $2 D$ boundary layer term respectively. Then we have

$$
\begin{gathered}
\|\boldsymbol{\zeta}\|_{\mathrm{L}^{2}\left(\Omega^{\varepsilon}\right)}=\mathcal{O}\left(\varepsilon^{1 / 2}\right), \quad\|\boldsymbol{Z}\|_{\mathrm{L}^{2}\left(\Omega^{\varepsilon}\right)}=\mathcal{O}\left(\varepsilon^{3 / 4}\right), \\
\left\|\partial_{\alpha} \boldsymbol{\zeta}\right\|_{\mathrm{L}^{2}\left(\Omega^{\varepsilon}\right)}=\mathcal{O}\left(\varepsilon^{1 / 2}\right), \quad \text { and } \quad\left\|\partial_{\alpha} \boldsymbol{Z}\right\|_{\mathrm{L}^{2}\left(\Omega^{\varepsilon}\right)}=\mathcal{O}\left(\varepsilon^{1 / 4}\right) .
\end{gathered}
$$

Let $\boldsymbol{v} \in \mathscr{C}^{\infty}\left(I, \Sigma\left(S_{0}\right)\right), \boldsymbol{W} \in \mathscr{C}^{\infty}\left(I \times \partial S_{0}, \mathfrak{T}\left(\mathbb{R}^{+}\right)\right)$and $\boldsymbol{\Phi} \in \mathscr{C}^{\infty}\left(\partial S_{0}, \mathfrak{H}\left(\Sigma^{+}\right)\right)$be generic non zero terms independent of $\varepsilon$ corresponding to the three types of terms present in the expansion of $\boldsymbol{w}^{\varepsilon}$. Then we have

$$
\begin{aligned}
\|\boldsymbol{v}\|_{\mathrm{L}^{2}\left(\Omega^{\varepsilon}\right)} & =\mathcal{O}\left(\varepsilon^{1 / 2}\right), \quad\left\|\partial_{\alpha} \boldsymbol{v}\right\|_{\mathrm{L}^{2}\left(\Omega^{\varepsilon}\right)}=\mathcal{O}\left(\varepsilon^{1 / 2}\right), \quad \text { and } \quad\left\|\partial_{3} \boldsymbol{v}\right\|_{\mathrm{L}^{2}\left(\Omega^{\varepsilon}\right)}=\mathcal{O}\left(\varepsilon^{-1 / 2}\right), \\
\|\boldsymbol{W}\|_{\mathrm{L}^{2}\left(\Omega^{\varepsilon}\right)} & =\mathcal{O}\left(\varepsilon^{3 / 4}\right), \quad\left\|\partial_{\alpha} \boldsymbol{W}\right\|_{\mathrm{L}^{2}\left(\Omega^{\varepsilon}\right)}=\mathcal{O}\left(\varepsilon^{1 / 4}\right), \quad \text { and } \quad\left\|\partial_{3} \boldsymbol{W}\right\|_{\mathrm{L}^{2}\left(\Omega^{\varepsilon}\right)}=\mathcal{O}\left(\varepsilon^{-1 / 4}\right),
\end{aligned}
$$

and

$$
\|\Phi\|_{L^{2}\left(\Omega^{\varepsilon}\right)}=\mathcal{O}(\varepsilon), \quad \text { and } \quad\left\|\partial_{i} \Phi\right\|_{L^{2}\left(\Omega^{\varepsilon}\right)}=\mathcal{O}(1)
$$

Using these two lemmas, Proposition 7.2 and Proposition 3.2 of [15] giving the expansions of the operators $\tilde{e}_{i j}$, it is not hard to prove that

$$
\left\|\tilde{e}_{\alpha \beta}\left(\boldsymbol{v}^{0}+\varepsilon^{1 / 2} \boldsymbol{v}^{1 / 2}+\varepsilon \boldsymbol{v}^{1}\right)\right\|_{L^{2}\left(\Omega^{\varepsilon}\right)}=\mathcal{O}\left(\varepsilon^{1 / 2}\right),
$$

where we use the fact that $\zeta^{0} \neq 0$. Moreover, for all $k \geq 3$,

$$
\left\|\tilde{e}_{\alpha \beta}\left(\varepsilon^{k / 2} \boldsymbol{v}^{k / 2}\right)\right\|_{L^{2}\left(\Omega^{\varepsilon}\right)} \leq \mathcal{O}\left(\varepsilon^{(k+1) / 2}\right) .
$$

Similarly, writing the expansion of $\tilde{e}_{i j}$ in coordinates $\left(T, s, X_{3}\right)$ yields

$$
\left\|\tilde{e}_{\alpha \beta}\left(\boldsymbol{W}^{0}+\varepsilon^{1 / 2} \boldsymbol{W}^{1 / 2}+\varepsilon \boldsymbol{W}^{1}\right)\right\|_{L^{2}\left(\Omega^{\varepsilon}\right)} \leq \mathcal{O}\left(\varepsilon^{3 / 4}\right),
$$

and for all $k \geq 3$,

$$
\left\|\tilde{e}_{\alpha \beta}\left(\varepsilon^{k / 2} \boldsymbol{W}^{k / 2}\right)\right\|_{L^{2}\left(\Omega^{\varepsilon}\right)} \leq \mathcal{O}\left(\varepsilon^{(2 k+1) / 4}\right) .
$$

Moreover, we have for all $k \geq 2$

$$
\left\|\tilde{e}_{\alpha \beta}\left(\varepsilon^{k / 2} \boldsymbol{\Phi}^{k / 2}\right)\right\|_{L^{2}\left(\Omega^{\varepsilon}\right)} \leq \mathcal{O}\left(\varepsilon^{k / 2}\right) .
$$


Using the Kirchhoff-Love structure of the first terms of the formal series $\mathrm{V}[\varepsilon]$, we see (see [14] for further details) that under the condition $\zeta^{0} \neq 0$,

$$
\left\|\tilde{e}_{\alpha 3}\left(\boldsymbol{v}^{0}+\varepsilon^{1 / 2} \boldsymbol{v}^{1 / 2}+\varepsilon \boldsymbol{v}^{1}+\varepsilon^{3 / 2} \boldsymbol{v}^{3 / 2}\right)\right\|_{L^{2}\left(\Omega^{\varepsilon}\right)}=\mathcal{O}\left(\varepsilon^{3 / 2}\right),
$$

while for all $k \geq 4$,

$$
\left\|\tilde{e}_{\alpha 3}\left(\varepsilon^{k / 2} \boldsymbol{v}^{k / 2}\right)\right\|_{\mathrm{L}^{2}\left(\Omega^{\varepsilon}\right)} \leq \mathcal{O}\left(\varepsilon^{(k-1) / 2}\right) .
$$

Similarly, we compute that

$$
\left\|\tilde{e}_{\alpha 3}\left(\boldsymbol{W}^{0}+\varepsilon^{1 / 2} \boldsymbol{W}^{1 / 2}+\varepsilon \boldsymbol{W}^{1}\right)\right\|_{L^{2}\left(\Omega^{\varepsilon}\right)} \leq \mathcal{O}\left(\varepsilon^{5 / 4}\right),
$$

and for all $k \geq 3$

$$
\left\|\tilde{e}_{\alpha 3}\left(\varepsilon^{k / 2} \boldsymbol{W}^{k / 2}\right)\right\|_{\mathrm{L}^{2}\left(\Omega^{\varepsilon}\right)} \leq \mathcal{O}\left(\varepsilon^{(2 k-1) / 4}\right) .
$$

For the 3D boundary layer terms, we get for all $k \geq 2$,

$$
\left\|\tilde{e}_{\alpha 3}\left(\varepsilon^{k / 2} \boldsymbol{\Phi}^{k / 2}\right)\right\|_{\mathrm{L}^{2}\left(\Omega^{\varepsilon}\right)} \leq \mathcal{O}\left(\varepsilon^{k / 2}\right) .
$$

Finally, for the transverse strain, we obtain the estimates

$$
\left\|\tilde{e}_{33}\left(\boldsymbol{v}^{0}+\varepsilon^{1 / 2} \boldsymbol{v}^{1 / 2}+\varepsilon \boldsymbol{v}^{1}\right)\right\|_{L^{2}\left(\Omega^{\varepsilon}\right)}=\mathcal{O}\left(\varepsilon^{1 / 2}\right)
$$

and for all $k \geq 3,\left\|\tilde{e}_{33}\left(\varepsilon^{k / 2} \boldsymbol{v}^{k / 2}\right)\right\|_{\mathrm{L}^{2}\left(\Omega^{\varepsilon}\right)} \leq \mathcal{O}\left(\varepsilon^{(k-1) / 2}\right)$. Similarly, we have

$$
\left\|\tilde{e}_{33}\left(\boldsymbol{W}^{0}+\varepsilon^{1 / 2} \boldsymbol{W}^{1 / 2}+\varepsilon \boldsymbol{W}^{1}\right)\right\|_{L^{2}\left(\Omega^{\varepsilon}\right)} \leq \mathcal{O}\left(\varepsilon^{3 / 4}\right)
$$

and for $k \geq 2$,

$$
\left\|\tilde{e}_{33}\left(\varepsilon^{k / 2} \boldsymbol{W}^{k / 2}\right)\right\|_{L^{2}\left(\Omega^{\varepsilon}\right)} \leq \mathcal{O}\left(\varepsilon^{(2 k-1) / 4}\right) \quad \text { and } \quad\left\|\tilde{e}_{33}\left(\varepsilon^{k / 2} \boldsymbol{\Phi}^{k / 2}\right)\right\|_{L^{2}\left(\Omega^{\varepsilon}\right)} \leq \mathcal{O}\left(\varepsilon^{k / 2}\right) .
$$

Grouping together the previous estimates and using Theorem 8.2 for a sufficiently large $N$, we see that

$$
\left\|\tilde{e}_{\alpha \beta}\left(\boldsymbol{w}^{\varepsilon}\right)\right\|_{\mathrm{L}^{2}\left(\Omega^{\varepsilon}\right)}=\mathcal{O}\left(\varepsilon^{1 / 2}\right),
$$

where the main contribution comes from the first term $\zeta^{0} \neq 0$. Similarly,

$$
\left\|\tilde{e}_{\alpha 3}\left(\boldsymbol{w}^{\varepsilon}\right)\right\|_{L^{2}\left(\Omega^{\varepsilon}\right)} \leq \mathcal{O}(\varepsilon)
$$

where the main contribution comes from the first 3D boundary layer term $\Phi^{1}$ (this term may vanish even if $\zeta^{0} \neq 0$, as it depends only on traces of $\zeta^{0}$ : see the equation (5.13) of [15]). Eventually,

$$
\left\|\tilde{e}_{33}\left(\boldsymbol{w}^{\varepsilon}\right)\right\|_{\mathrm{L}^{2}\left(\Omega^{\varepsilon}\right)}=\mathcal{O}\left(\varepsilon^{1 / 2}\right)
$$

where the main contribution comes from $\zeta^{0}$. These estimates show the result. 
2. We prove now the second estimate in (1.20). Let $\mathrm{W} z$ be the operator corresponding to $\mathrm{U} \boldsymbol{z}$ through the shifter, that is $\mathrm{W} \boldsymbol{z}=\boldsymbol{\mu}^{-1}\left(x_{3}\right) \mathrm{U} \boldsymbol{z}$. We now prove that

$$
\tilde{E}_{3 \mathrm{D}}\left[\boldsymbol{w}^{\varepsilon}-\mathbf{W} \boldsymbol{z}^{\varepsilon}\right] \leq C \varepsilon \tilde{E}_{3 \mathrm{D}}\left[\boldsymbol{w}^{\varepsilon}\right]
$$

and this shows the result.

Recall that with $\boldsymbol{z}^{\varepsilon}$ we associate two formal series $\boldsymbol{\zeta}^{\prime}\left[\varepsilon^{1 / 2}\right]$ and $\boldsymbol{Z}^{\prime}[\varepsilon]$ given by Theorem 4.1. Using Proposition 7.2, it is easy to prove that we have an asymptotic expansion

$$
\boldsymbol{w}^{\varepsilon}-\mathbf{W} \boldsymbol{z}^{\varepsilon} \simeq \sum_{k \geq 0} \varepsilon^{k / 2}\left(\boldsymbol{e}^{k / 2}+\chi\left(\boldsymbol{E}^{k / 2}+\boldsymbol{\Phi}^{k / 2}\right)\right),
$$

where we have

$$
\boldsymbol{e}^{0}=\boldsymbol{e}^{1 / 2}=0 \quad \text { and } \quad \boldsymbol{e}^{1}=\overline{\boldsymbol{\zeta}}^{1},
$$

where $\bar{\zeta}^{1}=\boldsymbol{\zeta}^{1}-\boldsymbol{\zeta}^{\prime 1}$, and similarly

$$
\boldsymbol{E}^{0}=0, \quad \boldsymbol{E}^{1 / 2}=\left(\begin{array}{c}
0 \\
0 \\
\bar{Z}_{3}^{1 / 2}
\end{array}\right) \quad \text { and } \quad \boldsymbol{E}^{1}=\left(\begin{array}{l}
\bar{Z}_{T}^{1}-x_{3} \partial_{T} \bar{Z}_{3}^{1 / 2} \\
\bar{Z}_{s}^{1} \\
\bar{Z}_{3}^{1}
\end{array}\right)
$$

where $\bar{Z}_{3}^{k / 2}=Z_{3}^{k / 2}-Z_{3}^{\prime k / 2}$ for $k=1$ and 2 . As before, we compute that

$$
\left\|\tilde{e}_{\alpha \beta}\left(\boldsymbol{e}^{0}+\varepsilon^{1 / 2} \boldsymbol{e}^{1 / 2}+\varepsilon \boldsymbol{e}^{1}\right)\right\|_{L^{2}\left(\Omega^{\varepsilon}\right)} \leq \mathcal{O}\left(\varepsilon^{3 / 2}\right)
$$

and

$$
\left\|\tilde{e}_{\alpha \beta}\left(\boldsymbol{E}^{0}+\varepsilon^{1 / 2} \boldsymbol{E}^{1 / 2}+\varepsilon \boldsymbol{E}^{1}\right)\right\|_{L^{2}\left(\Omega^{\varepsilon}\right)} \leq \mathcal{O}\left(\varepsilon^{5 / 4}\right) .
$$

Moreover we have

$$
\left\|\tilde{e}_{\alpha 3}\left(\boldsymbol{e}^{0}+\varepsilon^{1 / 2} \boldsymbol{e}^{1 / 2}+\varepsilon \boldsymbol{e}^{1}\right)\right\|_{\mathrm{L}^{2}\left(\Omega^{\varepsilon}\right)} \leq \mathcal{O}\left(\varepsilon^{5 / 2}\right)
$$

and

$$
\left\|\tilde{e}_{\alpha 3}\left(\boldsymbol{E}^{0}+\varepsilon^{1 / 2} \boldsymbol{E}^{1 / 2}+\varepsilon \boldsymbol{E}^{1}\right)\right\|_{L^{2}\left(\Omega^{\varepsilon}\right)} \leq \mathcal{O}\left(\varepsilon^{7 / 4}\right) .
$$

Eventually, we have

$$
\tilde{e}_{33}\left(\boldsymbol{e}^{0}+\varepsilon^{1 / 2} \boldsymbol{e}^{1 / 2}+\varepsilon \boldsymbol{e}^{1}\right)=0 \quad \text { and } \quad \tilde{e}_{33}\left(\boldsymbol{E}^{0}+\varepsilon^{1 / 2} \boldsymbol{E}^{1 / 2}+\varepsilon \boldsymbol{E}^{1}\right)=0 .
$$

We conclude as before using Theorem 8.2 that

$$
\tilde{\mathrm{E}}_{3 \mathrm{D}}^{\varepsilon}\left[\boldsymbol{w}^{\varepsilon}-\mathbf{W} \boldsymbol{z}^{\varepsilon}\right] \leq \mathcal{O}\left(\varepsilon^{2}\right)=\mathcal{O}\left(\varepsilon \tilde{\mathrm{E}}_{3 \mathrm{D}}^{\varepsilon}\left[\boldsymbol{w}^{\varepsilon}\right]\right),
$$

where the mean contribution comes from the first 3D boundary layer term $\Phi^{1}$. This shows the Theorem.

\section{References}


[1] S. Agmon, A. Douglis, L. Nirenberg. Estimates near the boundary for solutions of elliptic partial differential equations satisfying general boundary conditions II. Comm. Pure Appl. Math. 17 (1964) 35-92.

[2] M. Bernadou, P. G. Ciarlet. Sur l'ellipticité du modèle linéaire de coques de W.T.Koiter. In R.Glowins Ki, J.L.Lions, editors, Computing Methods in Applied Sciences and Engineering, Lecture Notes in Economics and Mathematical Systems, Vol.134, pages 89-136. Springer-Verlag, Heidelberg 1976.

[3] B. Budiansky, J. L. Sanders. On the "best" first-order linear shell theory. In W. Prager Anniversary Volume, Progress in Applied Mechanics, pages 129-140. Macmillan, New-York 1967.

[4] P. G. Ciarlet. Mathematical elasticity. Vol. III. North-Holland Publishing Co., Amsterdam 2000. Theory of shells.

[5] P. G. Ciarlet, V. Lods. Asymptotic analysis of linearly elastic shells. I. Justification of membrane shell equations. Arch. Rational Mech. Anal. 136 (1996) 119-161.

[6] P. G. Ciarlet, V. Lods. Asymptotic analysis of linearly elastic shells. III. Justification of Koiter's shell equations. Arch. Rational Mech. Anal. 136 (1996) 191-200.

[7] P. G. Ciarlet, V. Lods, B. Miara. Asymptotic analysis of linearly elastic shells. II. Justification of flexural shell equations. Arch. Rational Mech. Anal. 136 (1996) 163-190.

[8] M. Dauge, , I. Djurdjevic, E. Faou, A. Rössle. Eigenmodes asymptotic in thin elastic plates. J. Maths. Pures Appl. 78 (1999) 925-964.

[9] M. Dauge, E. Faou. Koiter estimate revisited. Preprint (2001).

[10] M. Dauge, I. Gruais. Asymptotics of arbitrary order for a thin elastic clamped plate. I: Optimal error estimates. Asymptotic Analysis 13 (1996) 167-197.

[11] M. Dauge, I. Gruais. Asymptotics of arbitrary order for a thin elastic clamped plate. II: Analysis of the boundary layer terms. Asymptotic Analysis 16 (1998) 99124.

[12] M. Dauge, I. Gruais, A. Rössle. The influence of lateral boundary conditions on the asymptotics in thin elastic plates. SIAM J. Math. Anal. 31(2) (1999/00) 305345 (electronic).

[13] G. Duvaut, J.-L. Lions. Les Inéquations en Mécanique et en Physique. Dunod, Paris 1972.

[14] E. FAOU. Développements asymptotiques dans les coques linéairement élastiques. Thèse, Université de Rennes 1 (2000).

[15] E. FAOU. Elasticity on a thin shell: Formal series solution. Asymptotic Analysis 31 (2002) 317-361.

[16] K. Genevey. A regularity result for a linear membrane shell problem. RAIRO Modél. Math. Anal. Numér. 30 (4) (1996) 467-488. 
[17] R. D. GRegory, F. Y. WAN. Decaying states of plane strain in a semi-infinite strip and boundary conditions for plate theory. J. Elasticity 14 (1984) 27-64.

[18] F. John. Refined interior equations for thin elastic shells. Comm. Pure Appl. Math. 24 (1971) 583-615.

[19] W. T. KoITER. A consistent first approximation in the general theory of thin elastic shells. Proc. IUTAM Symposium on the Theory on Thin Elastic Shells, August 1959 (1960) 12-32.

[20] W. T. Koiter. On the foundations of the linear theory of thin elastic shells: I. Proc. Kon. Ned. Akad. Wetensch., Ser.B 73 (1970) 169-182.

[21] W. T. KoITER. On the foundations of the linear theory of thin elastic shells: II. Proc. Kon. Ned. Akad. Wetensch., Ser.B 73 (1970) 183-195.

[22] V. Lods, C. MARDARE. Asymptotic justification of the Kirchhoff-love hypotheses for a linearly elastic clamped shell. J. Elasticity 58 (2000) 105-154.

[23] V. Lods, C. Mardare. Justification of linear Koiter and Naghdi's models for totally clamped shell. Asymptotic Anal. 31 (2002) 189-210.

[24] C. MARDARE. Asymptotic analysis of linearly elastic shells: error estimates in the membrane case. Asymptot. Anal. 17 (1998) 31-51.

[25] P. M. NAGHDi. Foundations of elastic shell theory. In Progress in Solid Mechanics, volume 4, pages 1-90. North-Holland, Amsterdam 1963.

[26] S. A. NAZARov, I. S. Zorin. Edge effect in the bending of a thin threedimensional plate. Prikl. Matem. Mekhan. 53 (4) (1989) 642-650. English translation J. Appl. Maths. Mechs. (1989) 500-507.

[27] V. V. NovozhiLov. Thin Shell Theory. Walters-Noordhoff Publishing, Groningen 1959.

[28] J. Pitkëranta, A.-M. Matache, C. Schwab. Fourier mode analysis of layers in shallow shell deformations. Comput. Methods Appl. Mech. Eng. 190 (2001) 2943-2975.

[29] J. Sanchez-Hubert, E. Sanchez-Palencia. Coques élastiques minces. Propriétés asymptotiques. Recherches en mathématiques appliquées. Masson, Paris 1997.

[30] M. I. VishiK, L. A. LYUSTERNIK. Regular degeneration and boundary layers for linear differential equations with small parameter. Amer. Math. Soc. Transl. (2) 20 (1962) 239-364.

\section{Author's address}

Erwan FAOU

INRIA Rennes, Campus de Beaulieu, 35042 Rennes, FRANCE

Erwan.Faoudirisa.fr 